\title{
Polynomial approximation in several variables *
}

\author{
Vilmos Totik ${ }^{\dagger}$ \\ Dedicated to Zeev Ditzian
}

\begin{abstract}
This paper is about the characterization of the rate of best polynomial approximation on a domain in $\mathbf{R}^{d}$ by multivariate polynomials of degree $n$ via a suitable modulus of continuity or smoothness. We shall be concerned with the situations: approximation on convex polytopes and approximation on (not necessarily convex) smooth algebraic domains. We shall introduce appropriate moduli of smoothness and prove the corresponding Jackson theorem. The usual weak converses are also true.
\end{abstract}

\section{Contents}

I Approximation on polytopes 2

1 The main result $\quad 2$

2 Outline of the proof of Theorem $1.1 \quad 5$

3 Pyramidal covering and approximation on general polytopes 6

3.1 Lemmas on geometry and polynomial partitions . . . . . . . . . 7

3.2 Pyramidal coverings . . . . . . . . . . . . . . . 12

List of notations used in the pyramidal covering construction . . 15

3.3 Patching approximants on a pyramidal covering together to a global approximant . . . . . . . . . . . . . . 16

4 Approximation on pyramids and the proof of Theorem 1.1 for large degrees

5 Small degrees

II Approximation on algebraic domains

*AMS Classification: 41A10, 41A17 Keywords: polynomials, several variables, approximation, moduli of smoothness, polytopes, algebraic domains

†Supported by ERC Advanced Grant No. 267055 and by NSF DMS 1564541 
6 Domains and moduli of smoothness 28

6.1 Domains in $\mathbf{R}^{2} \ldots \ldots \ldots \ldots \ldots \ldots \ldots \ldots$

6.2 Domains in $\mathbf{R}^{d} \ldots \ldots \ldots \ldots \ldots \ldots \ldots$

6.3 The Jackson theorem and its converse . . . . . . . . . . . 30

7 Reduction to approximation close to the boundary 32

$\begin{array}{lll}8 & \text { The } r=1 \text { case } & 38\end{array}$

9 Approximation on disks $\quad 41$

10 A detour on the derivative of composite functions 45

11 Proof of Theorem 6.5 48

One of the primary questions of approximation theory is to characterize the rate of approximation by a given system in terms of some (computable) modulus of continuity or smoothness. In this spirit we set out the task to characterize the rate of best polynomial approximation on a domain in $\mathbf{R}^{d}$ by multivariate polynomials of degree $n$ via a suitable modulus of continuity or smoothness.

We shall be concerned with the situations: approximation on convex polytopes and approximation on (not necessarily convex) smooth algebraic domains. We shall introduce appropriate moduli of smoothness and prove the corresponding Jackson theorem. The usual weak converses are also true, they actually automatically follow from classical converse results and from the way the moduli of smoothness are defined.

\section{Part I}

\section{Approximation on polytopes}

In this first part we shall be concerned with convex polytopes in any dimension.

\section{The main result}

Let $f$ be a continuous function on $[-1,1]$. With $\varphi(x)=\sqrt{1-x^{2}}$ and $r=1,2, \ldots$ let

$$
\omega_{\varphi}^{r}(f, \delta)=\sup _{0<h \leq \delta, x \in[-1,1]}\left|\Delta_{h \varphi(x)}^{r} f(x)\right|
$$

be its so called $\varphi$-modulus of smoothness of order $r$, where

$$
\Delta_{h}^{r} f(x)=\sum_{k=0}^{r}(-1)^{k}\left(\begin{array}{l}
r \\
k
\end{array}\right) f\left(x+\left(\frac{r}{2}-k\right) h\right)
$$


is the $r$-th symmetric difference. In (1.1) it is agreed that $\Delta_{h}^{r} f(x)=0$ if $[x-$ $\left.\frac{r}{2} h, x+\frac{r}{2} h\right] \nsubseteq[-1,1]$. See the book [6] for this kind of moduli of smoothness, for their properties and for their applications.

With this modulus of smoothness one can characterize the rate of best polynomial approximation on $[-1,1]$. Indeed, if

$$
E_{n}(f)=\inf _{P_{n}}\left\|f-P_{n}\right\|_{[-1,1]}
$$

is the error (in the supremum norm) of the best polynomial approximation by polynomials of degree at most $n$, then the Jackson-type estimate

$$
E_{n}(f) \leq C_{r} \omega_{\varphi}^{r}(f, 1 / n),
$$

as well as its weak converse

$$
\omega_{\varphi}^{r}(f, 1 / n) \leq C_{r} \frac{1}{n^{r}} \sum_{k=0}^{n}(k+1)^{r-1} E_{k}(f)
$$

are true (see [6, Theorem 7.2.1] and [6, Theorem 7.2.4]). Our aim in this paper is to find the analogues of these in several variables.

In $\mathbf{R}^{d}$ we call a closed set $K \subset \mathbf{R}^{d}$ a convex polytope if it is the convex hull of finitely many points. $K$ is $d$-dimensional if it has an inner point, which we shall always assume. The analogue of the $\varphi$-modulus of smoothness on $K$ was defined in [6, Chapter 12], and to recall its definition we need to consider the given function along lines in different directions. A direction $\underline{e}$ in $\mathbf{R}^{d}$ is just a unit vector $\underline{e} \in \mathbf{R}^{d}$. Clearly, $\underline{e}$ can be identified with an element of the unit sphere $S^{d-1}$, so $S^{d-1}$ is the set of all directions in $\mathbf{R}^{d}$. Let $K$ be a convex polytope, $x \in K$ and $\underline{e} \in S^{d-1}$ a direction. The line $l_{e, x}$ through $x$ which is parallel with $\underline{e}$ intersects $K$ in a segment $A_{\underline{e}, x} B_{\underline{e}, x}$. The role of $\varphi$ on that segment is taken over by

$$
\tilde{d}_{K}(\underline{e}, x)=\sqrt{\operatorname{dist}\left(x, A_{\underline{e}, x}\right) \cdot \operatorname{dist}\left(x, B_{\underline{e}, x}\right)} .
$$

If $f$ is a continuous function on $K$, then we define its $r$-th symmetric differences in the direction of $\underline{e}$ as

$$
\Delta_{h \underline{e}}^{r} f(x)=\sum_{k=0}^{r}(-1)^{k}\left(\begin{array}{l}
r \\
k
\end{array}\right) f\left(x+\left(\frac{r}{2}-k\right) h \underline{e}\right)
$$

with the agreement that this is 0 if $x+\frac{r}{2} h \underline{e}$ or $x-\frac{r}{2} h \underline{e}$ does not belong to $K$.

If $\mathcal{E}$ is a set of directions, then the $r$-th modulus of smoothness with these directions is defined as (see [6, Section 12.2])

$$
\omega_{K}^{r}(f, \delta)_{\mathcal{E}}=\sup _{e \in \mathcal{E}, h \leq \delta, x \in K}\left|\Delta_{h \tilde{d}_{K}(\underline{e}, x) \underline{e}}^{r} f(x)\right| .
$$

If $\mathcal{E}$ is the direction of the edges of $K$, then we shall simply write $\omega_{K}^{r}(f, \delta)$ for $\omega_{K}^{r}(f, \delta)_{\mathcal{E}}$. These moduli of smoothness share many properties of classical moduli, in particular

$$
\omega_{K}^{r}(f, \lambda \delta)_{\mathcal{E}} \leq C_{r} \lambda^{r} \omega_{K}^{r}(f, \delta)_{\mathcal{E}}
$$


for any $\lambda \geq 1$. We shall also frequently use monotonicity in $K$ : if $K_{1} \subset K_{2}$ then $\omega_{K_{1}}^{r}(f, \delta)_{\mathcal{E}} \leq \omega_{K_{2}}^{r}(f, \delta)_{\mathcal{E}}$, which is immediate from the definitions.

Note that when $K=[-1,1]$, then there is only one direction (and its negative) and this modulus of smoothness takes the form (1.1), i.e.

$$
\omega_{\varphi}^{r}(f, \delta)=\omega_{[-1,1]}^{r}(f, \delta) .
$$

Let $\Pi_{n}^{d}$ be the set of polynomials in $d$-variables of total degree at most $n$, and let the supremum norm on a set $K$ be denoted by $\|\cdot\|_{K}$. For a continuous function $f \in C(K)$ we are interested in

$$
E_{n}(f)_{K}=\inf _{P_{n} \in \Pi_{n}^{d}}\left\|f-P_{n}\right\|_{K},
$$

which is the error of best approximation of $f$ by polynomials of total degree at most $n$.

In the first part of this paper we prove

Theorem 1.1 If $K$ is a convex polytope in $\mathbf{R}^{d}$, then for $r=1,2, \ldots$

$$
E_{n}(f)_{K} \leq C \omega_{K}^{r}(f, 1 / n), \quad n \geq r d .
$$

Here the constant $C$ depends only on $K$ and $r$.

Call a polytope $K$ in $\mathbf{R}^{d}$ a simple polytope if at every vertex there are precisely $d$ edges. Theorem 1.1 was verified in [6, Theorem 12.1.1] for cubes/parallelepipeds in all dimensions, and in [6, Theorem 12.2.3] (with an additional term) for all simple polytopes. The somewhat weaker result

$$
E_{n}(f)_{K} \leq C \omega_{K}^{r}(f, 1 / n)_{S^{d-1}},
$$

i.e. when all directions are used in the modulus of smoothness, was proven in [14, Chapters 1-10] for all polytopes in $R^{3}$. In [14, Chapter 11, Theorem 1.1], it was also stated that (1.10) holds also in $\mathbf{R}^{d}, d>3$, but Andriy Prymak noticed that the proof (that reduced the problem to approximation on simple polytopes) does not give that, so the claim was wrong in the sense that the proof in [14, Chapter 11] yields (1.10) only for special polytopes $K$ (which satisfy the property that the intersection of $K$ with any hyperplane that cuts off a vertex is a simple $(d-1)$-dimensional polytope). Thus, in its generality, (1.9) (which also covers (1.10)) is new (and actually sharper than what has been previously proven in the special cases known in the literature).

The weak converse

$$
\omega_{K}^{r}(f, 1 / n) \leq C_{r} \frac{1}{n^{r}} \sum_{k=0}^{n}(k+1)^{r-1} E_{k}(f)_{K},
$$

is an immediate consequence of (1.3) if we take into account how the modulus of smoothness on the left has been defined.

The Jackson-type estimate Theorem 1.1 and the weak converse (1.3) easily imply the following. If $K_{1} \subset K$ is a segment and we consider $f$ only on $K_{1}$, then 
we obtain a continuous function on a segment/interval, and we can consider the approximation of this one-variable function by polynomials of the same (single) variable. Now it follows that if $\alpha>0$ and the error of best approximation of $f$ on any segment of $K$ by (one variable) polynomials of degree at most $n$ is $\leq n^{-\alpha}$, then on the whole $K$ the function $f$ can be approximated with error $\leq C n^{-\alpha}$ by polynomials (of several variables) of degree at most $n$ (where $C$ depends only on $K$ ).

Remark 1.2 We need to say a few words on the degree of polynomials. In this work we use total degree (i.e. the degree of $x_{1}^{\alpha_{1}} \cdots x_{d}^{\alpha_{d}}$ is $\alpha_{1}+\cdots+\alpha_{d}$ ), while the works [6] and [14] used maximal degree in the separate variables (in which case the degree of $x_{1}^{\alpha_{1}} \cdots x_{d}^{\alpha_{d}}$ is $\max \alpha_{j}$ ). This has not much effect on the corresponding Jackson theorem, only on the range of $n$ for which the theorem holds. Indeed, let $E_{n}(f)$ be as in (1.8) where the infimum is taken for all polynomials of total degree at most $n$, and let

$$
E_{n}^{*}(f)_{K}=\inf _{Q_{n}}\left\|f-Q_{n}\right\|_{K},
$$

where now the infimum is taken for all polynomials $Q_{n}$ that are of degree at most $n$ in each variable. It is clear that $E_{n d}(f)_{K} \leq E_{n}^{*}(f)_{K} \leq E_{n}(f)_{K}$, so a Jackson-type estimate for either of $E_{n}$ or $E_{n}^{*}$ yields a Jackson-type estimate for the other. Note however, that if $f_{0}$ is the polynomial $x_{1}^{r-1} \cdots x_{d}^{r-1}$ and $K$ is the unit cube in $\mathbf{R}^{d}$, then $E_{n}^{*}\left(f_{0}\right)=0$ for $n \geq r-1$ while $E_{n}\left(f_{0}\right)=0$ only for $n \geq d(r-1)$. In a similar manner, if we use edge directions in forming the modulus of smoothness, then $\omega^{r}\left(f_{0}, \delta\right)_{K} \equiv 0$, so when using $E_{n}(f)$ the Jacksontype estimate (1.9) cannot hold for $n<d(r-1)$. That is why we claimed (1.9) only for $n \geq r d$.

\section{Outline of the proof of Theorem 1.1}

In the proof of Theorem 1.1 a special role is played by pyramids. Let $S_{0}$ be a convex $(d-1)$-dimensional polytope in $\mathbf{R}^{d}$, and $P \in \mathbf{R}^{d}$ a point outside the hyperplane of $S_{0}$. The convex hull of $S_{0}$ and $P$ determines a $d$-dimensional pyramid $S$ with apex at $P$. We call $S_{0}$ the base of $S$, and the edges emanating from $P$ the apex edges of $S$.

Let $K \subset \mathbf{R}^{d}$ be a convex $d$-dimensional polytope with vertices $\left\{Q_{j}\right\}_{j=1}^{m}$. If $Q_{j}$ is a vertex of $K$ and $L$ is a hyperplane that intersects all the edges emanating from $Q_{j}$, then $L$ cuts off a pyramid $S_{j}$ from $K$ with apex at $Q_{j}$ (and with base $K \cap L)$.

As has been mentioned, (1.10) was proven in [14] in $\mathbf{R}^{3}$. That proof works in any dimension for a polytope $K$ which has the property that if we cut off a small pyramid $S_{j}$ around every vertex $Q_{j}$, then what remains (say $K_{0}$ ) is a simple polytope (note that in $\mathbf{R}^{2}$ any polytope is simple, so the just mentioned property is true for any 3 -dimensional polytopes). In that approach simple polytopes are used in two essential ways. 
- 1) $K_{0}$ is simple, so for it the claim (say (1.10)) on the rate of approximation is true by [6, Theorem 12.2.3] (with the additional term in that theorem separately removed).

- 2) If $S_{j}^{\lambda}$ is the $\lambda$-shrunk copy of $S_{j}$ from $Q_{j}$, then the truncated pyramid $S_{j} \backslash S_{j}^{\lambda}$ is simple, so for it the claim on the rate of approximation is true again by [6, Theorem 12.2.3].

Note that if we are working in $\mathbf{R}^{3}$, then no assumption is needed, $K_{0}$ is always simple, so the just outlined approach yields the theorem. This is no longer true in $\mathbf{R}^{d}, d>3$ (an observation by A. Prymak ${ }^{1}$ ), so the use of simple polytopes in 1)-2) must be avoided. The proof we give is by induction on the dimension, but for the induction we need the approximants to be given by linear operators, and the modulus of smoothness should involve only edge-directions.

A) In 2) the truncated pyramid is (for $\lambda<1$ close to 1 ) the union of welloverlapped product sets (with the smaller base of the truncated pyramid $\times$ the edges), so induction and simple patching gives the result for the truncated pyramid. From there the proof in [14] gives the result for each of the pyramids $S_{j}$.

B) The problem in 1) is avoided by not looking at all on the remaining polytope $K_{0}$, but rather appropriately covering the original polytope by translated copies of the pyramids $S_{j}$ and patching the approximants on them (which were established in A) together.

We start with the last tusk, i.e. by constructing an appropriate pyramidal covering.

\section{Pyramidal covering and approximation on gen- eral polytopes}

We shall write $a_{n} \prec b_{n}$ if there is a $C>0$ such that for sufficiently large $n$ we have $\left|a_{n}\right| \leq C b_{n}$. If we also specify a range $n \geq n_{0}$ for $n$, then $a_{n} \prec b_{n}, n \geq n_{0}$ means that there is a $C>0$ for which $\left|a_{n}\right| \leq C b_{n}$ for all $n \geq n_{0}$.

We shall prove Theorem 1.1 using bounded linear operators mapping $C(K)$ into $\Pi_{n}^{d}$ (since $\Pi_{n}^{d}$ is finite dimensional, it does not matter what norm is used on $\left.\Pi_{n}^{d}\right)$. Therefore, we introduce the notation

$$
E_{n}^{L}(f)_{K} \prec \omega(1 / n)
$$

to denote the fact that for all sufficiently large $n$ (independent of $f$ ) there are bounded linear operators $L_{n}: C(K) \rightarrow \Pi_{n}^{d}$ such that

$$
\left\|f-L_{n} f\right\|_{K} \leq C \omega(1 / n) .
$$

\footnotetext{
${ }^{1}$ Indeed, if $V$ is a non-simple polytope in $\mathbf{R}^{d-1}, d>3$, and $K$ is the convex hull in $\mathbf{R}^{d}$ of $V$ and the point $(0, \ldots, 0,1) \in \mathbf{R}^{d}$, then for any hyperplane $L$ in $\mathbf{R}^{d}$ that is parallel with the $x_{d}=0$ hyperplane that intersects $K$ in at least two points the intersection $K \cap L$ is similar to $V$, hence $L$ cuts off a non-simple pyramid from $K$.
} 
with some constant $C$ independent of $f$ and $n$. Sometimes we need to specify a subclass $\mathcal{C}$ of $C(K)$ for which (3.2) is required, in which case we write

$$
E_{n}^{L}(f)_{K} \prec \omega(1 / n), \quad f \in \mathcal{C} .
$$

(3.1) proves Theorem 1.1 for sufficiently large $n$. Small $n$ 's will be separately handled in Theorem 5.1 in Section 5.

In this section we show that if one can approximate in the correct order on pyramids, then the approximation on any polytope follows by a pyramidal covering of the polytope. Then, in the next section, we show how to handle pyramids.

We shall need some simple geometric facts.

\subsection{Lemmas on geometry and polynomial partitions}

Lemma 3.1 For every convex body $S \subset \mathbf{R}^{d}$ there is a constant $\alpha>0$ with the following property: if we represent $S$ as the intersection of half spaces, then for any point $P$ in $\mathbf{R}^{d}$ the distance from $P$ to one of these half spaces is at least $\alpha$ times the distance from $P$ to $S$.

Proof. Let us write a ball $B_{r}$ of some radius $r$ inside $S$ about a point $O$ of $S$, and let $R$ be such that the ball $B_{R}$ about $O$ of radius $R$ contains $S$ in its interior. We show that for any $\beta<1$ the number $\alpha=\beta r / R$ is suitable.

Indeed, let $P$ be any point outside $S$. The $O P$ segment intersects the boundary of $S$ at a point $Q$ (see Figure 1). Then $\overline{P Q} \geq \operatorname{dist}(P, S)$, so if we select the point $q$ on the segment $P Q$ close to $Q$, then $\overline{P q} \geq \beta \operatorname{dist}(P, S)$ and at the same time $\overline{O q}<R$ are satisfied (note that $Q$ lies inside $B_{R}$, so we may select $q$ so that it also lies inside that ball). There is a half space $K$ among the given half spaces that contains $S$ but does not contain the point $q$. Let $H$ be the boundary of that half space. Then $H$ intersects the $O P$ segment in a point $A$ (that lies necessarily on the segment $Q q$ ), and consider the normal line to $H$ at the point $A$. This normal line and the line passing through $O$ and $P$ determines a plane $L$ that intersects $H$ in a line $\ell$ (see below for the case when these two lines coincide). Let $B$ be the closest point of $\ell$ to $O$ and $C$ the closest point of $\ell$ to $P$. Since $L$ is perpendicular to $H$ and $P \in L$, the distance from $P$ to $C$ is precisely the distance from $P$ to the half space $K$. Furthermore, $\overline{O B} \geq r$ ( $K$ contains the ball $B_{r}$ ), $\overline{O A}<R$ ( $A$ lies on the segment $Q q$ which lies inside $\left.B_{R}\right)$, and from the similarity of the triangles $A C P$ and $A B O$ we obtain that $\overline{P C} / \overline{P A}=\overline{O B} / \overline{O A}>r / R$. Since $\overline{P A} \geq \overline{P q} \geq \beta \operatorname{dist}(P, S)$ is also true, we finally obtain

$$
\operatorname{dist}(P, K)=\overline{P C}>(r / R) \overline{P A} \geq(r / R) \beta \operatorname{dist}(P, S) .
$$

The just given proof used that the normal line to $H$ at $A$ and the line passing through $O$ and $P$ are different (so they form a plane). If they are the same, then $\operatorname{dist}(P, K)=\overline{P A} \geq \overline{P q} \geq \beta \operatorname{dist}(P, S)$. 


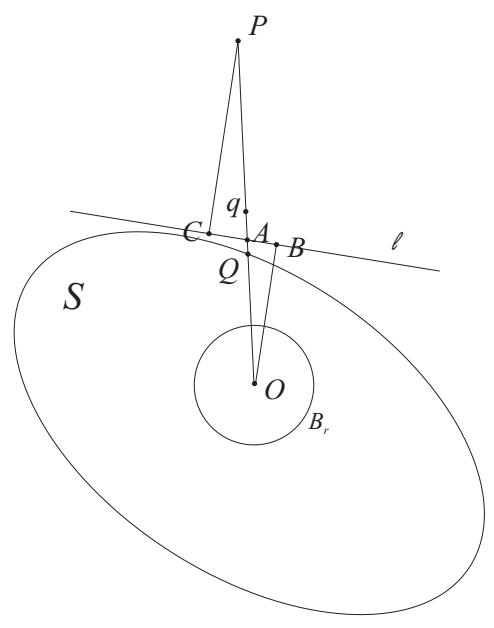

Figure 1:

Lemma 3.2 Let $S$ be a convex polytope lying in the unit ball $B_{1}(0)$ of $\mathbf{R}^{d}$, and let $\varepsilon>0$. There is a constant $k=k(S, \varepsilon)$ such that for every $n \geq 1$ there are polynomials $R_{k n}$ of degree at most kn for which

a) $0 \leq R_{k n}(x) \leq 1$ for $x \in B_{1}(0)$,

b) $1-R_{k n}(x) \leq 2^{-n}$ for $x \in S$,

c) $R_{k n}(x) \leq 2^{-n}$ for $x \in B_{1}(0)$, $\operatorname{dist}(x, S) \geq \varepsilon$.

Remark 3.3 1. The lemma is closely related to well localized polynomial partitions of unity. Indeed, if $S_{1}, \ldots, S_{m}$ are finitely many closed convex bodies in $B_{1}(0)$ and $U_{1}, \ldots, U_{m}$ are neighborhoods of them, then there is a constant $\theta>0$ and for each large $n$ there are polynomials $P_{1, n}, \ldots, P_{m, n}, P_{m+1, n}$ of degree at most $n$ such that

- $0 \leq P_{j, n} \leq 1$ on $B_{1}(0)$ for all $j$,

- $\sum_{j=1}^{m+1} P_{j, n} \equiv 1$,

- $P_{j, n} \leq \theta^{n}$ on $B_{1}(0) \backslash U_{j}$ for all $1 \leq j \leq m$ and

- $P_{m+1, n} \leq \theta^{n}$ on $\cup_{j=1}^{m} S_{j}$.

In particular, if the $S_{j}$ are pairwise disjoint, then (by choosing $U_{j}$ to lie outside $\cup_{s \neq j} S_{s}$ ) we can also have (with a possibly large $\theta$ )

- $1-\theta^{n} \leq P_{j} \leq 1$ on $S_{j}$ for all $1 \leq j \leq m$. 
Indeed, to see that, we may assume that each $S_{j}$ is a convex polytope (just place such a polytope in between $S_{j}$ and $U_{j}$ ). Then we can apply Lemma 3.2 to each $S_{j}$ (with an $\varepsilon$ for which the $\varepsilon$-neighborhood of $S_{j}$ is part of $U_{j}$ ) to get polynomials $R_{j, n / m}$ of degree at most $n / m$ as in Lemma 3.2 (just apply the lemma with $n$ replaced by $n / \mathrm{km}$ ). Now it is easy to see that

$$
P_{j, n}=R_{j, n / m}\left(1-R_{1, n / m}\right) \cdots\left(1-R_{j-1, n / m}\right), \quad j=1, \ldots, m
$$

and

$$
P_{m+1, n}=\left(1-R_{1, n / m}\right) \cdots\left(1-R_{m, n / m}\right)
$$

satisfy the requirements. The $\theta$ depends on $m, \varepsilon$ and on the numbers $k\left(S_{j}, \varepsilon\right)$ in Lemma 3.2.

2. To our knowledge in more than one variables polynomial partitions of unity were created for the fist time in [7] (that paper dealt with dimension 2). There the decrease was of power type away from the sets, and that decrease started right at the boundary of the sets $S_{j}$. In the just given construction $P_{j, n}$ tends to 0 geometrically fast outside $U_{j}$, though there is no information on its behavior in $U_{j} \backslash S_{j}$. In patching local polynomial approximants together to a global approximant one needs, in general, a geometric rate of convergence to 0 as just has been explained.

Proof of of Lemma 3.2. Let $\alpha>0$ be the constant from Lemma 3.1 for the polytope $S$.

It is well known (see e.g. [12, Corollary VI.3.6]) that for every $\varepsilon>0$ there are an $s$ and for every $n$ polynomials $\tilde{R}_{s n}\left(x_{1}\right)$ of a single variable $x_{1}$ of degree $s n$ such that $0 \leq \tilde{R}_{s n} \leq 1$ on $[-3,3], 1-\tilde{R}_{s n} \leq 3^{-n}$ on $[-3,0]$ and $\tilde{R}_{s n} \leq 3^{-n}$ on $[\alpha \varepsilon, 3]$. We regard $\tilde{R}_{s n}$ as a polynomial of degree $s n$ of the variables $\left(x_{1}, \ldots, x_{d}\right)$.

$S$ is the intersection of some half-spaces $K_{1}, \ldots, K_{m}$. We may assume that the boundary of each $K_{j}$ contains a point of $S$. If $\Phi_{j}$ is an isometry of $\mathbf{R}^{d}$ that maps $K_{j}$ into the half-space $x_{1} \leq 0$, then $\tilde{R}_{s n}\left(\Phi_{j}(x)\right)$ is a polynomial of degree at most $s n$, it is in between 0 and 1 on the unit ball (note that the unit ball is mapped into the strip $-3 \leq x_{1} \leq 3$ by $\Phi_{j}$ ), it is closer to 1 than $3^{-n}$ on $S$ ( $S$ is mapped into $-3 \leq x_{1} \leq 0$ ) and it is closer to 0 than $3^{-n}$ at any point of $B_{1}(0) \backslash K_{j}$ which lies of distance $\geq \alpha \varepsilon$ from $K_{j}$. However, by the choice of $\alpha$, any point in the unit ball that is of distance $\geq \varepsilon$ from $S$ lies of distance $\geq \alpha \varepsilon$ from one of these $K_{j}$, hence, the product of the polynomials $\tilde{R}_{s n}\left(\Phi_{j}(x)\right)$, $j=1, \ldots, m$, is appropriate in the lemma for sufficiently large $n$.

Lemma 3.4 Let $S \subset K$ be convex polytopes in $\mathbf{R}^{d}$, and let $S^{\varepsilon}$ be the closed $\varepsilon$-neighborhood of $S$. Let furthermore $H \subset K$ be a closed set such that for some $\delta>0$ the intersection $S \cap H$ contains a (d-dimensional) ball of radius $\delta$. Then for every $l_{0}$ there is an $l$ such that

$$
E_{l_{0} n}^{L}(f)_{S^{\varepsilon} \cap K} \prec \omega(1 / n), \quad E_{l_{0} n}^{L}(f)_{H} \prec \omega(1 / n), \quad f \in C(K)
$$


imply

$$
E_{l n}^{L}(f)_{S \cup H} \prec \omega(1 / n), \quad f \in C(K) .
$$

Here the constant in the last $\prec$ does not depend on $f$, it depends only on the diameter of $K$, on $\delta$ and on the constants in the preceding two $\prec$. The quantity $\omega(1 / n)$ is a generic term that tends to 0 as $n \rightarrow \infty$ - we shall use the lemma with $\omega(1 / n)$ replaced by the $1 / n$-value of a certain modulus of smoothness. Recall also our convention that the implication (3.4) $\Rightarrow(3.5)$ is not for individual functions, but should be read that if (3.4), i.e.

$$
E_{l_{0} n}^{L}(f)_{S^{\varepsilon} \cap K} \leq C_{0} \omega(1 / n), \quad E_{l_{0} n}^{L}(f)_{H} \leq C_{0} \omega(1 / n), \quad n \geq n_{0}
$$

holds for all functions $f \in C(K)$ with some constants $C_{0}, n_{0}$, then (3.5), i.e.

$$
E_{l n}^{L}(f)_{S \cup H} \leq C_{1} \omega(1 / n), \quad n \geq n_{1}
$$

is also true with some $C_{1}, n_{1}$ for all such $f$.

Proof. We may assume $K \subset B_{1}(0)$, i.e. that $K$ lies in the unit ball of $\mathbf{R}^{d}$.

First we recall

Lemma 3.5 [14, Lemma 4.1] If $B$ is a ball of radius $\delta$ lying in the unit ball $B_{1}(0)$, then for any polynomial $Q_{n}$ of degree at most $n$

$$
\left\|Q_{n}\right\|_{B_{1}(0)} \leq\left\|Q_{n}\right\|_{B}(4 / \delta)^{n} .
$$

Let $P_{1, n}$ and $P_{2, n}$ be bounded linear operators on $C(K)$ mapping into $\Pi_{l_{0} n}^{d}$ such that

$$
\left\|f-P_{1, n} f\right\|_{S^{\varepsilon} \cap K} \leq C \omega(1 / n), \quad\left\|f-P_{2, n} f\right\|_{H} \leq C \omega(1 / n)
$$

for $f \in C(K)$. By assumption there is a ball $B \subseteq H \cap S$ of some radius $\delta>0$, and on that ball we have

$$
\left\|P_{1, n} f-P_{2, n} f\right\|_{B} \leq\left\|f-P_{2, n} f\right\|_{H}+\left\|f-P_{1, n} f\right\|_{S^{\varepsilon} \cap K} \leq 2 C \omega(1 / n),
$$

hence, by Lemma 3.5

$$
\left\|P_{1, n} f-P_{2, n} f\right\|_{B_{1}(0)} \leq 2 C(4 / \delta)^{l_{0} n} \omega(1 / n) .
$$

Choose an integer $b$ such that $(1 / 2)^{b}<(\delta / 4)^{l_{0}}$ and with the polynomials $R_{k n}$ from Lemma 3.2 for $S$ and with $l=\left(l_{0}+k b\right)$ set

$$
L_{l n} f=R_{k b n} P_{1, n} f+\left(1-R_{k b n}\right) P_{2, n} f .
$$

This is a bounded linear operator from $C(K)$ to $\Pi_{l n}^{d}$, and for it we have

- on $H \cap S^{\varepsilon}$

$$
\left|f-L_{l n} f\right| \leq R_{k b n}\left|f-P_{1, n} f\right|+\left(1-R_{k b n}\right)\left|f-P_{2, n} f\right| \leq C \omega(1 / n),
$$


- on $S$ (see (3.7))

$$
\begin{aligned}
\left|f-L_{l n} f\right| & =\left|f-P_{1, n} f+\left(1-R_{k b n}\right)\left(P_{1, n} f-P_{2, n} f\right)\right| \leq\left|f-P_{1, n} f\right|+ \\
& +\left(1-R_{k b n}\right)\left|P_{1, n} f-P_{2, n} f\right| \leq C \omega(1 / n)+2^{-b n} 2 C(4 / \delta)^{l_{0} n} \omega(1 / n) \\
& \leq 3 C \omega(1 / n),
\end{aligned}
$$

- and on $H \backslash S^{\varepsilon}$

$$
\begin{aligned}
\left|f-L_{l n} f\right| & =\mid\left(f-P_{2, n} f-R_{k b n}\left(P_{1, n} f-P_{2, n} f\right)|\leq| f-P_{2, n} f \mid\right. \\
& +R_{k b n}\left|P_{1, n} f-P_{2, n} f\right| \leq C \omega(1 / n)+2^{-b n} 2 C(4 / \delta)^{l_{0} n} \omega(1 / n) \\
& \leq 3 C \omega(1 / n) .
\end{aligned}
$$

The inequalities (3.8)-(3.10) verify the lemma.

As usual, we identify a point $Q \in \mathbf{R}^{d}$ with its position vector $\overrightarrow{O Q}$, where $O$ is the origin.

Let $\Phi_{Q, \eta}$ be the homothetic transformation of $\mathbf{R}^{d}$ with center at $Q$ and with dilation factor $\eta$, i.e. $\Phi_{Q, \eta}$ is the linear transformation of $\mathbf{R}^{d}$ for which $\Phi_{Q, \eta}(P)=Q+\eta(P-Q)$ for any $P \in \mathbf{R}^{d}$.

Let $K$ be a convex polytope in $\mathbf{R}^{d}$ with vertices $Q_{1}, \ldots, Q_{m}$.

Lemma 3.6 $K$ is covered by the sets $\Phi_{Q_{j}, d /(d+1)} K, j=1, \ldots, m$.

Proof. By taking a simplicial decomposition ${ }^{2}$ of $K$ into $d$-dimensional simplices with vertices from the set $\left\{Q_{1}, \ldots, Q_{m}\right\}$, we may assume that $K$ is a simplex (so $m=d+1$ ). Then any point $X$ in $K$ is of the form $X=$ $\alpha_{1} Q_{1}+\cdots \alpha_{d+1} Q_{d+1}$ with $\alpha_{j} \geq 0, \sum \alpha_{j}=1$. Here one of the $\alpha_{j}$ 's, say $\alpha_{1}$, is at least $1 /(d+1)$, hence from the representation

$$
X=\left(1-\alpha_{1}\right)\left(\frac{\alpha_{2}}{1-\alpha_{1}} Q_{2}+\cdots+\frac{\lambda_{d+1}}{1-\alpha_{1}} Q_{d+1}\right)+\alpha_{1} Q_{1}=:\left(1-\alpha_{1}\right) Y+\alpha_{1} Q_{1}
$$

we can see that $X$ divides the segment $\overline{Y Q_{1}}$ into segments with length-ratio $\alpha_{1} /\left(1-\alpha_{1}\right) \geq 1 / d$, which implies that $X$ belongs to $\Phi_{Q_{1}, d /(d+1)} K$ because $Y \in K$.

\footnotetext{
${ }^{2}$ Actually, all we need is Caratheodory's theorem that any point of the convex hull of a set $H$ in $\mathbf{R}^{d}$ lies in the convex hull of some of its $(d+1)$ points, so if $K$ is a polytope in $\mathbf{R}^{d}$, then it is the union of the simplices spanned by $(d+1)$ vertices of $K$.
} 
Lemma 3.7 Let $K$ be as in the preceding lemma and let $0<\theta<1 /(d+1)$ be fixed. If $P$ is any point of $\Phi_{Q_{j}, 1-\theta} K$ and we translate $\Phi_{Q_{j}, \theta} K$ by the vector $\overrightarrow{Q_{j} P}$, then the translated polytope is part of $K$.

Proof. Indeed, if $R$ is any point of $\Phi_{Q_{j}, \theta} K$ and we denote the vectors $\overrightarrow{Q_{j} P}$ and $\overrightarrow{Q_{j} R}$ by $\underline{v}$ and $\underline{u}$, respectively, then $Q_{j}+(1-\theta)^{-1} \underline{v}, Q_{j}+\theta^{-1} \underline{u}$ belong to $K$, hence so does the point $(1-\theta)\left(Q_{j}+(1-\theta)^{-1} \underline{v}\right)+\theta\left(Q_{j}+\theta^{-1} \underline{u}\right)$ on their connecting segment. But this point is $Q_{j}+\underline{u}+\underline{v}=R+\underline{u}=R+\overrightarrow{Q_{j} P}$, which means that $\Phi_{Q_{j}, \theta} K+\overrightarrow{Q_{j} P} \subset K$. This proof also gives that if $P$ lies in the $d$ dimensional interior of $\Phi_{Q_{j}, 1-\theta} K$, then the translated polytope $\Phi_{Q_{j}, \theta} K+\overrightarrow{Q_{j} P}$ also lies in the interior of $K$.

What we have just proven can be applied on every lower dimensional face of $K$. Indeed, let $F_{k}$ be a $k$-dimensional face of $K$ that contains $Q_{j}$. Since $F_{k} \cap \Phi_{Q_{j}, \theta} K=\Phi_{Q_{j}, \theta} F_{k}$, what we have just established can be applied to the face $F_{k}$ and to $\Phi_{Q_{j}, \theta} F_{k}$. Thus, if $P \in \Phi_{Q_{j}, 1-\theta} K$ is on the face $F_{k}$, then the face $\Phi_{Q_{j}, \theta} F_{k}+\overrightarrow{Q_{j} P}$ of the translated polytope $\Phi_{Q_{j}, \theta} K+\overrightarrow{Q_{j} P}$ lies in $F_{k}$, and if $P$ lies in the $k$-dimensional interior of $\Phi_{Q_{j}, 1-\theta} F_{k}$, then $F_{k} \cap\left(\Phi_{Q_{j}, \theta} K+\overrightarrow{Q_{j} P}\right)$ lies in the $k$-dimensional interior of $F_{k}$. In the latter case if $P^{\prime}$ lies in the $k$ dimensional interior of $F_{k} \cap\left(\Phi_{Q_{j}, \theta} K+\overrightarrow{Q_{j} P}\right)$, then we can enlarge $\Phi_{Q_{j}, \theta} K+\overrightarrow{Q_{j} P}$ from $P^{\prime}$ by a factor $1+\tau>1$ with some small $\tau>0$ so that the enlarged polytope is still part of $K$. Furthermore, this enlarged polytope then contains the intersection of a neighborhood of $\Phi_{Q_{j}, \theta} K+\overrightarrow{Q_{j} P}$ with $F_{k}$ (note however, that it does not contain a $d$-dimensional neighborhood of $\Phi_{Q_{j}, \theta} K+\overrightarrow{Q_{j} P}$ since all points of $F_{k} \cap\left(\Phi_{Q_{j}, \theta} K+\overrightarrow{Q_{j} P}\right)$ lie on the boundary - actually on the corresponding $k$-dimensional face - of the enlarged polytope).

\subsection{Pyramidal coverings}

In this section we describe a covering of a polytope $K$ by pyramids $K_{s}$ with the property that there is a listing of the elements $K_{s}$ in the covering in which consecutive elements have relatively large intersections (the intersection of their interiors is non-empty). This covering will then allow us in the next subsection, by repeatedly applying the results from the preceding subsection, to obtain a global polynomial approximant on $K$ (to a given function $f$ ) from approximants on the $K_{s}$. In this way we reduce the problem of polynomial approximation on $K$ to approximation on (the) pyramids $\left(K_{s}\right)$.

Since the construction is rather involved with many notations, for the sake of the reader we shall list the notations in a separate subsection at the end of this section.

Let $K$ and $Q_{j}$ as before, and fix $0<\theta<1 /(d+1)$. Around each $Q_{j}$ we cut off from $K$ a small pyramid $S_{j}$ with vertex at $Q_{j}$ by a $(d-1)$-dimensional 


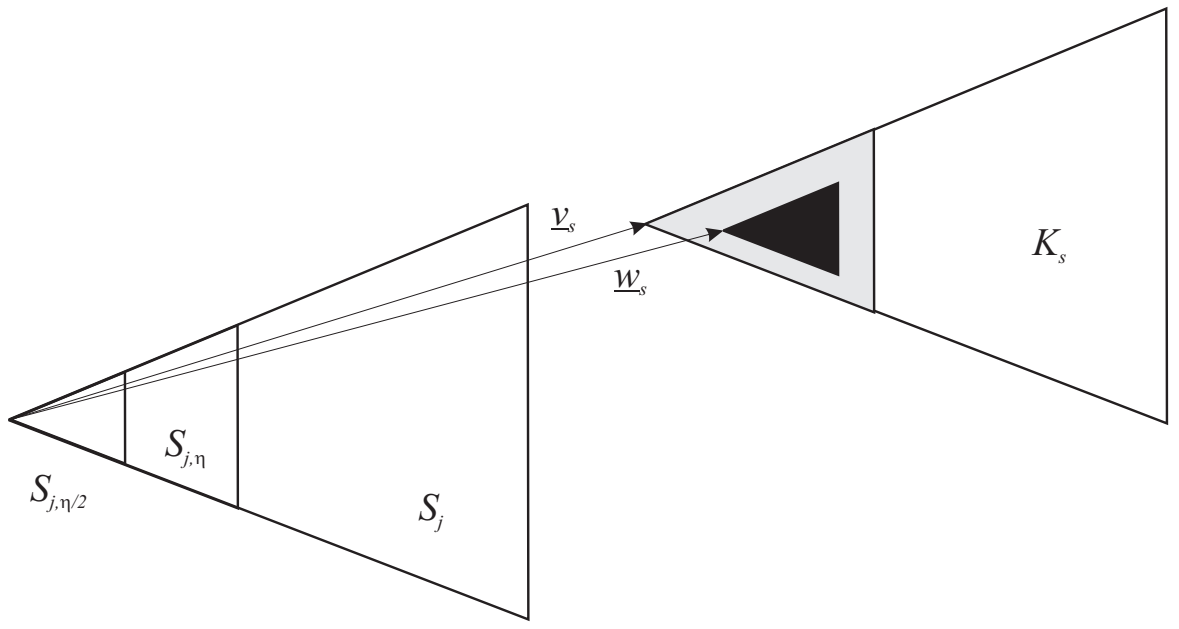

Figure 2: A schematic figure of the sets $S_{j}, S_{j, \eta}, S_{j, \eta / 2}, K_{s}, K_{\eta, s}$ (lightly shaded figure) and $\mathcal{K}_{\eta / 2, s}$ (dark shaded figure), as well as the vectors $\underline{v}_{s}, \underline{w}_{s}$.

hyperplane. We assume that for each $j$ we have $S_{j} \subset \Phi_{Q_{j}, \theta} K$, and we fix these $S_{j}$ in the following discussion.

For simplicity let us also write $S_{j, \eta}$ for the homothetic copy $\Phi_{Q_{j}, \eta} S_{j}$.

Let $\eta<1 / 2$ be given. We shall deal with the following type of sets $K_{s}, K_{\eta, s}$ and $\mathcal{K}_{\eta / 2, s}, 1 \leq s \leq T$, where $T$ will be given during the construction below.

- Each $K_{s}=S_{j}+\underline{v}_{s}$ is a translated copy of some $S_{j}$ by a translation vector $\underline{v}_{s}$,

- $K_{\eta, s} \subset S_{j, 1 / 2}+\underline{v}_{s}$ is the translation of the $\eta$-size copy $S_{j, \eta}$ of $S_{j}$ by the same vector $\underline{v}_{s}$, and

- $\mathcal{K}_{\eta / 2, s} \subseteq K_{\eta, s}$ is a translation of the smaller $S_{j, \eta / 2}$ by a possibly different vector $\underline{w}_{s}$.

See Figure 2. For emphasis we use calligraphic letter in $\mathcal{K}_{\eta / 2, s}$ to indicate that the vertex of $\mathcal{K}_{\eta / 2, s}$ may be at a different point than the common vertices of $K_{s}$ and $K_{\eta, s}$.

By an $\eta$-pyramid covering of $K$ we mean a covering $K=\cup_{s=1}^{T} K_{s}$, where each $K_{s}$ is a translated copy of one of the $S_{j}$ 's, and which has the following properties: there are an $\varepsilon>0$ and related subpyramids $K_{\eta, s}, \mathcal{K}_{\eta / 2, s}$ of $K_{s}$ as just described such that

(i) each $K_{s}$ is contained in $K$ and each $\mathcal{K}_{\eta / 2, s}$ is contained in $K_{\eta, s}$,

(ii) $K=\cup_{s=1}^{T} \mathcal{K}_{\eta / 2, s}$

(iii) the intersection of the interiors of $\mathcal{K}_{\eta / 2, s}$ and $\mathcal{K}_{\eta / 2, s+1}$ is non-empty for all $s=1, \ldots, T-1$, 
(iv) $\mathcal{K}_{\eta / 2, s}^{\varepsilon} \cap K \subset K_{\eta, s}$, where $\mathcal{K}_{\eta / 2, s}^{\varepsilon}$ is the closed $\varepsilon$-neighborhood of $\mathcal{K}_{\eta / 2, s}$.

Proposition 3.8 For every $\eta<1 / 2$ there is an $\eta$-pyramid covering of $K$.

Proof. We say that a finite set $X \subset K$ is $\tau$-dense in $K$ if every point of $K$ lies closer to some point of $X$ than $\tau$.

We define for some small $0<\tau$ a $\tau$-dense set $X$ on $K$ as follows. First of all, let $X_{1}$ be the set of all vertices $Q_{j}$ of $K$. Then for every edge $F_{1}$ of $K$ extend $F_{1} \cap X_{1}$ to a $\tau$-dense set of $F_{1}$. $X_{1}$ and the added points for all edges form $X_{2}$. Then for every 2 dimensional face $F_{2}$ of $K$ extend $F_{2} \cap X_{2}$ to a $\tau$-dense set of $F_{2}$, etc. keep extending the point sets $X_{k}$ using higher and higher dimensional faces. This way we get a $\tau$-dense set $X_{d-1}$ on the boundary of $K$, and finally extend that to a $\tau$-dense set on $K$.

First we define the sets $\mathcal{K}_{\eta / 2, s}$. If $P \in X$ and $P$ belongs to $\Phi_{Q_{j}, 1-\theta} K$ (there must be such a $j$ in view of Lemma 3.6) then let $K_{P}$ be the translation of $S_{j, \eta / 2}=\Phi_{Q_{j}, \eta / 2} S_{j}$ by the vector $\overrightarrow{Q_{j} P}$. If for a $P$ there are more than one $j$ with $P \in \Phi_{Q_{j}, 1-\theta} K$, then get a $K_{P}$ for all such $j$. We show that the collection of all these $K_{P}$ generate a suitable $\eta$-pyramid covering by repeating each $K_{P}$ a few times (to meet the requirement in (iii)). According to Lemma 3.7 we have $S_{j}+\overrightarrow{Q_{j} P} \subset K$ (recall that $\left.S_{j} \subset \Phi_{Q_{j}, \theta} K\right)$, and since $\eta<1 / 2$ we also have

$$
K_{P} \subset S_{j, 1 / 2}+\overrightarrow{Q_{j} P} \subset S_{j}+\overrightarrow{Q_{j} P} \subset K .
$$

If $\tau$ is sufficiently small, then these $K_{P}$ cover $K$. Indeed, from how $X$ was constructed it is clear that the vertices, and hence small neighborhoods (relative to $K$ ) of them are covered (note that each $Q_{j}$ belongs to $X$, so $S_{j, \eta / 2}$ is one of the $K_{P}$ 's). Then each edge $F_{1}$, and hence a small neighborhood (relative to $K$ ) of $F_{1}$ is covered (this neighborhood depends only on $\eta$ and not on $\tau$ ), and so on, we continue with higher and higher dimensional faces and their small neighborhoods, and finally with all of $K$. This proves (ii).

We also get that for small $\tau>0$ the interiors $\operatorname{Int}\left(K_{P}\right)$ of the pyramids $K_{P}$ cover the interior $\operatorname{Int}(K)$ of $K$. At this point we need the following simple lemma.

Lemma 3.9 Let $\mathcal{G}$ be a connected set and suppose that $\mathcal{G}=\cup_{i} \mathcal{G}_{i}$, where $\mathcal{G}_{i}$ are finitely many open sets. Let $\left\{\mathcal{G}_{i}\right\}_{i}$ be the vertices of a graph, and connect in that graph the vertices $\mathcal{G}_{i}$ and $\mathcal{G}_{j}$ if for the sets $\mathcal{G}_{i}, \mathcal{G}_{j}$ we have $\mathcal{G}_{i} \cap \mathcal{G}_{j} \neq \emptyset$. Then this graph is connected.

This lemma says that in any covering $\mathcal{G}=\cup_{i} \mathcal{G}_{i}$ of a connected open set $\mathcal{G}$ by open sets $\mathcal{G}_{i}$, for any index pairs $k \neq l$ there are indices $i_{0}, i_{j}, \ldots, i_{m}$ with some $m$ such that $i_{0}=k, i_{m}=l$, and for any $0 \leq j<m$ we have $\mathcal{G}_{i_{j}} \cap \mathcal{G}_{i_{j+1}} \neq \emptyset$.

Proof. For clearer notation let us write $\mathcal{G}_{j}^{V}$ if we talk about $\mathcal{G}_{j}$ as a vertex of the graph. Let $V_{1}, \ldots, V_{r}$ be the vertex sets of the connected components of the graph (i.e. each $V_{s}$ is a non-empty set of certain $\mathcal{G}_{j}^{V}, V_{s_{1}} \cap V_{s_{2}}=\emptyset$ for $s_{1} \neq s_{2}$, 
and each $\mathcal{G}_{j}^{V}$ belongs to one of the $V_{s}$ ). Each $\cup_{\mathcal{G}_{j}^{V} \in V_{i}} \mathcal{G}_{j}$ is open, and for different components $V_{i}$ these sets are disjoint. Now the conclusion follows from the fact that a connected set cannot be the disjoint union of non-empty open sets.

Since the just constructed graph for $\operatorname{Int}(K)$ and $\left\{\operatorname{Int}\left(K_{P}\right)\right\}_{P \in X}$ is connected, there is a path in it which goes through every vertex. In other words, there is a listing $\mathcal{K}_{\eta / 2, s}, s=1, \ldots, T$, of all the $K_{P}$ 's (maybe with repetition) in which $\mathcal{K}_{\eta / 2, s} \cap \mathcal{K}_{\eta / 2, s+1}$ contains a ball for all $s<T$. This proves (iii).

We still need to define the sets $K_{\eta, s}$ and $K_{s}$, see Figure 2. Consider a $\mathcal{K}_{\eta / 2, s}=K_{P}$, say it is $S_{j, \eta / 2}+\overrightarrow{Q_{j} P}$ (i.e. the vector $\underline{w}_{s}$ in the definition of pyramidal covering is $\overrightarrow{Q_{j} P}$ in this case). If $P$ is the vertex $Q_{j}$, then enlarge $K_{P}$ from $P$ by a factor 2 to get $K_{\eta, s}$. However, if $P$ is not a vertex of $K$, then there is a smallest dimensional face $F_{k}$ of $K$ such that $P$ lies in the interior $\operatorname{Int}_{k}\left(F_{k}\right)$ of $F_{k}$ (if $F_{k}$ is $k$-dimensional, then $\operatorname{Int}_{k}\left(F_{k}\right)$ denotes its $k$-dimensional interior), where we consider $K$ itself as the $d$-dimensional face of $K$. Hence, according to what was said after Lemma $3.7, F_{k} \cap\left(S_{j, \eta}+\overrightarrow{Q_{j} P}\right)$ also lies in $\operatorname{Int}_{k}\left(F_{k}\right)$. Let $Q \in \operatorname{Int}_{k}\left(F_{k} \cap S_{j, \eta}\right)$ be a point lying in the $k$-dimensional interior of $F_{k} \cap S_{j, \eta}$. Then $Q+\overrightarrow{Q_{j} P}$ lies in the $k$-dimensional interior $\operatorname{Int}_{k}\left(F_{k} \cap\left(S_{j, \eta}+\overrightarrow{Q_{j} P}\right)\right)$ of the face $F_{k} \cap\left(S_{j, \eta}+\overrightarrow{Q_{j} P}\right)$ of $S_{j, \eta}+\overrightarrow{Q_{j} P}$, and the translation of $\operatorname{Int}_{k}\left(F_{k} \cap\left(S_{j, \eta}+\overrightarrow{Q_{j} P}\right)\right)$ by the vector $\overrightarrow{Q Q_{j}}$ will contain $F_{k} \cap K_{P}$ if $Q$ lies sufficiently close to $Q_{j}$ (and at the same time this translation is contained in $F_{k}$ ). Thus, if we set

$$
K_{\eta, s}=S_{j, \eta}+\overrightarrow{Q P}
$$

(i.e. the vector $\underline{v}_{s}$ in the pyramidal covering is $\overrightarrow{Q P}$ ), then this $K_{\eta, s}$ is such that it contains the intersection of a neighborhood of $\mathcal{K}_{\eta / 2, s}=K_{P}$ with $K$, proving (iv).

Finally, with the previous notations we set $K_{s}=S_{j}+\overrightarrow{Q P}$, which is part of $K$ (see Lemma 3.7 ), so (i) holds.

\section{List of notations used in the pyramidal covering construction}

For the sake of the reader we repeat here in a concised form the notations used in the pyramidal covering.

$\Phi_{Q, \eta}$ - homothetic transformation with center at $Q$ and dilation factor $\eta$

$Q_{j}$ - vertices of $K$

$S_{j}(\subset K)$ - small pyramids cut off from $K$ with vertex at $S_{j}$

$S_{j, \eta}$ - the homothetic copy $\Phi_{Q_{j}, \eta} S_{j}$ of $S_{j}$

$K_{s}-$ an $S_{j}+\underline{v}_{s}$ with a translation vector $\underline{v}_{s}$

$K_{\eta, s}-S_{j, \eta}+\underline{v}_{s}$ with the same translation vector $\underline{v}_{s}$

$\mathcal{K}_{\eta / 2, s}\left(\subset K_{\eta, s}\right)-S_{j, \eta / 2}+\underline{w}_{s}$ with another translation vector $\underline{w}_{s}$ 


\subsection{Patching approximants on a pyramidal covering to- gether to a global approximant}

Let $\tilde{\omega}_{S_{j}}^{r}(f, 1 / n)$ be the modulus of smoothness on $S_{j}$ when only the directions in the apex edges of $S_{j}$ are used. We shall use again the notation $S_{j, \eta}$ for $\Phi_{Q_{j}, \eta} S_{j}$.

Proposition 3.10 If for each $j=1, \ldots, m$ we have

$$
E_{n}^{L}(f)_{S_{j, 2 \eta}} \prec \tilde{\omega}_{S_{j}}^{r}(f, 1 / n), \quad f \in C\left(S_{j}\right),
$$

then there is an l such that

$$
E_{l n}^{L}(f)_{K} \prec \omega_{K}^{r}(f, 1 / n), \quad f \in C(K) .
$$

Recall that here, on the right hand side, the modulus of smoothness uses the edge directions of $K$.

Proof. Let $K=\cup_{s=1}^{T} K_{s}$ be an $\eta$-pyramid covering of $K$ as in Proposition 3.8, and set $H_{t}=\cup_{s=1}^{t} \mathcal{K}_{\eta / 2, s}$. With induction we are going to prove that for every $1 \leq t \leq T$ there is an $l$ for which

$$
E_{l n}^{L}(f)_{H_{t}} \prec \omega_{K}^{r}(f, 1 / n) .
$$

The claim then follows by setting $t=T$.

For $t=1$ the set $H_{1}=\mathcal{K}_{\eta / 2,1}$ is an $S_{j, \eta / 2}+\underline{w}_{1}$ for some $j$ and some vector $\underline{w}_{1}, K_{1}=S_{j}+\underline{v}_{1}$ with the same $j$ and with another vector $\underline{v}_{1}$, and the corresponding $K_{\eta, 1}$ is $S_{j, \eta}+\underline{v}_{1}$. Note that $K_{\eta, 1}$ is contained in $S_{j, 1 / 2}+\underline{v}_{1}$. By the assumption (make a homothecy with factor 2 and a translation by $\underline{v}_{1}$ ) there are linear operators $L_{n}: C\left(S_{j, 1 / 2}+\underline{v}_{1}\right) \rightarrow \Pi_{n}^{d}$ such that

$$
\left\|f-L_{n} f\right\|_{S_{j, \eta}+\underline{v}_{1}} \prec \tilde{\omega}_{S_{j, 1 / 2}+\underline{v}_{1}}^{r}(f, 1 / n) .
$$

Since $\mathcal{K}_{\eta / 2,1}=S_{j, \eta / 2}+\underline{w}_{1}$ is part of $S_{j, \eta}+\underline{v}_{1}$ (see property (i) of the $\eta$-pyramid covering), and the right-hand side in (3.14) is at most $\omega_{K}^{r}(f, 1 / n)$ (note that all apex-edge directions of $S_{j}+\underline{v}_{1}$ are also edge directions of $\left.K\right)$, we obtain

$$
\left\|f-L_{n} f\right\|_{\mathcal{K}_{\eta / 2,1}}=\left\|f-L_{n} f\right\|_{S_{j, \eta / 2}+\underline{w}_{1}} \prec \omega_{K}^{r}(f, 1 / n),
$$

and hence the claim follows in this case.

The induction step is a consequence of Lemma 3.4. Indeed, if (3.13) is known for $t-1(t>1)$, say

$$
E_{l_{0} n}^{L}(f)_{H_{t-1}} \prec \omega_{K}^{r}(f, 1 / n),
$$

then let $H=H_{t-1}, S=\mathcal{K}_{\eta / 2, t}$ in Lemma 3.4 , and set $\omega(1 / n)=\omega_{K}^{r}(f, 1 / n)$ there (note that by property (iii) of the $\eta$-pyramidal covering $H \cap S=H_{t-1} \cap \mathcal{K}_{\eta / 2, t} \supseteq$ $\mathcal{K}_{\eta / 2, t-1} \cap \mathcal{K}_{\eta / 2, t}$ contains a ball). As we have just seen (see the argument leading to $(3.14)-(3.15))$, (3.12) implies

$$
E_{n}^{L}(f)_{K_{\eta, t}} \prec \omega_{K}^{r}(f, 1 / n) .
$$


Furthermore, by property (iv) of an $\eta$-pyramid covering, for some $\varepsilon>0$ the relative (with respect to $K$ ) neighborhood $\mathcal{K}_{\eta / 2, t}^{\varepsilon} \cap K$ of $\mathcal{K}_{\eta / 2, t}$ is part of $K_{\eta, t}$, hence we obtain

$$
E_{n}^{L}(f)_{\mathcal{K}_{\eta / 2, t}^{\varepsilon} \cap K} \prec \omega_{K}^{r}(f, 1 / n),
$$

and together with this also

$$
E_{l_{0} n}^{L}(f)_{\mathcal{K}_{\eta / 2, t}^{\varepsilon} \cap K} \prec \omega_{K}^{r}(f, 1 / n) .
$$

Now the claim follows for $t$ (i.e. for $H_{t}=H_{t-1} \cup \mathcal{K}_{\eta / 2, t}$ ) in view of this estimate, (3.16) and Lemma 3.4.

\section{Approximation on pyramids and the proof of Theorem 1.1 for large degrees}

Let $S_{0}$ be a $(d-1)$-dimensional convex polytope lying in the hyperplane $H$ of $\mathbf{R}^{d}$ and $S$ the convex hull of $S_{0} \cup\{P\}$, where $P$ is a point outside $H$ (so $S$ is a $d$ dimensional pyramid with apex at $P$ ). Recall that the edges of $S$ emanating from $P$ are called apex edges.

Assume that $P=0$. Let $\lambda H$ be the hyperplane that we obtain from $H$ by a dilation with factor $0<\lambda<1$ and center $P=0$. Then $\lambda H$ cuts $S$ into two parts, the pyramid $\lambda S$ and the polytope $U_{\lambda}:=S \backslash \lambda S$ such that $S_{0}$ and $\lambda S_{0}$ are $(d-1)$-dimensional similar faces of $U_{\lambda}$. Let $e_{1}, \ldots, e_{m}$ be the segments connecting the corresponding points of $S_{0}$ and $\lambda S_{0}$. These segments lie on the apex edges of $S$, on each apex edge lying one of them. If $E$ is the endpoint of $e_{j}$ lying in $\lambda S_{0}$, then the product set $\left(\lambda S_{0}\right) \times e_{j}$ is defined as the union of all the translations $e_{j}+\overrightarrow{E M}$, where $M$ runs through the points of $\lambda S_{0}$ (see Figure 3).

Proposition 4.1 If $\lambda>(d-1) / d$, then the product sets $\left(\lambda S_{0}\right) \times e_{j}, 1 \leq j \leq m$ have a common interior and their union is $U_{\lambda}$.

Proof. Let the vertices of $S_{0}$ be $Q_{1}, \ldots, Q_{m}$. Then the vertices of $\lambda S$ are $\lambda Q_{1}, \ldots, \lambda Q_{m}$, and we may assume that $e_{j}=\overline{Q_{j}\left(\lambda Q_{j}\right)}$. As before, let $\Phi_{Q_{j}, \mu} S_{0}$ be the homothetic image of $S_{0}$ with factor $\mu$ and dilation center at $Q_{j}$.

Let $Z$ be any point in $U_{\lambda}$, and $\ell_{Z}$ be the half-line from the origin through $Z$. This $\ell_{Z}$ intersects $S_{0}$ in a point $X$, and by Lemma $3.6 X \in \Phi_{Q_{j},(d-1) / d} S_{0}$ for some $1 \leq j \leq m$. If $Z=\sigma X$, then $\lambda \leq \sigma \leq 1$, and $Z \in \Phi_{\sigma Q_{j},(d-1) / d}\left(\sigma S_{0}\right)$. However, this latter set is a $\sigma(d-1) / d$ size copy $\left(\Phi_{Q_{j}, \sigma(d-1) / d}\left(S_{j}\right)\right)$ of $S_{0}$ translated by the vector $\overrightarrow{Q_{j}\left(\sigma Q_{j}\right)}$, so it is contained in the $\lambda>\sigma(d-1) / d$ size copy $\Phi_{Q_{j}, \lambda} S_{0}$ of $S_{0}$ translated by the same vector $\overrightarrow{Q_{j}\left(\sigma Q_{j}\right)}$.

The translation of $\lambda S_{0}$ by the vector $\overrightarrow{\left(\lambda Q_{j}\right) Q_{j}}$ is $\Phi_{Q_{j}, \lambda} S_{0}$, and the product set $\left(\lambda S_{0}\right) \times e_{j}$ is precisely the set of points that lie in one of the translations of $\lambda S_{0}$ by a vector $\overrightarrow{\left(\lambda Q_{j}\right)\left(\tau Q_{j}\right)}, \lambda \leq \tau \leq 1$. But then, in view of $\overrightarrow{Q_{j}\left(\sigma Q_{j}\right)}=$ 


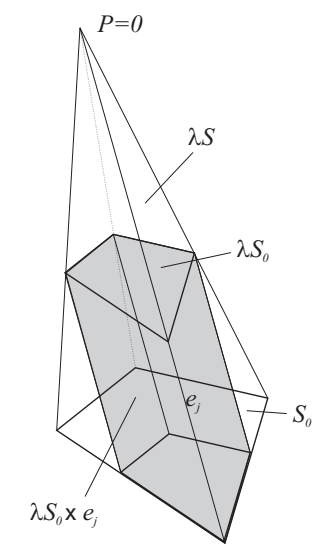

Figure 3: The pyramid $S$, its base $S_{0}$ and their $\lambda$-dilations from the apex $P=0$. The shaded slab is the product set $\lambda S_{0} \times e_{j}$.

$\overrightarrow{Q_{j}\left(\lambda Q_{j}\right)}+\overrightarrow{\left(\lambda Q_{j}\right)\left(\sigma Q_{j}\right)}$, what we have just proven means that $Z$ is in this product set $\left(\lambda S_{0}\right) \times e_{j}$.

The claim that the product sets $\left(\lambda S_{0}\right) \times e_{j}$ have a common interior point is trivial, since if $Y$ lies in the $(d-1)$-dimensional interior of $\lambda S_{0}$, then all points of $U_{\lambda}=S \backslash \lambda S$ that lie sufficiently close to $Y$ lie in all the product sets $\left(\lambda S_{0}\right) \times e_{j}$.

Remark 4.2 Easy modification of the proof gives that if $1>\lambda>(d-1) / d$ and $\mu\langle 1, \mu \lambda\rangle(d-1) / d$, then for $\mu$ sufficiently close to 1 even the product sets $\Phi_{\lambda Q_{j}, \mu}\left(\lambda S_{0}\right) \times e_{j}, 1 \leq j \leq m$ have a common interior and their union is $U_{\lambda} \cdot{ }^{3}$ For these sets it is clearly true that for small $\varepsilon>0$

$$
\left(\Phi_{\lambda Q_{j}, \mu}\left(\lambda S_{0}\right) \times e_{j}\right)^{\varepsilon} \cap U_{\lambda} \subset\left(\lambda S_{0}\right) \times e_{j},
$$

where ${ }^{\varepsilon}$ denotes $\varepsilon$-neighborhood.

Proposition 4.3 Suppose that $S_{0}$ is a polytope in $\mathbf{R}^{d-1}$ for which there are bounded linear operators $V_{n}: C\left(S_{0}\right) \rightarrow \Pi_{n}^{d-1}$ such that

$$
\left\|f-V_{n} f\right\|_{S_{0}} \prec \omega_{S_{0}}^{r}(f, 1 / n)_{\mathcal{E}}
$$

\footnotetext{
${ }^{3}$ Actually, the claim is true for all $\mu<1$ with $\mu \lambda>(d-1) / d$. Indeed, in view of Proposition 4.1 we need to prove only that the convex sets $\Phi_{\lambda Q_{j},(d-1) / d} \lambda S_{0}$ have non-empty intersection. For this the following simple argument was communicated to the author by János Kincses. In view of Helly's theorem all these sets have non-empty intersection if any $d$ of them have nonempty intersection (note that we are working now in a $d-1$ dimensional hyperplane of $\mathbf{R}^{d}$ ). However, if $j_{1}, \ldots, j_{d}$ are different indices, then the center of mass of the $((d-1)$-dimensional $)$ simplex $\Delta$ determined by $\lambda Q_{j_{1}}, \ldots, \lambda Q_{j_{d}}$ lies in all $\Phi_{\lambda Q_{j_{s}},(d-1) / d} \lambda S_{0}, s=1, \ldots, d$, because the center of mass divides the segment from any vertex $Q_{j_{s}}$ through the center of mass to the opposite face of $\Delta$ in the ration $1:(d-1)$.
} 
where $\omega_{S_{0}}^{r}(f, \delta)_{\mathcal{E}}$ is a modulus of smoothness on $S_{0}$ using some directions $\mathcal{E}$. Then there is a sequence of bounded linear operators $L_{n}: C\left(S_{0} \times[0,1]\right) \rightarrow \Pi_{n}^{d}$ such that

$$
\left\|f-L_{n} f\right\|_{S_{0} \times[0,1]} \prec \omega_{S_{0} \times[0,1]}^{r}(f, 1 / n)_{\mathcal{E} \cup\left\{e_{d}\right\}},
$$

where $e_{d}$ is the d-th unit vector in $\mathbf{R}^{d}$ (perpendicular to $\mathbf{R}^{d-1}$ ).

Proof. In view of Remark 1.2 it is sufficient to construct the linear operators $L_{n}$ with the required properties except that $L_{n} f$ can be a polynomial of degree at most $n$ in each variable (as opposed to the claim where $L_{n} f$ has total degree at $\operatorname{most} n$ ).

This is basically [6, Section 12.1]. Indeed, it was proven there that for every $r$ there are $U_{n}: C[0,1] \rightarrow \Pi_{n}^{1}$ such that $U_{n}$ are uniformly bounded linear operators from $C[0,1]$ to $C[0,1]$ with the property that

$$
\left\|f-U_{n} f\right\|_{[0,1]} \prec \omega_{[0,1]}^{r}(f, 1 / n)
$$

(recall also (1.7)).

Let $y=\left(y_{1}, \ldots, y_{d-1}\right)$. For a function $f(y, x)$ define $f_{x}(y)=f(y, x)$ and $f^{\underline{y}}(x)=f(\underline{y}, x)$. Then

$$
\omega_{S_{0} \times[0,1]}(f, \delta)_{\mathcal{E} \cup\left\{e_{d}\right\}}=\max \left(\sup _{x \in[0,1]} \omega_{S_{0}}\left(f_{x}, \delta\right)_{\mathcal{E}}, \sup _{\underline{y} \in S_{0}} \omega_{[0,1]}(f \underline{y}, \delta)\right) .
$$

When we apply $V_{n}$ in the variable $y \in S_{0}$, then we denote it by $V_{n}^{\underline{y}}$. Let now $L_{n} f=U_{n}^{x} \circ V_{n}^{\underline{y}} f_{x}$. The function $V_{n}^{\underline{y}} f_{x}$ is of the form $\sum_{k_{i} \leq n} a_{k, n}(x) y_{1}^{k_{1}} \cdots y_{d-1}^{k_{d-1}}$ where $\underline{k}=\left(k_{1}, \ldots, k_{d-1}\right)$, and here each $a_{\underline{k}, n}(x)$ is continuous because $V_{n}$ are bounded linear operators. Hence, the linearity of $U_{n}$ yields that $L_{n} f$ is of the form $\sum_{k_{i} \leq n} U_{n}^{x}\left(a_{\underline{k}, n}(x)\right) y_{1}^{k_{1}} \cdots y_{d-1}^{d_{d-1}}$, which is a polynomial of degree at most $n$ in each of the variables $y_{1}, \ldots, y_{d-1}, x$. Now

$$
\left|L_{n} f(\underline{y}, x)-f(\underline{y}, x)\right| \leq\left|U_{n}^{x} \circ V_{n}^{y} f_{x}(\underline{y})-U_{n}^{x} f_{x}(\underline{y})\right|+\left|U_{n}^{x} f_{x}(\underline{y})-f_{x}(\underline{y})\right| .
$$

With some fixed $C, C_{1}, C_{2}$ for all large $n$ the first term on the right is

$$
\begin{aligned}
\left|U_{n}^{x} \circ V_{n}^{\underline{y}} f_{x}(\underline{y})-U_{n}^{x} f_{x}(\underline{y})\right| & =\left|U_{n}^{x}\left(V_{n}^{\underline{y}} f_{x}(\underline{y})-f_{x}(\underline{y})\right)\right| \\
& \left.\leq C \sup _{x} \mid V_{n}^{\underline{y}} f_{x}(\underline{y})-f_{x}(\underline{y})\right) \mid \\
& \leq C_{1} \sup _{x} \omega_{S_{0}}\left(f_{x}, 1 / n\right)_{\mathcal{E}} \leq C_{1} \omega_{S_{0} \times[0,1]}(f, 1 / n)_{\mathcal{E} \cup\left\{e_{d}\right\}},
\end{aligned}
$$

while for each fixed $y \in S_{0}$ the second term is

$$
\left|U_{n}^{x} f \underline{y}(x)-f \underline{y}(x)\right| \leq C_{2} \omega_{[0,1]}\left(f^{\underline{y}}, 1 / n\right) \leq C \omega_{S_{0} \times[0,1]}(f, 1 / n)_{\mathcal{E} \cup\left\{e_{d}\right\}} .
$$


The preceding proposition says that if we can prove a Jackson-type inequality on a set $K_{0}$ via bounded linear operators, then a similar Jackson-type theorem holds on the product space $K_{0} \times[0,1]$. By applying an affine transformation we obtain that the same is true for product sets of the form $K_{0} \times e$, where $e$ is not necessarily perpendicular to the hyperspace of $K_{0}$ (but lies outside it). In particular, we can apply this to all the product sets $\left(\lambda S_{0}\right) \times e_{j}$ from Proposition 4.1 above, and we obtain that if

$$
E_{n}^{L}(f)_{S_{0}} \prec \omega_{S_{0}}^{r}(f, 1 / n),
$$

(which implies the same for the set $\lambda S_{0}$ replacing $S_{0}$ since $\lambda S_{0}$ is a homothetic image of $\left.S_{0}\right)$ where $\omega_{S_{0}}^{r}(f, \delta)$ is the modulus of smoothness on $S_{0}$ using the edge directions $S_{0}$, then for each $j=1, \ldots, m$ we have

$$
E_{n}^{L}(f)_{\left(\lambda S_{0}\right) \times e_{j}} \prec \omega_{\left(\lambda S_{0}\right) \times e_{j}}^{r}(f, 1 / n) .
$$

Let us return now to the pyramid $S$ and to its decomposition $S=\lambda S \cup U_{\lambda}$ from the beginning of this section. If we combine the just discussed relation with the fact (4.1) and apply Lemma 3.4 repeatedly with $\omega(1 / n)=\omega_{U_{\lambda}}^{r}(f, 1 / n)$, $H=\cup_{j=1}^{t-1} \Phi_{\lambda Q_{j}, \mu}\left(\lambda S_{0}\right) \times e_{j}, S=\Phi_{\lambda Q_{t}, \mu}\left(\lambda S_{0}\right) \times e_{t}, t=1, \ldots, m$, and $K=U_{\lambda}$, then we can conclude that (4.2) implies that there is an $l$ such that

$$
E_{l n}^{L}(f)_{U_{\lambda}} \prec \omega_{U_{\lambda}}^{r}(f, 1 / n) .
$$

If this is true for all $n$ with $l n \geq n_{r}$ (where $n_{r}$ is some number), then we have proved

Proposition 4.4 Under the assumption (4.2) there is an $n_{r}$ such that

$$
E_{n}^{L}(F)_{U_{\lambda}} \prec \omega_{U_{\lambda}}^{r}(F, 1 / n), \quad n \geq n_{r} .
$$

Recall our convention that this means that for all functions $F$ continuous on $U_{\lambda}$ we have

$$
E_{n}^{L}(F)_{U_{\lambda}} \leq C_{0} \omega_{U_{\lambda}}^{r}(F, 1 / n), \quad n \geq n_{r}
$$

with some constant $C_{0}$ independent of $F$.

Since both sides are homothetic invariant, we also have

$$
E_{b n}^{L}(F)_{q U_{\lambda}} \prec \omega_{q U_{\lambda}}^{r}(F, 1 / b n), \quad b n \geq n_{r} .
$$

for any $q>0$ and $b>0$.

In particular, if $\mathcal{E}$ is the set of the apex edge directions of $S$ and for some function $F$ and numbers $q, b>0$

$$
\omega_{q U_{\lambda}}^{r}(F, 1 / b n) \prec \omega_{S}^{r}(f, 1 / n)_{\mathcal{E}}
$$

also holds, then we can conclude

$$
E_{b n}^{L}(F)_{q U_{\lambda}} \prec \omega_{S}^{r}(f, 1 / n)_{\mathcal{E}}, \quad b n \geq n_{r} .
$$

Now we are ready to prove Theorem 1.1 for large degrees $n$.

We shall prove it in the form 
Theorem 4.5 If $K$ is a convex polytope in $\mathbf{R}^{d}$, then

$$
E_{n}^{L}(f)_{K} \prec \omega_{K}^{r}(f, 1 / n) .
$$

Recall our convention from Section 3 that $a_{n} \prec b_{n}$ means that there is a $C>0$ such that for sufficiently large $n$ we have $\left|a_{n}\right| \leq C b_{n}$.

Proof of Theorem 4.5. We use induction with respect to $d$, the $d=1$ case being covered by [6, Section 12.1]. Suppose the claim is true in $\mathbf{R}^{d-1}$, and let $K$ be a polytope in $\mathbf{R}^{d}$.

Let $Q$ be a vertex of $K$, and cut off a small pyramid $S$ from $K$ by a hyperplane going close to $Q$. We may assume that $S$ is as small as in the discussion after Lemma 3.6, i.e. $S \subset \Phi_{Q, \theta} K$ (where $\theta<1 /(d+1)$ is a fixed number). The apex of $S$ is at $Q$ and its base $S_{0}$ is in the hyperplane of the cut. It was proved in [14, Chapter 11] part A) that the cut can be made so that

a) no base edge $e$ of $S$ is parallel with any $(d-2)$-dimensional base face not containing $e$,

b) the height of $S$ (i.e. the perpendicular segment to $S_{0}$ from $Q$ ) lies in the interior of $S$ (except for its two endpoints).

We may assume that $Q=0$, and that $S_{0}$ lies in the plane $x_{1}=2$ (implying that the height is the segment $\left.0 \leq x_{1} \leq 2, x_{2}=\cdots x_{d}=0\right)$, and let $K_{a}=$ $a S \backslash(a / 4) S$. Properties a)-b) are enough to prove (see [14, Chapter 6]) that for $a \leq 1 / 4$ we have

$$
\omega_{K_{a}}^{r}(F, 1 / n \sqrt{a}) \prec \omega_{S}^{r}(f, 1 / n)_{\mathcal{E}}
$$

where $\mathcal{E}$ is the set of the apex edge directions of $S$ and where

$$
F\left(x_{1}, \ldots, x_{d}\right)=f\left(x_{1}+L / n^{2}, x_{2}, \ldots, x_{d}\right)
$$

with some large but fixed $L$ (in [14, (6.18)] this was stated with a different modulus of smoothness on the right which was needed there, but the proof also gives the inequality with $\left.\omega_{S}^{r}(f, \delta)_{\mathcal{E}}\right)$. As a consequence, we get the same conclusion if $K_{a}$ is replaced by $a S \backslash(\lambda a) S=a U_{\lambda}$ with any $1 / 4 \leq \lambda<1$, where $U_{\lambda}$ is the set from Proposition 4.4, i.e.

$$
\omega_{a U_{\lambda}}^{r}(F, 1 / n \sqrt{a}) \prec \omega_{S}^{r}(f, 1 / n)_{\mathcal{E}}
$$

also follows.

Now this is then a situation when (4.4) can be applied with $q=a, b=\sqrt{a}$ since (4.2) is guaranteed by our induction hypothesis and (4.7) is the same as (4.3), so we can conclude

$$
E_{n \sqrt{a}}^{L}(F)_{a U_{\lambda}} \prec \omega_{S}^{r}(f, 1 / n)_{\mathcal{E}} \quad n \sqrt{a} \geq n_{r} .
$$

Recall that here $F$ and $f$ are connected via (4.6). These local approximants can be patched together to a global approximant exactly as in [14, Chapter 8]. Namely, if

$$
S_{n}^{*}=S \cap\left\{M / n^{2} \leq x_{1} \leq 1 / 16\right\}
$$


with some appropriate large but fixed $M$, then (4.8) implies that for some $l$ we have

$$
E_{l n}^{L}(F)_{S_{n}^{*}} \prec \omega_{S}^{r}(f, 1 / n)_{\mathcal{E}} .
$$

The only difference here compared to $[14$, Chapter 8$]$ is that there $\lambda=1 / 4$ was used (in which case $a U_{\lambda}=K_{a}$ with the $K_{a}$ from (4.5)), but for any $\frac{1}{4} \leq \lambda<1$ the argument is the same.

Since $\omega_{S}^{r}(f, 1 / n)_{\mathcal{E}} \prec \omega_{S}^{r}(f, 1 / \ln )_{\mathcal{E}}$, it follows that

$$
E_{n}^{L}(f)_{(S / 64)+\left((M+L) / n^{2}\right) \underline{v}} \prec \omega_{S}^{r}(f, 1 / n)_{\mathcal{E}},
$$

where $\underline{v}=(1,0, \ldots, 0)$ is the unit vector pointing in the $x_{1}$-direction. This conclusion is immediate, since $E_{n}^{L}(F)_{S_{n}^{*}}=E_{n}^{L}(f)_{S_{n}^{*}+\left(L / n^{2}\right) v}$, and the set $S_{n}^{*}+$ $\left(L / n^{2}\right) \underline{v}$ contains $(S / 64)+\left((M+L) / n^{2}\right) \underline{v}$ for large $n$.

Now we invoke

Proposition 4.6 (4.9) implies

$$
E_{n}^{L}(f)_{S / 128} \prec \omega_{S}^{r}(f, 1 / n)_{\mathcal{E}}
$$

The proof of this will be given after we complete the proof of Theorem 4.5.

Thus, we can see that

$$
E_{n}^{L}(f)_{S_{2 \eta}} \prec \omega_{S}^{r}(f, 1 / n)_{\mathcal{E}}
$$

with any $2 \eta<1 / 128$ (recall that $S_{2 \eta}$ is the dilation of $S$ from its apex by the factor $2 \eta$ ), and this is precisely (3.12) for the vertex $Q$ and the pyramid $S$ attached to $Q$.

Since this can be done for all vertices $Q$ of $K$, the claim in the theorem follows from Proposition 3.10.

We still need to prove Proposition 4.6.

Proof. Recall that $\mathcal{E}$ is the direction of the apex edges of $S$ and $\underline{v}$ is the vector $(1,0, \ldots, 0)$. It is enough to show that if $p_{n}$ is any polynomial such that

$$
\left\|f-p_{n}\right\|_{(S / 64)+\left(M / n^{2}\right) \underline{v}} \leq C_{0} \omega_{S}^{r}(f, 1 / n)_{\mathcal{E}}
$$

with some fixed $M$, then automatically

$$
\left\|f-p_{n}\right\|_{S / 128} \leq C_{1} \omega_{S}^{r}(f, 1 / n)_{\mathcal{E}}
$$

with some $C_{1}$ that is independent of $n$ (in the Proposition apply this with $M+L$ instead of $M$ and with $p_{n}=L_{n} f$ ). As before, let $S_{0}$ be the base of $S, Q_{1}, \ldots, Q_{m}$ the vertices of $S_{0}$ and $\underline{e}_{j}=\overrightarrow{0 Q_{j}}$ the vectors from the apex 0 of $S$ (which is at the origin) to the base vertices. The vector pointing from the origin to the apex of $S / 64+\left(M / n^{2}\right) \underline{v}$ is $\left(M / n^{2}\right) \underline{v}=\left(M / n^{2}, 0, \ldots, 0\right)$, and first we express this in 


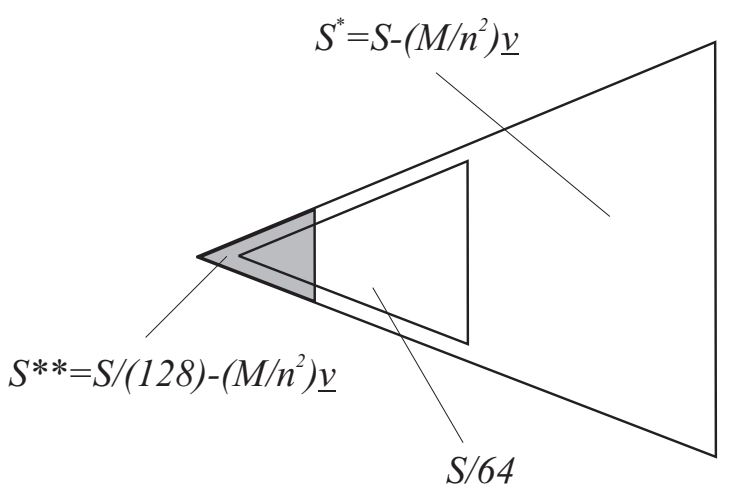

Figure 4: A schematic figure of the sets $S / 64, S^{*}:=S-\left(M / n^{2}\right) v$ and $S^{* *}:=$ $S / 128-\left(M / n^{2}\right) v$ (shaded area)

terms of the vectors $\underline{e}_{j}$. Recall that the base of $S$ is on the $x_{1}=2$ hyperplane, and the point $(2,0, \ldots, 0)$ is a point of the base (because the height of $S$ was lying inside $S$ ). Thus, with some numbers $\alpha_{j} \in[0,1]$ with total sum 1 we have

$$
\alpha_{1} \underline{e}_{1}+\cdots+\alpha_{m} \underline{e}_{m}=(2,0, \ldots, 0),
$$

hence

$$
\left(M \alpha_{1} / 2 n^{2}\right) \underline{e}_{1}+\cdots+\left(M \alpha_{m} / 2 n^{2}\right) \underline{e}_{m}=\left(M / n^{2}, 0, \ldots, 0\right) .
$$

Thus, if we translate $S / 64$ successively by the vectors $\left(M \alpha_{j} / 2 n^{2}\right) \underline{e}_{j}, j=1, \ldots, m$, then we obtain the pyramid $S / 64+\left(M / n^{2}\right) \underline{v}$, and during these translations we never leave $S$.

For simpler notation we shift everything by $-\left(M / n^{2}\right) \underline{v}$. Under this shift $(S / 64)+\left(M / n^{2}\right) \underline{v}$ becomes $S / 64, S$ becomes $S^{*}:=S-\left(M / n^{2}\right) \underline{v}$ and $S / 128$ becomes $S^{* *}:=(S / 128)-\left(M / n^{2}\right) \underline{v}$ (see Figure 4$)$.

What we have just established can also be formulated in the following way: if we translate $S / 64$ successively by the vectors $-\left(M \alpha_{j} / 2 n^{2}\right) \underline{e}_{j}, j=m, \ldots, 1$, then we obtain a pyramid (which is twice the size of $S^{* *}$ ) with apex at the common apex of $S^{*}$ and $S^{* *}$, and during these translations we never leave $S^{*}$.

If we also define $F(x)=f\left(x+\left(M / n^{2}\right) \underline{v}\right)$ on $S^{*}$ and $P_{n}(x)=p_{n}\left(x+\left(M / n^{2}\right) \underline{v}\right)$, then (4.12) is the same as

$$
\left\|F-P_{n}\right\|_{S / 64} \leq C_{0} \omega_{S^{*}}^{r}(F, 1 / n)_{\mathcal{E}},
$$

and from here we want to conclude (4.13), which is the same as

$$
\left\|F-P_{n}\right\|_{S^{* *}} \leq C_{1} \omega_{S^{*}}^{r}(F, 1 / n)_{\mathcal{E}} .
$$

It will be proven in Lemma 7.3 (see also [14, Proposition 9.1]) that if $L>0$, then for any function $g \in C([0,1])$ and for any polynomial $q_{n}$ of a single variable 
and of degree at most $n$ with $n^{2} \geq 2 L$, we have

$$
\left\|g-q_{n}\right\|_{[0,1]} \leq C\left(\left\|g-q_{n}\right\|_{\left[L / n^{2}, 1\right]}+\omega_{[0,1]}^{r}(g, 1 / n)\right)
$$

where $C$ depends only on $L$ and $r$. This statement implies that a given rate of Jackson-type polynomial approximation, say

$$
\left\|g-q_{n}\right\|_{[0,1]} \leq C_{0} \omega_{\left[0,1+D / n^{2}\right]}^{r}(g, 1 / n)
$$

on the interval $[0,1]$ automatically holds on a $1+D / n^{2}$-dilation of the interval, i.e.

$$
\left\|g-q_{n}\right\|_{\left[0,1+D / n^{2}\right]} \leq D_{1} C_{0} \omega_{\left[0,1+D / n^{2}\right]}^{r}(g, 1 / n),
$$

with some $D_{1}$ depending on $D$, provided in the Jackson estimate (4.18) the modulus of smoothness is measured on the larger interval as is indicated in (4.18). On applying a linear transformation from $[0,1]$ to a segment, we get from (4.15) that if $I=[A, B]$ is a segment in $S / 64$ parallel to one of the apex edges of $S$ and $I^{\prime}:=\Phi_{A, 1+D / n^{2}} I$ is its dilation from $A$ with factor $1+D / n^{2}$, then we have

$$
\left\|F-P_{n}\right\|_{I^{\prime} \cap S^{*}} \leq D_{1} C_{0} \omega_{S^{*}}^{r}(f, 1 / n)_{\mathcal{E}},
$$

and here $D_{1}$ depends only on $D$. This allows us to extend the rate of approximation on $S / 64$ given in (4.15) to points lying outside $S / 64$. The set $S^{* *} \backslash(S / 64)$ in (4.16) consists of points lying close to $S / 64$ (they are of distance $O\left(1 / n^{2}\right.$ ) from $S / 64$ ), and we are going to use the just described extension process to cover these points. However, in this extension we must use the directions of the apex edges, and this creates problem, since not every point of $S^{* *}$ is directly reachable from $S / 64$ using segments in the apex edge directions (for example, the apex of $S^{* *}$ cannot be reached). Therefore, we shall need to use repeated extensions in different directions.

We shall use the product sets from Proposition 4.1. Let us denote $S / 64$ by $S^{\prime}$ and its base by $S_{0}^{\prime}$. If $Q_{1}, \ldots, Q_{m}$ are the vertices of $S_{0}$ (the base of $S$ ), then $Q_{j}^{\prime}=Q_{j} / 64$ are the vertices of $S_{0}^{\prime}=S_{0} / 64$. Let $1>\lambda>d /(d+1)$ be fixed so that $\lambda^{m}>1 / 2$. With an edge $e_{j}^{\prime}$ that connects the corresponding vertices of $S_{0}^{\prime}$ and $\lambda S_{0}^{\prime}$ consider the product set $\left(\lambda S_{0}^{\prime}\right) \times e_{j}^{\prime}$ (so $e_{j}^{\prime}$ is in the direction of the apex edge $\underline{e}_{j}$ and its length is $\left.(1-\lambda)\left|\underline{e}_{j}\right| / 64\right)$. This is part of $S^{\prime}$ (see Proposition 4.1 ), which means that from every point $A$ of $\Phi_{Q_{j}^{\prime}, \lambda} S_{0}^{\prime}$ we can draw a segment $I_{A}$ parallel with $\underline{e}_{j}^{\prime}$ to a boundary point $B$ of $S^{\prime}$, and the length of this segment is $\geq\left|e_{j}^{\prime}\right| \geq(1-\lambda)\left|e_{j}\right| / 64 \geq(1-\lambda) / 32$ (because $\underline{e}_{j}$ points from the origin to a point of the hyperplane $x_{1}=2$, so its length is at least 2). Since $I_{A}$ lies within $S^{\prime}$, we can use (4.19) and conclude that if $I_{A}^{\prime}$ is the dilation of $I_{A}$ from $A$ by a factor $1+D / n^{2}$, then on $I_{A}^{\prime} \cap S^{*}$ we have the bound

$$
\left\|F-P_{n}\right\|_{I_{A}^{\prime} \cap S^{*}} \leq D_{2} C_{0} \omega_{S^{*}}^{r}(f, 1 / n)_{\mathcal{E}}
$$

where $D_{2}$ depends only on $D$.

Since $\left|e_{j}^{\prime}\right| \geq(1-\lambda) / 32$, it is immediate that all these $I_{A}^{\prime}, A \in \Phi_{Q_{j}^{\prime}, \lambda} S_{0}^{\prime}$ cover the translation of $\lambda S^{\prime}$ by the vector $-\left(D(1-\lambda) / 32 n^{2}\right) \underline{e}_{j} /\left|\underline{e}_{j}\right|$ and for 
sufficiently large $D$ (depending on $\lambda$ and $M$ ), this will cover the translation of $\lambda S^{\prime}$ by the vector $-\left(M \alpha_{j} / 2 n^{2}\right) \underline{e}_{j}$ from (4.14). In later steps we shall use smaller copies (namely $\lambda^{k} S^{\prime}, k=1, \ldots, m$ ) of $S^{\prime}$ instead of $S^{\prime}$ itself, and for them the corresponding translation vectors will be $-\left(D(1-\lambda) \lambda^{k} / 32 n^{2}\right) \underline{e}_{j} /\left|\underline{e}_{j}\right|$, but for sufficiently large $D$ (depending on $\lambda$ and $M$ ), this is still at least as long as $-\left(M \alpha_{j} / 2 n^{2}\right) \underline{e}_{j}$ from (4.14) because we selected $\lambda$ so that $\lambda^{m} \geq 1 / 2$.

We apply this first with $j=m$, and recall that we said in connection with (4.14) that the translation $S^{\prime}-\left(M \alpha_{m} / 2 n^{2}\right) \underline{e}_{m}$ lies inside $S^{*}$, so (4.19) yields

$$
\left\|F-P_{n}\right\|_{\lambda S^{\prime}-\left(M \alpha_{m} / 2 n^{2}\right) \underline{e}_{m}} \leq D_{2} C_{0} \omega_{S^{*}}^{r}(f, 1 / n)_{\mathcal{E}} .
$$

Let us denote the transformation from $S^{\prime}$ to $\lambda S^{\prime}-\left(M \alpha_{j} / 2 n^{2}\right) \underline{e}_{j}$ by $\Psi_{j}\left(S^{\prime}\right)$. What we got can be written as

$$
\left\|F-P_{n}\right\|_{\Psi_{m}\left(S^{\prime}\right)} \leq D_{2} C_{0} \omega_{S^{*}}^{r}(f, 1 / n)_{\mathcal{E}} .
$$

Note that here $\Psi_{m}\left(S^{\prime}\right)$ is a $\lambda$-size copy of $S^{\prime}$ with apex at $-\left(M \alpha_{m} / 2 n^{2}\right) \underline{e}_{m}$. Now apply the same procedure to $\Psi_{m}\left(S^{\prime}\right)$, but with the edge $\underline{e}_{m}$ replaced by $\underline{e}_{m-1}$, to conclude

$$
\left\|F-P_{n}\right\|_{\Psi_{m-1}\left(\Psi_{m}\left(S^{\prime}\right)\right)} \leq D_{2}^{2} C_{0} \omega_{S^{*}}^{r}(f, 1 / n)_{\mathcal{E}},
$$

and here $\Psi_{m-1}\left(\Psi_{m}\left(S^{\prime}\right)\right)$ is a $\lambda^{2}$-size copy of $S^{\prime}$ with apex at $-\left(M \alpha_{m} / 2 n^{2}\right) \underline{e}_{m}-$ $\left(M \alpha_{m-1} / 2 n^{2}\right) \underline{e}_{m-1}$. Iterate this further with the edge directions $\underline{e}_{m-2}, \ldots, \underline{e}_{1}$. After $m$ iterations we obtain for $H=\Psi_{1}\left(\cdots\left(\Psi_{m}\left(S^{\prime}\right) \cdots\right)\right.$

$$
\left\|F-P_{n}\right\|_{H} \leq D_{2}^{m} C_{0} \omega_{S^{*}}^{r}(f, 1 / n)_{\mathcal{E}},
$$

and here $H$ is a $\lambda^{m}$-size copy of $S^{\prime}$ with apex at

$$
-\left(M \alpha_{m} / 2 n^{2}\right) \underline{e}_{m}-\left(M \alpha_{m-1} / 2 n^{2}\right) \underline{e}_{m-1}-\cdots-\left(M \alpha_{1} / 2 n^{2}\right) \underline{e}_{1} .
$$

In view of (4.14) this last point is precisely the apex of $S^{*}$, so $H$ is a homothetic copy of $S^{*}$ of size $\lambda^{m} / 64$ (recall that $S^{\prime}$ was a $1 / 64$ size copy of $S$ ) placed so that its apex coincides with the apex $\left(-M / n^{2}, 0, \ldots, 0\right)$ of $S^{*}$. Since $\lambda^{m} \geq 1 / 2$, this $H$ then covers $S^{* *}=S / 128+\left(-M / n^{2}, 0, \ldots, 0\right)$, hence (4.16) is a consequence of (4.21).

In the next section we use the following modification of Proposition 4.6.

Corollary 4.7 Let $\mathcal{E}^{*}$ be any set of directions that include the apex edge directions of $S$ and let $S \subset K$. Then for any fixed $M$

$$
E_{n}^{L}(f)_{S+\left(M / n^{2}\right) \underline{v}} \prec \omega_{K}^{r}(f, 1 / n)_{\mathcal{E}^{*}}
$$

implies

$$
E_{n}^{L}(f)_{S / 2} \prec \omega_{K}^{r}(f, 1 / n)_{\mathcal{E}^{*}} .
$$

Furthermore, in this conclusion $\underline{v}$ can be any fixed vector pointing from the apex of $S$ to an inner point of $S$. 
The proof of this is the same that was given for Proposition 4.6 (just replace in that proof $S / 64$ by $S$ ), since in that proof we only used apex edge directions of $S$ and the fact that the vector $\underline{v}$ was a convex combination of the corresponding apex edge vectors $\left(\underline{e}_{j}\right.$ in the proof of Proposition 4.6).

\section{Small degrees}

In this section we show that the Jackson type estimate that we are discussing on convex polytopes holds for every fixed $n \geq r d$ (by a bounded linear operator), so in the previous part of the paper we could concentrate only on large degree polynomial approximation.

Theorem 5.1 If $K$ is a convex polytope in $\mathbf{R}^{d}$, then for every $n \geq$ rd there is a bounded linear operator $L_{n}: C(K) \rightarrow \Pi_{n}^{d}$ and a constant $C$ independent of $f$ such that

$$
\left\|f-L_{n} f\right\|_{K} \leq C \omega_{K}^{r}(f, 1 / n) .
$$

Here $C$ may depend on $n$ (and of course, on $K$ ).

Proof. Take a vertex of $K$ and $d+1$ independent edge directions $\underline{e}_{1}, \ldots, \underline{e}_{d+1}$ at this vertex. With these directions form a parallelepiped $H \subset K$ as its edge directions inside $K$. We may assume that the origin is in the interior of $H$, and let $\theta K$ be a dilation of $K$ from the origin with some small dilation factor $\theta$ such that $\theta K$ lies in the interior of $H$.

The claim is true on parallelepipeds, see [6, Theorem 12.1.1] (there the degree of a polynomial was the maximal degree in each variables, and the result was for all polynomials with that degree $\geq r$; but now we use total degree, so we can claim the result for parallelepipeds for polynomials of total degree $\geq r d$ ). Thus, we have for some $L_{n}$

$$
\left\|f-L_{n} f\right\|_{H} \leq C_{0} \omega_{H}^{r}(f, 1 / n) \leq C_{0} \omega_{K}^{r}(f, 1 / n),
$$

hence

$$
\left\|f-L_{n} f\right\|_{\theta K} \leq C_{0} \omega_{K}^{r}(f, 1 / n),
$$

is even more true. Thus, all we need to show is that (5.1) is a consequence of (5.2).

For simpler notations we write $K$ instead of $\theta K$ and $(1 / \theta) K$ instead of $K$, and what we are going to prove is that if $P_{n}$ is any polynomial of the fixed degree $n$ and

$$
\left\|f-P_{n}\right\|_{K} \leq C_{0} \omega_{K / \theta}^{r}(f, 1 / n),
$$

then

$$
\left\|f-P_{n}\right\|_{K / \theta} \leq C_{1} \omega_{K / \theta}^{r}(f, 1 / n) .
$$

To this end it is sufficient to show that there is a $1<\lambda_{0}<1 / \theta$ depending on $K$ such that for every $1 \leq \lambda \leq \lambda_{0}$

$$
\left\|f-P_{n}\right\|_{K} \leq C_{0} \omega_{\lambda K}^{r}(f, 1 / n)
$$


implies

$$
\left\|f-P_{n}\right\|_{\lambda K} \leq C_{2} \omega_{\lambda K}^{r}(f, 1 / n) .
$$

Indeed, then choose such a $\lambda$ with $\lambda^{k}=1 / \theta$ with some $k$, and iterate (5.5) $\Rightarrow(5.6) k$-times (with $K$ replaced by $\lambda^{l} K, l=0,1, \ldots, k-1$, successively) to obtain (5.4). (The iteration is legitimate because any $\lambda^{l} K$ is a homothetic copy of $K$ and $\omega_{\lambda^{l} K}(f, 1 / n) \leq \omega_{K / \theta}(f, 1 / n)$ for $\left.l \leq k\right)$.

For the implication (5.5) $\Rightarrow(5.6)$ we have done most of the work in the proof of Proposition 4.6. Let $S$ be a small pyramid with apex at a point $Q$ such that the apex edge directions of $S$ are among the edge directions of $K$, and let $S_{\eta}$ be the $\eta$-dilation of $S$ from its apex. Let also $\tilde{S}_{6 / 7}$ be a translation of $S_{6 / 7}$ so that it lies in the interior of $S$. In the proof of Proposition 4.6 and Corollary 4.7 we verified that if $\tilde{K}$ is a polytope such that $S \subset \tilde{K}$ and the apex edge directions of $S$ are among the edge directions of $\tilde{K}$, then

$$
\left\|f-P_{n}\right\|_{\tilde{S}_{6 / 7}} \leq C_{0} \omega_{\tilde{K}}^{r}(f, 1 / n)
$$

implies

$$
\left\|f-P_{n}\right\|_{S_{3 / 7}} \leq C_{1} \omega_{\tilde{K}}^{r}(f, 1 / n)
$$

with some $C_{1}$ that depends on $C_{0}$ and $n$. In fact, in that proof we worked with translations of size $1+D / n^{2}$, but if $n$ is fixed, then this can be of any size if $D$ is sufficiently large. So Proposition 4.6 and its proof gives, indeed, the implication $(5.7) \Rightarrow(5.8)$.

Take now an $\eta$-pyramidal covering of $K$ (with some small but fixed $\eta$ ) as in Section 3. Recall that this was a covering by some pyramids $\mathcal{K}_{\eta / 2, s}$, where $\mathcal{K}_{\eta / 2, s}$ is a $1 / 2$-scaled version of a larger pyramid $K_{\eta, s}$ (still lying in $K$ ) scaled from its apex. Fix for each $K_{\eta, s}$ a vector $\underline{u}_{s}$ from its apex pointing to an inner point of $K_{\eta, s}$. Let $\lambda>1$. Then $\left\{\lambda \mathcal{K}_{\eta / 2, s}\right\}$ is an $\eta$-pyramidal covering of $\lambda K$, and from the construction of $\eta$-pyramidal covering in Section 3 it follows that if $\lambda>1$ is sufficiently close to 1 , then each $\lambda \mathcal{K}_{\eta / 2, s}+\underline{u}_{s}$ lies in $K$. Imagine now that the above $\tilde{S}_{6 / 7}$ is one of these $\lambda K_{\eta, s}+\underline{u}_{s}$ and $S_{3 / 7}$ is precisely $\lambda \mathcal{K}_{\eta / 2, s}$. (5.7) for $\tilde{K}=\lambda K$ is then a consequence of (5.5), and in the conclusion (5.8) the right hand side is at most as large as $C_{1} \omega_{\tilde{K}}^{r}(f, 1 / n)=C_{1} \omega_{\lambda K}^{r}(f, 1 / n)$, so

$$
\left\|f-P_{n}\right\|_{\lambda \mathcal{K}_{\eta / 2, s}} \leq C_{1} \omega_{\lambda K}^{r}(f, 1 / n)
$$

follows. Since the sets $\lambda \mathcal{K}_{\eta / 2, s}$ for all $s$ cover $\lambda K$, we obtain (5.6).

This completes the proof of the implication (5.5) $\Rightarrow(5.6)$ for all $\lambda>1$ lying sufficiently close to 1 , and that finishes the proof of Theorem 5.1. 


\section{Part II}

\section{Approximation on algebraic domains}

In the second part of this paper we shall deal with domains with smooth (actually algebraic) boundary - see Definition 6.3 for the precise concept. So far in the literature the rate of approximation has been characterized in details via appropriate moduli of smoothness only on balls, see [3] and [4] by F. Dai and Y. $\mathrm{Xu}$. Balls exhibit a large degree of symmetry, and in this paper we shall break this symmetry, and prove the characterization for a large family of domains.

\section{Domains and moduli of smoothness}

In this section we shall be primarily concerned how to define appropriate moduli of continuity/smoothness on smooth domains and how the geometry influences the construction.

\subsection{Domains in $\mathbf{R}^{2}$}

We start with two-dimensional domains. Let $G \subset \mathbf{R}^{2}$ be the closure of a bounded, finitely connected domain (connected open set) with $C^{2}$ boundary $\Gamma$. For simpler discussion we shall sometimes identify $\mathbf{R}^{2}$ with the complex plane. Since $\Gamma$ is $C^{2}$ smooth, there is an $r_{0}$ such that a circle of radius $8 r_{0}$ can be drawn (from the inside) to every point of $\Gamma$ which stays inside $G$ together with its interior.

The modulus of smoothness on a circle $C_{\rho}\left(z_{0}\right) \subset G$ about a center $z_{0}$ and of radius $\rho$ is defined as in classical trigonometric approximation theory, namely

$$
\omega_{C_{\rho}\left(z_{0}\right)}^{1}(f, \delta):=\sup _{\theta \leq \delta, \varphi}\left|f\left(z_{0}+\rho e^{i(\varphi+\theta / 2)}\right)-f\left(z_{0}+\rho e^{i \varphi-\theta / 2}\right)\right|
$$

(here and in what follows in such expressions, $\theta \leq \delta$ means that $0 \leq \theta \leq \delta$ ), and the higher order moduli of smoothness as

$$
\begin{aligned}
\omega_{C_{\rho}\left(z_{0}\right)}^{r}(f, \delta) & :=\sup _{\theta \leq \delta, \varphi}\left|\Delta_{\theta}^{r} f\left(z_{0}+\rho e^{i(\varphi+\cdot)}\right)\right| \\
& =\sup _{\theta \leq \delta, \varphi}\left|\sum_{j=0}^{r}(-1)^{j}\left(\begin{array}{l}
r \\
j
\end{array}\right) f\left(z_{0}+\rho e^{i(\varphi+(r / 2-j) \theta)}\right)\right| .
\end{aligned}
$$

If $I=\overline{A B} \subset G$ is a segment, then the modulus of smoothness is defined on $I$ as in (1.6):

$$
\omega_{I}^{r}(f, \delta)=\sup _{h \leq \delta, z \in I} \| \Delta_{h \tilde{d}_{I}(\underline{e}, z) \underline{e}}^{r} f(z) \mid
$$




$$
=\sup _{h \leq \delta, z \in I}\left|\sum_{j=0}^{r}(-1)^{j}\left(\begin{array}{l}
r \\
j
\end{array}\right) f\left(z+(r / 2-j) h \tilde{d}_{I}(\underline{e}, z)\right)\right|,
$$

where $\underline{e}$ is the direction of $I=\overline{A B}$ and $\tilde{d}_{I}(\underline{e}, z)=\sqrt{|z-A||z-B|}$. Recall the usual convention that here $\Delta_{h \tilde{d}_{I}(\underline{e}, z) \underline{e}}^{r} f(z)$ is defined to be 0 if either of the points $z \pm(r / 2) h \tilde{d}_{I}(\underline{e}, z)$ does not belong to $I$. Finally, we set

$$
\omega^{r}(f, \delta)_{G}=\max \left(\sup _{C_{\rho}\left(z_{0}\right)} \omega_{C_{\rho}\left(z_{0}\right)}^{r}(f, \delta), \sup _{I} \omega_{I}^{r}(f, \delta)\right),
$$

where the suprema are taken for all circles $C_{\rho}\left(z_{0}\right)$ that lie in $G$, and for all horizontal or vertical segments $I \subset G$.

We shall not deal with the various properties of these moduli, but we shall frequently use that

$$
\omega^{r}(f, \lambda \delta)_{G} \leq C \lambda^{r} \omega^{r}(f, \delta)_{G}, \quad \lambda \geq 1 .
$$

Indeed, this inequality is well known (see [5, Chapter 2, (7.8)] and [6, Theorem 4.1.2]) for both $\omega_{I}^{r}$ and $\omega_{C_{\rho}\left(z_{0}\right)}^{r}$, from which the claim follows.

We shall also often use that if $G_{1} \subset G$, then $\omega^{r}(f, \delta)_{G_{1}} \leq \omega^{r}(f, \delta)_{G}$, which is clear from the definition.

Remark 6.1 1. One could request the second supremum in (6.4) only for (horizontal or vertical) maximal segments $I$ that are contained in $G$, the value of $\omega^{r}(f, \delta)_{G}$ would not change.

2. In (6.4) the supremum is take for all circles $C_{\rho}\left(z_{0}\right)$ that lie in $G$. For first order, i.e. for modulus of continuity, it would be sufficient to take the supremum in (6.4) only for circles $C_{\rho}$ of the fixed radius $\rho=r_{0}$, but it is not clear if that is sufficient when proving Jackson type theorems via higher order moduli. A careful examination of the proof of Theorem 6.5 below shows, however, that in the case of $r$-th order modulus of smoothness there is a $\rho_{0} \leq r_{0}$ such that it is sufficient to take the supremum in (6.4) only for circles $C_{\rho}$ lying in $G$ with $\rho_{0} / 2 \leq \rho \leq \rho_{0}$.

3. The way they were defined, the moduli of continuity/smoothness in (6.4) depend on the position of $G$ in the coordinate system (since horizontal/vertical segments are used in them). If the second supremum in (6.4) is taken for all segments in $G$, then the so obtained (larger) moduli would already be isometry invariant, and everything in what follows would work for these modified moduli exactly as for the $\omega^{r}(f, \delta)_{G}$.

4. On domains in $\mathbf{R}^{2}$ for a different modulus of smoothness based on averages see the paper [8] by K. G. Ivanov. With that modulus the correct order Jackson theorem was announced in [8] for domains with piecewise $C^{2}$ boundary, as well a corresponding weak converse in some special cases. One should also mention the works [7] and [9] that contain the first results on approximation on domains by polynomials in several variables. 


\subsection{Domains in $\mathbf{R}^{d}$}

Now let $G \subset \mathbf{R}^{d}$ be the closure of a bounded, finitely connected domain (in short "closed domain") with $C^{2}$ boundary $\Gamma$. Since the boundary $\Gamma$ is $C^{2}$ smooth, there is an $r_{0}$ such that a $(d-1)$-dimensional sphere of radius $4 d r_{0}$ can be drawn (from the inside) to every point of $\Gamma$ which stays inside $G$ together with its interior.

We say that a circle $C_{\rho}$ is parallel with a coordinate plane if its plane is parallel with one of the planes spanned by two of the coordinate axes. The modulus of smoothness on such a circle $C_{\rho} \subset G$ is defined as before in (6.2), and the modulus of continuity on a segment $I \subset G$ as in (6.3). Finally let

$$
\omega^{r}(f, \delta)_{G}=\max \left(\sup _{C_{\rho}} \omega_{C_{\rho}}^{r}(f, \delta), \sup _{I} \omega_{I}^{r}(f, \delta)\right),
$$

where the suprema are taken for all circles $C_{\rho} \subset G$ (of arbitrary radius $\rho>0$ ) which are parallel with a coordinate plane and for all segments $I \subset G$ that are parallel with one of the coordinate axes.

Remark 6.2 Saying that $C_{\rho}$ is parallel with a coordinate plane and using the modulus of smoothness on $C_{\rho}$ is pretty much the same as using the so called Euler angles as in [3], [4], so our $\omega^{r}(f, \delta)_{G}$ is close to the moduli of smoothness used on the unit ball in $\mathbf{R}^{d}$ in those works.

We shall work with domains in $\mathbf{R}^{d}$ bounded by algebraic surfaces. So let $\Gamma_{1}, \ldots, \Gamma_{k}$ be the connected components of $\Gamma=\partial G$, and we assume that there are polynomials $\Phi_{j}\left(x_{1}, \ldots, x_{d}\right)$ of $d$ variables such that $\Gamma_{j}$ is part of the surface $\Phi_{j}\left(x_{1}, \ldots, x_{d}\right)=0$. We shall also assume that on $\Gamma_{j}$ the gradient $\nabla \Phi_{j}$ does not vanish. Then this gradient is a normal to $\Gamma_{j}$.

Definition 6.3 We say that $G$ is an algebraic domain if all these properties are satisfied.

Examples include balls, domains enclosed by thori, etc., but there are many more exotic examples like the one ${ }^{4}$ in Figure 5. Note also that if $G$ is as explained and we remove from the interior of $G$ disjoint simply connected domains $G_{1}, \ldots, G_{k}$ satisfying similar conditions, then $G \backslash\left(\operatorname{Int}\left(G_{1}\right) \cup \cdots \cup \operatorname{Int}\left(G_{k}\right)\right)$ also satisfies the conditions set forth.

Remark 6.4 We emphasized that we do not assume that $\Gamma_{j}$ is the whole surface $\Phi_{j}\left(x_{1}, \ldots, x_{d}\right)=0$, it can be just as well one of its components.

\subsection{The Jackson theorem and its converse}

Let $G$ be a closed domain as described before and let

$$
E_{n}(f)_{G}=\inf _{P_{n} \in \Pi_{n}^{d}}\left\|f-P_{n}\right\|_{G}
$$

\footnotetext{
${ }^{4}$ Courtesy of János Karsai, who used Mathematica 11.3 to create the figure
} 


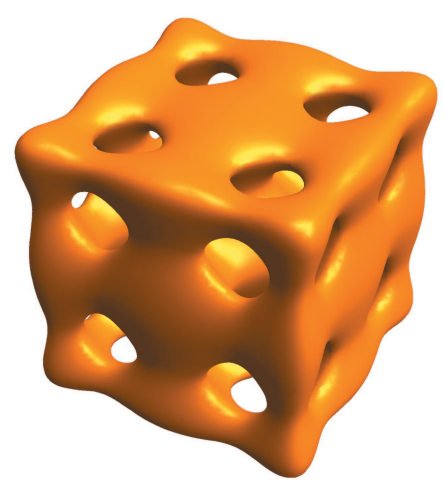

Figure 5: The domain enclosed by the so called Banchoff surface $x^{2}\left(3-4 x^{2}\right)^{2}+$ $y^{2}\left(3-4 y^{2}\right)^{2}+z^{2}\left(3-4 z^{2}\right)^{2}=3 / 2$

be the error of best polynomial approximation to $f \in C(G)$ by polynomials of $d$-variables of total degree at most $n$. We have the following Jackson theorem ${ }^{5}$

Theorem 6.5 If $G$ is a (closed) algebraic domain, then for $r=1,2, \ldots$

$$
E_{n}(f)_{G} \leq C \omega^{r}(f, 1 / n)_{G}, \quad n \geq r d,
$$

with a constant $C$ independent of $f$ and $n$.

The standard weak converse is true:

Theorem 6.6 With the assumptions of Theorem 6.5

$$
\omega^{r}(f, 1 / n)_{G} \leq \frac{C}{n^{r}} \sum_{k=0}^{n}(k+1)^{r-1} E_{k}(f)_{G},
$$

with a constant $C$ independent of $n \geq r d$.

Since the restriction of a polynomial $P_{n}\left(x_{1}, \ldots, x_{d}\right)$ to any line segment is an algebraic polynomial of a single variable, and its restriction to any circle $C_{\rho}$, say to $C_{\rho}\left(z_{0}\right)=\left\{\left(x, y, \zeta_{2}, \ldots, \zeta_{d}\right)\right\}, z_{0}=\left(x_{0}, y_{0}, \zeta_{2}, \ldots, \zeta_{d}\right), x=x_{0}+\rho \cos \theta, y=$ $y_{0}+\rho \sin \theta, x_{3}=\zeta_{3}, \ldots, x_{d}=\zeta_{d}$, is a trigonometric polynomial of degree at most $n$ in $\theta$, the inequality (6.6) is an immediate consequence of the corresponding inequalities on $[-1,1]$ and on the unit circle (see $[6,7.2 .4]$ and $[5$, Theorem 7.3.1]) if we take into account the definition of the modulus of smoothness $\omega_{G}^{r}$. Therefore, in what follows we shall only concentrate on (6.5).

We also state

\footnotetext{
${ }^{5}$ See also the note at the end of the paper
} 
Corollary 6.7 Let $0<\alpha$. If $f \in C(G)$ can be approximated with error $\leq 1 / n^{\alpha}$ by algebraic polynomials of degree at most $n$ on every segment of $G$ parallel to one of the coordinate axes, and on every circle lying in $G$ parallel with one of the coordinate planes, then $f$, on the whole of $G$, can be approximated with error $\leq C / n^{\alpha}$ by polynomials of d-variables of degree at most $n$.

This corollary says that in certain cases $d$-variable polynomial approximation can be reduced to ordinary one-variable trigonometric and algebraic approximation.

The major steps in the proof of Theorem 6.5 are as follows. First of all, in the next section it is shown that all difficulties arise around the boundary of the domain: we shall decompose a relative neighborhood of this boundary into small pieces, and if approximation can be done in the correct order on the individual pieces, then the global result follows. The different pieces can be handled the same way, so the problem will be to prove the correct rate of approximation on a small part around the boundary. This small part is the image of a cube under a polynomial mapping, and the problem will be transformed into a corresponding approximation problem on the cube. The case of a cube is well-known and classical, however, the transformation used is not linear, and a major effort will be to establish how the moduli of smoothness change under the given transformation. This is a highly non-trivial problem (to be done in Sections 8 and 10) because the distance from the boundary plays an important role in the definition of the moduli of smoothness. The $r=1$ case shows all the difficulties coming from the geometry and of the just mentioned transformation, and this case will be dealt with separately in Section 8. The general $r>1$ case will involve estimates for derivatives of composed functions (detailed in Section 10), as well as the special case when the domain is a disk (detailed in Section 9). This approach gives the required estimate for the moduli of smoothness of the transformed function on the whole domain except for points that lie too close to the boundary (closer than $C / n^{2}$ for $n$-th degree approximation). Finally, Section 11 establishes that that is sufficient to have the same estimate on the whole domain, and that section contains also the completion of the proof.

\section{Reduction to approximation close to the bound- ary}

In this section we start the proof of Theorem 6.5. We show that if we can prove a Jackson-type bound on suitable sets attached to the boundary (with the modulus of smoothness on the whole domain), then Theorem 6.5 follows.

The Jackson-type estimate (6.5) can be proven for each fixed $n$ by a constant that may depend on $n$ (we do not give details, they are similar - actually simpler - to what was used in Section 5), so in what follows we may concentrate only on approximation for large $n$. 
Let $\underline{e}_{1}, \ldots, \underline{e}_{d}$ be the unit vectors in $\mathbf{R}^{d}$ pointing to the positive directions of the corresponding coordinate axis. If $P$ is a point on $\Gamma$ and $\underline{n}_{P}$ is the unit inner normal to $\Gamma$ at $P$, then there is a $j$ for which the dot product $\left|\underline{n}_{P} \cdot \underline{e}_{j}\right|$ is maximal. Then we must have $\left|\underline{n}_{P} \cdot \underline{e}_{j}\right| \geq 1 / \sqrt{d}$ (the square of the left hand sides for all $j$ sums up to 1 ), and for all other $k$ the inequality $\left|\underline{n}_{P} \cdot \underline{e}_{k}\right| \leq 1 / \sqrt{2}$ is a consequence of the definition of $\underline{e}_{j}$. Then there is a small neighborhood of $P$ such that for all $Q \in \Gamma$ in that neighborhood we have $\left|\underline{n}_{Q} \cdot \underline{e}_{j}\right| \geq 1 / 2 \sqrt{d}$, and for all other $k$ the inequality $\left|\underline{n}_{Q} \cdot \underline{e}_{k}\right| \leq 3 / 4$ is true, and call the part of $\Gamma$ (more precisely the part of the connected component of $\Gamma$ that contains $P$ ) that lies in that neighborhood an $x_{j}$-surface. Since $x_{j}$-surfaces for different $j$ can be similarly handled, in what follows we shall assume that $j=d$, and that close to $P$ the domain $G$ lies above $\Gamma$ (in the direction of the $x_{d}$-axis). Around $P$ the boundary $\Gamma$ is part of a level surface $\Phi_{s}=0$, hence the normal vector to $\Gamma$ is just the normalized gradient (or its negative) $\nabla \Phi_{s}$. Therefore, $\left|\partial \Phi_{s} / \partial x_{d}\right| \geq c / 2 \sqrt{d}$ in the neighborhood in question with some $c>0$. Thus, by the implicit function theorem, there is a cube $I_{P} \subset \mathbf{R}^{d-1}$ such that in a neighborhood of $P$ the boundary $\Gamma$ is given by the graph of a function $F_{P}\left(x_{1}, \ldots, x_{d-1}\right),\left(x_{1}, \ldots, x_{d-1}\right) \in I_{P}$. Call this graph $\Gamma_{P}-$ it is part of $\Gamma$. By shrinking $I_{P}$ if necessary, we may assume that $P$ is the image of the center of $I_{P}$ under the mapping $F_{P}$. Since we are dealing with algebraic domains with nonzero gradient of the functions that define the boundary, it is easy to see that this $I_{P}$ can be chosen so that its diameter is bigger than a positive number independent of $P$ : $\operatorname{diam}\left(I_{P}\right) \geq \delta_{0}>0$.

Consider the set

$$
\begin{aligned}
G_{P}= & \left\{\left(x_{1}, \ldots, x_{d}\right) \mid\left(x_{1}, \ldots, x_{d-1}\right) \in I_{P},\right. \\
& \left.x_{d} \in\left[F_{P}\left(x_{1}, \ldots, x_{d-1}\right), F_{P}\left(x_{1}, \ldots, x_{d-1}\right)+r_{0}\right]\right\}
\end{aligned}
$$

(recall the definition of $r_{0}$ from Section 6), i.e. we place a segment of length $r_{0}$ parallel with the $x_{d}$-axis "above" every point $Q \in \Gamma_{P}$, see Figure 6 . For a $Q \in \Gamma_{P}$ let $S_{Q}$ be the sphere of radius $4 d r_{0}$ which lies on the side of $\Gamma_{P}$ as $G$ (in a small neighborhood of $\Gamma_{P}$ ) and touches $\Gamma$ at the point $Q$ (i.e. $S_{Q}$ goes through $Q$ and at $Q$ it has the same tangent hyperplane as $\Gamma$ ). Using the fact that $S_{Q}$ lies inside $G$ together with its interior (this follows from the definition of $r_{0}$ ), we can conclude that $G_{P}$ is a subset of $G$. Indeed, the condition $\left|n_{Q} \cdot \underline{e}_{d}\right| \geq 1 / 2 \sqrt{d}$ is the same as saying that the projection of the segment in between $Q$ and the center of $S_{Q}$ onto the line through $Q$ in the $\underline{e}_{d}$ direction is at least $4 d r_{0} / 2 \sqrt{d}>2 r_{0}$, so the $\underline{e}_{d}$ directional segment of length $r_{0}$ placed to $Q$ lies inside the sphere $S_{Q}$.

We claim that $f$ can be approximated on $G_{P}$ in the order $\omega^{r}(f, 1 / n)_{G}$ by polynomials of $d$ variables of degree at most $n$, i.e.

$$
E_{n}(f)_{G_{P}} \leq C \omega^{r}(f, 1 / n)_{G}
$$

This is the hard part of the proof, and in this section we only show how the proof of Theorem 6.5 can be completed from (7.2).

Let $I_{P}^{*}$ be the dilation of $I_{P}$ from its center by a factor $1 / 2$, and let $\Gamma_{P}^{*}$ be graph of the function $\Gamma\left(x_{1}, \ldots, x_{d-1}\right)$ over $I_{P}^{*}$. Then $\Gamma_{P}^{*}$ is part of $\Gamma_{P}$.. Over $\Gamma_{P}^{*}$ 


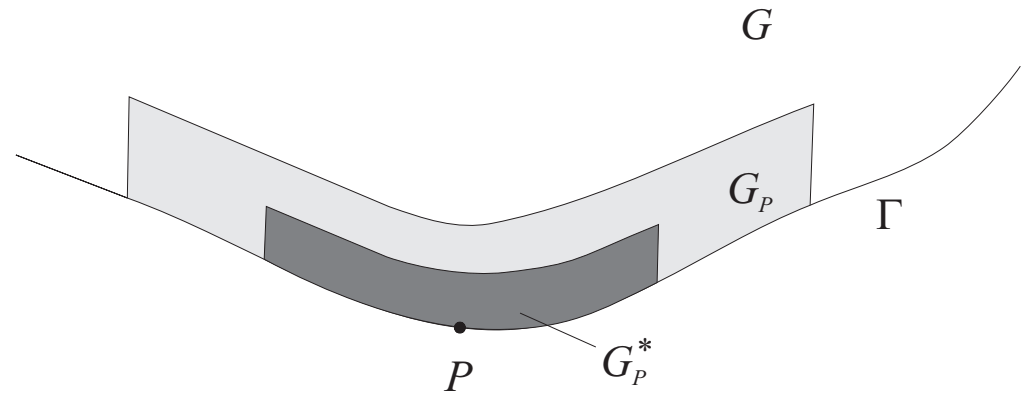

Figure 6: The domains $G, G_{P}$ and $G_{P}^{*}$

form the corresponding set $G_{P}^{*}$, with the difference that now use $\underline{e}_{d}$-directional segments over the points of $\Gamma_{P}^{*}$ of length $r_{0} / 2$ (see Figure 6 ). Then $G_{P}$ contains a relative neighborhood of $G_{P}^{*}$ with respect to $G$. Since $\operatorname{diam}\left(I_{P}^{*}\right) \geq \delta_{0} / 2$ and the functions $F_{P}$ are uniformly $C^{2}$ (actually, $C^{\infty}$ ) smooth, there is a $\delta_{1}>0$ independent of $P \in \Gamma$ such that $G_{P}^{*}$ covers the relative $\delta_{1}$-neighborhood of $P$ (relative with respect to $G$ ), i.e. $B_{\delta_{1}}(P) \cap G \subseteq G_{P}^{*}$, where $B_{\delta}(P)$ is the ball of radius $\delta$ about $P$. Let $\left\{P_{i}\right\}_{i=1}^{k} \subset \Gamma$ be a finite set such that every point of $\Gamma$ is of distance $\leq \delta_{1} / 4$ from some $P_{i}$. It is clear that $\cup \Gamma_{P_{i}}^{*}$ covers $\Gamma$, furthermore $\cup_{i} B_{\delta_{1}}\left(P_{i}\right)$, and hence also $\cup_{i} G_{P_{i}}^{*}$, covers the (open) $\delta_{1} / 2$-neighborhood $O$ of $\Gamma$. Cover the rest of $G$ by the interiors of finitely many $d$-dimensional closed cubes $U_{i}^{*}, 1 \leq i \leq l$, so that if $U_{i}$ is the dilation of $U_{i}^{*}$ from its center by some factor $(1+\theta)$ with some small $\theta>0$, then even $U_{i}$ lies in $G$. We can choose these $U_{i}$ so that their edges are parallel with the coordinate axes. On each $U_{i}$ we have the analogue of (7.2):

$$
E_{n}(f)_{U_{i}} \leq C \omega^{r}(f, 1 / n)_{G} .
$$

Indeed, this follows from [6, Theorem 12.1.1] (or from Theorem 1.1 in the first part of this paper) and from how $\omega^{r}(f, 1 / n)_{G}$ has been defined. Since the interiors of $G_{P_{i}}^{*}, i=1,2, \ldots, k$ and of $U_{i}^{*}, i=1,2, \ldots, l$ cover the interior of $G$, we can invoke Lemma 3.9 and conclude that from (possibly repeated) copies of the $U_{i}^{*}$ and from (possibly repeated) copies of the sets $G_{P_{i}}^{*}$ we can form a sequence of sets $K_{s}^{*}, 1 \leq s \leq T$, and the corresponding sequence of sets $K_{s}$ (which is $U_{j}$ if $K_{s}^{*}=U_{j}^{*}$ and it is $G_{P_{j}}$ if $K_{s}^{*}=G_{P_{j}}^{*}$ ) that satisfy

(i) each $K_{s}$ is contained in $G$,

(ii) $G=\cup_{s=1}^{T} K_{s}^{*}$,

(iii) the intersection of the interiors of $K_{s}^{*}$ and $K_{s+1}^{*}$ is non-empty for all $s=$ $1, \ldots, T-1$

(iv) for some $\varepsilon>0$ we have $\left(K_{s}^{*}\right)^{\varepsilon} \cap G \subset K_{s}$, where $\left(K_{s}^{*}\right)^{\varepsilon}$ is the closed $\varepsilon$ neighborhood of $K_{s}^{*}$. 
Note that (iv) is the consequence of (i) and the fact that each $K_{s}$ contains a (relative to $G$ ) neighborhood of $K_{s}^{*}$.

This is now the analogue of the $\eta$-pyramid covering from Proposition 3.8, and note that on each $K_{s}$ we have

$$
E_{n}(f)_{K_{s}} \leq C \omega^{r}(f, 1 / n)_{G}
$$

by (7.2)-(7.3). So we can combine local approximants as in Section 3, and to that we shall only need the following analogue of Lemma 3.4.

Lemma 7.1 Let $S^{*}$ be one of the sets $K_{s}^{*}$ and $S$ the corresponding set $K_{s}$, and let $H \subset G$ be a closed set such that for some $\delta>0$ the intersection $S^{*} \cap H$ contains a ball of radius $\delta$. Then for every $l_{0}$ there is an $l$ such that

$$
E_{l_{0} n}(f)_{S} \prec \omega^{r}(f, 1 / n)_{G}, \quad E_{l_{0} n}(f)_{H} \prec \omega^{r}(f, 1 / n)_{G}, \quad f \in C(G)
$$

imply

$$
E_{l n}(f)_{S^{*} \cup H} \prec \omega^{r}(f, 1 / n)_{G}, \quad f \in C(G) .
$$

If we repeatedly apply this with $H=\cup_{j=1}^{s-1} K_{j}^{*}$ and $S^{*}=K_{s}^{*}, S=K_{s}$, then for $s=T$ we can conclude from (7.6) the Jackson estimate (6.5) for large $n$.

Proof of Lemma 7.1. We may assume that $G$ lies in the unit ball $B_{1}(0)$ of $\mathbf{R}^{d}$, and follow the proof of Lemma 3.4. It is clear that all we need to prove is the following variant of Lemma 3.2 .

Lemma 7.2 Let $S^{*}$ be one of the $K_{s}^{*}$ and $S$ the corresponding set $K_{s}$. There is a constant $k=k(S)$ such that for every $n \geq 1$ there are polynomials $R_{k n}$ of degree at most kn for which

a) $0 \leq R_{k n}(x) \leq 1$ for $x \in B_{1}(0)$,

b) $1-R_{k n}(x) \leq 2^{-n}$ for $x \in S^{*}$,

c) $R_{k n}(x) \leq 2^{-n}$ for $x \in G \backslash S$.

Proof. If $K_{s}$ is a cube $U_{i}$, then this follows from Lemma 3.2, so let $K_{s}$ be one of the sets $G_{P_{j}}$, say $G_{P}$.

Cover $S^{*}=G_{P}^{*}$ by cuboids $V_{1}^{*}, \ldots, V_{m}^{*}$ as depicted in Figure 7 so that for some $\varepsilon>0$ it is true that if $V_{j}$ is the $(1+\varepsilon)$-enlarged copy of $V_{j}^{*}$ enlarged from its center, then $V_{j}$ also lies in $B_{1}(0)$ and $V_{j} \cap G$ lies in $G_{P}$. By Lemma 3.2 for some $l$ and all $n \geq 1$ there are polynomials $r_{l n, j}$ of degree at most $\leq \ln$ that satisfy

1) $0 \leq r_{l n, j}(x) \leq 1$ for $x \in B_{1}(0)$,

2) $1-r_{l n, j}(x) \leq 3^{-n}$ for $x \in V_{j}^{*}$,

3) $r_{l n, j}(x) \leq 3^{-n}$ for $x \in B_{1}(0) \backslash V_{j}$. 


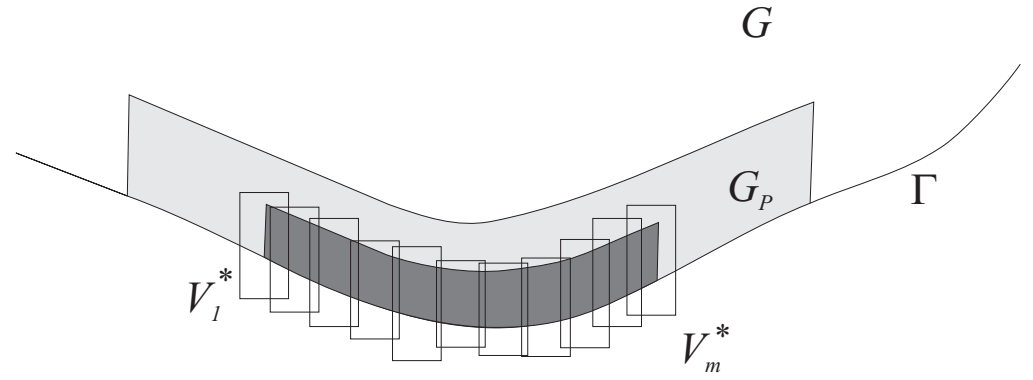

Figure 7: The sets $V_{j}^{*}$

With $P_{0} \equiv 0$, for $j=1, \ldots, m$ set $P_{j}(x)=P_{j-1}(x)\left(1-r_{l n, j}(x)\right)+r_{l n, j}(x)$. These are polynomials of degree at most $k n$ with $k=l m$, and induction on $j$ gives that
A) $0 \leq P_{j}(x) \leq 1$ for $x \in B_{1}(0)$,
B) $1-P_{j}(x) \leq 3^{-n}$ for $x \in \cup_{s=1}^{j} V_{s}^{*}$,
C) $P_{j}(x) \leq j 3^{-n}$ for $x \in B_{1}(0) \backslash \cup_{s=1}^{j} V_{s}$.

On applying this for $j=m$ we obtain a)-c) for all sufficiently large $n$ for the polynomials $R_{k n}(x):=P_{m}(x)$.

In some cases we cannot directly do the approximation up to the boundary, just if we stay off it at least $L / n^{2}$ distance (for $n$-th degree approximation). However, in such situations the missing range (closer than $L / n^{2}$ to the boundary) is automatically covered by the same approximants. This will follow from the next lemma.

Lemma 7.3 Let $g \in C[0,1]$ and let $L>1$ be fixed. Then for any polynomial $q_{n}$ of a single variable and of degree at most $n$ with $n^{2} \geq 2 L$, we have

$$
\left\|g-q_{n}\right\|_{[0,1]} \leq C\left(\left\|g-q_{n}\right\|_{\left[L / n^{2}, 1\right]}+\omega_{[0,1]}^{r}(g, 1 / n)\right),
$$

where $C$ depends only on $L$ and $r$.

Remark 7.4 The lemma implies for any fixed $0<a \leq 1 / 2$ and for all $q_{n}$ of degree at most $n$ the inequality

$$
\left\|g-q_{n}\right\|_{[0,1]} \leq C\left(\left\|g-q_{n}\right\|_{[a, 1]}+\omega_{[0,1]}^{r}(g, 1 / n)\right),
$$

with a $C$ that depends on $a$ and $n$. By iteration we obtain from here (7.8) for all $0<a<1$. 
Lemma 7.3 is Proposition 9.1 in [14], but since it plays a vital role in some of our reasonings, we give a new proof for it.

Proof. For all $L r / 2 n^{2} \leq x \leq 1-L r / 2 n^{2}$ the $r$-th difference $\Delta_{L / n^{2}}^{r} g(x)$ is defined and

$$
\left|\Delta_{L / n^{2}}^{r} g(x)\right| \leq \omega_{[0,1]}^{r}(g, \sqrt{4 L} / n) \leq C \omega_{[0,1]}^{r}(g, 1 / n)
$$

(note that on the right we have the $\varphi$-modulus of smoothness) since $L / n^{2} \leq$ $\sqrt{4 L} \sqrt{x(1-x)} / n$. Thus, for $2 L r / n^{2} \leq x \leq 1-2 L r / n^{2}$ we have

$$
\begin{aligned}
\left|\Delta_{L / n^{2}}^{r} q_{n}(x)\right| & \leq\left|\Delta_{L / n^{2}}^{r}\left(q_{n}-g\right)(x)\right|+\left|\Delta_{L / n^{2}}^{r} g(x)\right| \\
& \leq 2^{r}\left\|g-q_{n}\right\|_{\left[L / n^{2}, 1\right]}+C \omega_{[0,1]}^{r}(g, 1 / n)=: M
\end{aligned}
$$

so the norm of the left hand side over $\left[2 L r / n^{2}, 1-2 L r / n^{2}\right]$ is bounded by the right hand side, i.e. by $M$. But $\Delta_{L / n^{2}}^{r} q_{n}(x)$ is a polynomial of degree at most $n$, so it follows from Remez' inequality [11] ([1]) that then

$$
\left\|\Delta_{L / n^{2}}^{r} q_{n}\right\|_{[0,1]} \leq C_{1} M
$$

is also true with some $C_{1} \geq 1$ that depends only on $L$, and so for $\operatorname{Lr} / 2 n^{2} \leq x \leq$ $1 / 2$ we obtain

$$
\left|\Delta_{L / n^{2}}^{r}\left(g-q_{n}\right)(x)\right| \leq 2 C_{1} M
$$

But

$$
\begin{aligned}
& \Delta_{L / n^{2}}^{r}\left(g-q_{n}\right)(x)=(-1)^{r}\left(g\left(x-L r / 2 n^{2}\right)-q_{n}\left(x-L r / 2 n^{2}\right)\right) \\
& \quad+\sum_{j=0}^{r-1}(-1)^{j}\left(\begin{array}{l}
r \\
j
\end{array}\right)\left(g\left(x-L(r / 2-j) / n^{2}\right)-q_{n}\left(x-L(r / 2-j) / n^{2}\right)\right),
\end{aligned}
$$

and since all arguments $x-L(r / 2-j) / n^{2}, j=0,1, \ldots, r-1$, in the sum belong to $\left[L / n^{2}, 1\right]$, we can conclude (see also the definition of $M$ in (7.9))

$$
\left|g\left(x-\operatorname{Lr} / 2 n^{2}\right)-q_{n}\left(x-\operatorname{Lr} / 2 n^{2}\right)\right| \leq 2 C_{1} M+M,
$$

which completes the proof, since $x-L r / 2 n^{2}$ can be any point in $\left[0, L / n^{2}\right]$ by appropriately selecting $x \in\left[L r / 2 n^{2}, 1 / 2\right]$.

Thus, Theorem 6.5 follows if we can prove (7.2). Our approach will be to map $G_{P}$ into a cube, approximate on that cube (this was done already in 1987 in [6]) by some polynomials $Q_{n}\left(x_{1}, \ldots, x_{d-1}, u\right)$, and then go back to $G_{P}$ via the mapping

$$
\left(x_{1}, \ldots, x_{d-1}, u\right) \rightarrow\left(x_{1}, \ldots, x_{d-1}, \Phi\left(x_{1}, \ldots, x_{d}\right)\right) .
$$


Since $\Phi$ is a polynomial, this latter mapping will result in a polynomial approximant on the original $G_{P}$.

Before turning to details in connection with (7.2), we would like to make one more remark which simplifies things later. First of all, without loss of generality we may assume that the cube $I_{P}$ in the construction of $\Gamma_{P}$ is $[0,1]^{d-1}$ (this can always be achieved by rescaling). We have defined $G_{P}$ by attaching an $\underline{e}_{d}$ directional segment of fixed length $r_{0}$ to any point of $\Gamma_{P}$, but it is clear that the only thing we need of $G_{P}$ is that it covers a neighborhood (relative to $G$ ) of $\Gamma_{P}^{*}$. So instead of fixed length segments we can define $G_{P}$ by attaching an $\underline{e}_{d}$ directional segment to any $Q \in \Gamma_{P}$ of some positive length $l_{Q} \leq r_{0}$, where $l_{Q}$ is a continuous function of $Q$. This modified $G_{P}$ can serve the same purpose as the originally defined $G_{P}$, in particular, it will be enough to prove (7.2) for any such domain $G_{P}$.

It will be convenient to select this $G_{P}$ as follows. Let $\Phi$ be the polynomial that describes the boundary $\Gamma$ around $P \in \Gamma$ via $\Phi\left(x_{1}, \ldots, x_{d}\right)=0$. We have assumed that in a neighborhood of $P$, the domain $G$ lies "above" $\Gamma$ in the sense that if $\left(x_{1}, \ldots, x_{d-1}, x_{d}\right) \in \Gamma_{P}$, then for small $\varepsilon>0$ the point $\left(x_{1}, \ldots, x_{d-1}, x_{d}+\right.$ $\varepsilon$ ) belongs to $G$ (and then $\left(x_{1}, \ldots, x_{d-1}, x_{d}-\varepsilon\right)$ does not belong to $G$ ). We have also seen that $\partial \Phi / \partial x_{d} \neq 0$ on $\Gamma_{P}$, and we may assume that $\partial \Phi / \partial x_{d}>0$ (otherwise replace $\Phi$ by its negative). Then in a neighborhood of $P$ the domain $G$ is given by the inequality $\Phi \geq 0$, so for small $\delta>0$ all the points $\left(x_{1}, \ldots, x_{d}\right)$ with $0 \leq x_{1}, \ldots, x_{d-1} \leq 1$ and $0 \leq \Phi\left(x_{1}, \ldots, x_{d}\right) \leq \delta$ belong to $G$, and we choose $G_{P}$ as the set of all these points:

$$
G_{P}=\left\{\left(x_{1}, \ldots, x_{d}\right) \mid\left(x_{1}, \ldots, x_{d-1}\right) \in[0,1]^{d-1}, 0 \leq \Phi\left(x_{1}, \ldots, x_{d}\right) \leq \delta\right\} .
$$

This choice works just as well as the original one in (7.1), and actually in it $\delta>0$ can be as small as we wish.

\section{The $r=1$ case}

In this section we prove (7.2) in the special case $r=1$. Although later we shall give the proof for higher order moduli of smoothness, we feel it is instructive to separately give the proof for this simplest case because in that proof we may concentrate on the geometry involved in the problem, and the $r=1$ case holds all the necessary geometric reasoning without the technical details needed for higher order moduli. Later we will not repeat this geometric part in the more technical proof involving higher order smoothness.

So let $G_{0}$ be the part of $G$ which contains the points $\left(x_{1}, \ldots, x_{d}\right)$ with $\left(x_{1}, \ldots, x_{d-1}\right) \in[0,1]^{d-1}$ and $0 \leq \Phi\left(x_{1}, \ldots, x_{d}\right) \leq \delta$ (with the polynomial $\Phi$ that defines the boundary $\Gamma$ ) for some $\delta$. By replacing $\Phi$ by $\Phi / \delta$ we may assume $\delta=1$. As we have seen, in this domain $\partial \Phi / \partial x_{d}>0$, and we have the natural mapping $X=\left(x_{1}, \ldots, x_{d}\right) \rightarrow Y=\left(x_{1}, \ldots, x_{d-1}, u\right)$ from $G_{0}$ onto $[0,1]^{d}$ where $u=\Phi\left(x_{1}, \ldots, x_{d}\right)$. We can go from $Y$ to $X$ by setting $x_{d}=\chi_{x_{1}, \ldots, x_{d-1}}(u)$ for which

$$
\Phi\left(x_{1}, \ldots, x_{d-1}, \chi_{x_{1}, \ldots, x_{d-1}}(u)\right)=u .
$$


Clearly (use the inverse function theorem), this $\chi$ is real analytic in $x_{1}, \ldots, x_{d-1}, u$ with strictly positive derivative for $u \in[0,1]$. In fact, the assumed properties of $\Phi$ and the inverse function theorem imply positive upper and lower bounds for the derivative $\partial \chi_{x_{1}, \ldots, x_{d-1}}(u) / \partial u$ of $\chi_{x_{1}, \ldots, x_{d-1}}$ that are independent of $x_{1}, \ldots, x_{d-1}$. Another way to see that is to differentiate the equation (8.1) with respect to $u$.

If for fixed $\zeta_{1}, \ldots, \zeta_{d-1}$ we denote $\ell_{\zeta_{1}, \ldots, \zeta_{d-1}}$ the line $\left\{x_{1}=\zeta_{1}, \ldots, x_{d-1}=\right.$ $\left.\zeta_{d-1}\right\}$ in the $x_{d}$-direction, then the mapping $Y \rightarrow X$ maps the segment

$$
J_{\zeta_{1}, \ldots, \zeta_{d-1}}=[0,1]^{d} \cap \ell_{\zeta_{1}, \ldots, \zeta_{d-1}}
$$

onto the segment

$$
I_{\zeta_{1}, \ldots, \zeta_{d-1}}=G_{0} \cap \ell_{\zeta_{1}, \ldots, \zeta_{d-1}} .
$$

We also set $F(Y)=f(X)$, so $F$ is defined on $[0,1]^{d}$. On $J_{\zeta_{1}, \ldots, \zeta_{d-1}}$ we have

$$
F\left(\zeta_{1}, \ldots, \zeta_{d-1}, u\right)=f\left(\zeta_{1}, \ldots, \zeta_{d-1}, \chi_{\zeta_{1}, \ldots, \zeta_{d-1}}(u)\right),
$$

i.e.

$$
F\left(x_{1}, \ldots, x_{d-1}, \Phi\left(x_{1}, \ldots, x_{d}\right)\right)=f\left(x_{1}, \ldots, x_{d}\right),
$$

so we obtain from the from the aforementioned properties of $\chi_{\zeta_{1}, \ldots, \zeta_{d-1}}$ that

$$
\omega_{J_{\zeta_{1}, \ldots, \zeta_{d-1}}}^{1}(F, \delta) \sim \omega_{I_{\zeta_{1}, \ldots, \zeta_{d-1}}^{1}}^{1}(f, \delta)
$$

uniformly in $\left(\zeta_{1}, \ldots, \zeta_{d-1}\right) \in[0,1]^{d-1}$, i.e., the $u$-directional modulus of continuity of $F$ is bounded by the $x_{d}$-directional modulus of continuity of $f$. We emphasize that this is no longer true for higher order moduli of smoothness, for a nonlinear substitution does not preserve the size of those moduli (for example, the second order modulus of smoothness of $g(t)=t$ is identically 0 , but if we make any nonlinear substitution into $g$ then the second order modulus will not be 0$)$.

Consider now another coordinate direction, say the $x_{1}$-directional modulus of continuity of $F$. Note that the mapping $Y \rightarrow X$ does not change the 2 nd, $3 \mathrm{rd}, \ldots(d-1)$ st coordinates of a point. For fixed $\zeta_{2}, \ldots, \zeta_{d-1}, \zeta \in[0,1]$ the image of the segment $\left\{\left(x, \zeta_{2}, \ldots, \zeta_{d-1}, \zeta\right) \mid x \in[0,1]\right\}$ under the mapping $Y \rightarrow X$ is the intersection of $G_{0}$ with the polynomial curve

$$
\gamma_{\zeta_{2}, \ldots, \zeta_{d-1}, \zeta}:=\left\{\left(x_{1}, x_{d}\right) \mid \Phi\left(x_{1}, \zeta_{2}, \ldots, \zeta_{d-1}, x_{d}\right)=\zeta\right\}
$$

in the plane $\left\{x_{2}=\zeta_{2}, \ldots, x_{d-1}=\zeta_{d-1}\right\}$, and the mapping in between them is given by $x \rightarrow\left(x, \zeta_{1}, \ldots, \zeta_{d-1}, \chi_{x, \zeta_{1}, \ldots, \zeta_{d-1}}(\zeta)\right)$. There is a $c_{0}>0$ such that the distance from $\gamma_{\zeta_{2}, \ldots, \zeta_{d-1}, \zeta} \cap G_{0}$ to the boundary $\Gamma$ of $G$ is $\geq c_{0} \zeta$ (because $\left.\partial \Phi / \partial x_{d}>0\right)$. Since $Y \rightarrow X$ is a Lipshitz map, it follows that if $Y_{1}, Y_{2}$ are two points of distance $\leq 1 / n$ apart on the segment $\left\{\left(x, \zeta_{2}, \ldots, \zeta_{d-1}, \zeta\right) \mid x \in[0,1]\right\}$ and $X_{1}, X_{2}$ correspond to them under the mapping $Y \rightarrow X$, then $X_{1}, X_{2}$ are of distance $\leq D / n$, and if $\zeta \geq L / n^{2}$, then their distance from the boundary of $G$ is $\geq c_{0} L / n^{2}$. Let $r_{0}$ be the radius from the beginning of Section 6 and $C_{r_{0}}$ the circle 


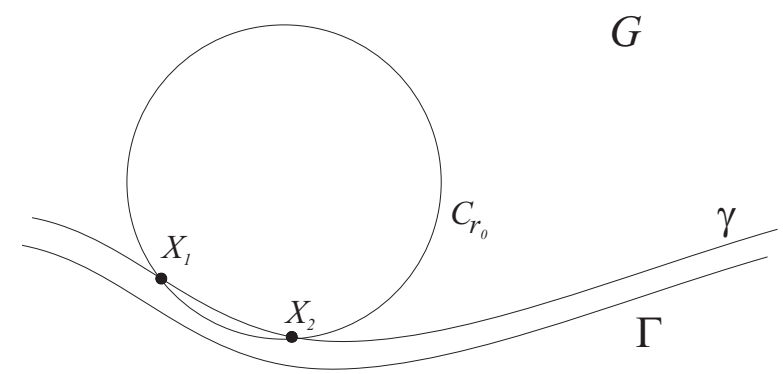

Figure 8: The points $X_{1}, X_{2}$ on the curve $\gamma=\gamma_{\zeta_{2}, \ldots, \zeta_{d-1}, \zeta}$ and the circle $C_{r_{0}}$ through them

in the plane $\left\{x_{2}=\zeta_{2}, \ldots, x_{d-1}=\zeta_{d-1}\right\}$ of radius $r_{0}$ which passes through $X_{1}$ and $X_{2}$ and which has its center above the curve $\gamma_{\zeta_{2}, \ldots, \zeta_{d-1}, \zeta}$ in that plane. We claim that for sufficiently large $L$ the circle $C_{r_{0}}$ lies inside $G$. Indeed, if $\sigma_{X_{1}, X_{2}}$ is its shorter arc in between $X_{1}$ and $X_{2}$, then $\sigma_{X_{1}, X_{2}}$ is of distance $\leq D_{1} / n^{2}$ - with some constant $D_{1}$ independent of $n$ and $\zeta_{2}, \ldots, \zeta_{d-1}, \zeta$ - from the arc of $\gamma_{\zeta_{2}, \ldots, \zeta_{d-1}, \zeta}$ lying in between $X_{1}$ and $X_{2}$. On the other hand, this latter arc lies of distance $\geq c_{0} L / n^{2}$ from the boundary, so for large $L$ the arc $\sigma_{X_{1}, X_{2}}$ lies inside $G$, see Figure 8. Furthermore, for large $n$ the other (longer) arc of $C_{r_{0}}$ in between $X_{1}$ and $X_{2}$ lies inside $G$ by the definition of $r_{0}$ from Section 6 , and these prove that $C_{r_{0}}$ lies, indeed, inside $G$.

Thus, by the definition of $\omega^{1}(f, \delta)_{G}$, we have

$$
\left|F\left(Y_{1}\right)-F\left(Y_{2}\right)\right|=\left|f\left(X_{1}\right)-f\left(X_{2}\right)\right| \leq \omega_{C_{r_{0}}}^{1}\left(f, D_{2} / n\right) \prec \omega^{1}(f, 1 / n)_{G} .
$$

This can be done with the other, $x_{2}, \ldots, x_{d-1}$ directions, as well, and it follows that $\omega_{[0,1]^{d-1} \times\left[L / n^{2}, 1\right]}^{1}(F, 1 / n)_{\left\{\underline{e}_{j}\right\}_{j=1}^{d}}$, the directional modulus of continuity of $F$ on $[0,1]^{d-1} \times\left[L / n^{2}, 1\right]$ in the coordinate directions, is bounded by an absolute constant times $\omega^{1}(f, 1 / n)_{G}$. Now we can invoke $[6$, Theorem 12.1.1] for the rectangular parallelepiped $[0,1]^{d-1} \times\left[L / n^{2}, 1\right]$, according to which there are polynomials $Q_{n}\left(x_{1}, \ldots, x_{d-1}, u\right)$ of degree at most $n$ such that

$$
\left\|F-Q_{n}\right\|_{[0,1]^{d-1} \times\left[L / n^{2}, 1\right]} \leq C \omega_{[0,1]^{d-1} \times\left[L / n^{2}, 1\right]}^{1}(F, 1 / n)_{\left\{\underline{e}_{j}\right\}_{j=1}^{d}} \leq C \omega^{1}(f, 1 / n)_{G} .
$$

On substituting here $u=\Phi\left(x_{1}, \ldots, x_{d}\right)$ we obtain

$$
\left|f\left(x_{1}, \ldots, x_{d}\right)-Q_{n}\left(x_{1}, \ldots, x_{d-1}, \Phi\left(x_{1}, \ldots, x_{d}\right)\right)\right| \leq C \omega^{1}(f, 1 / n)_{G}
$$

on the part of $G_{0}$ which satisfies $\Phi\left(x_{1}, \ldots, x_{d}\right) \geq L / n^{2}$. Here the function $Q_{n}\left(x_{1}, \ldots, x_{d-1}, \Phi\left(x_{1}, \ldots, x_{d}\right)\right)$ is a polynomial of degree $\leq n \cdot \operatorname{deg}(\Phi)$. On every $x_{d}$-directional segment $I=I_{\zeta_{1}, \ldots, \zeta_{d-1}} \subset G_{0}$ we have $u=\Phi\left(x_{1}, \ldots, x_{d}\right) \geq L / n^{2}$ for all points but those lying on a segment of the "lower" part of $I$ (in the $x_{d}$ direction) of length $\leq C_{0} L / n^{2}$ (with some $C_{0}$ independent of $L$ and $n$ ), so (8.2) is true for all such points, and from that we can conclude from Lemma 7.3 that 
(8.2) pertains to hold on the whole of $G_{0}$. This proves (7.2) in the special case when $r=1$.

\section{Approximation on disks}

In this section we consider the special case when $G$ is the disk

$$
G:=\left\{\left(x_{1}, x_{2}\right) \mid R_{0}^{2}-\left(x_{1}-1 / 2\right)^{2}-x_{2}^{2} \geq 0\right\},
$$

i.e., $d=2$ and $\Phi\left(x_{1}, x_{2}\right)=R_{0}^{2}-\left(x_{1}-1 / 2\right)^{2}-x_{2}^{2}$, and prove Theorem 6.5 , more precisely $(7.2)$, in this case.

This section is devoted solely to disks. This special domain will be used in an essential way when proving the general case. Furthermore, several estimates on the moduli of smoothness of transformed functions that we do in this section work also for general domains and they will not be repeated (just mentioned with reference to this section) in final part of the proof.

A typical boundary domain from Section 7 for this $G$ is

$$
\left\{\left(x_{1}, x_{2}\right) \mid a \leq x_{1} \leq b, R_{0}^{2}-\delta \leq\left(x_{1}-1 / 2\right)^{2}+x_{2}^{2} \leq R_{0}^{2}, x_{2} \leq 0\right\}
$$

with some $1 / 2-R_{0}<a<b<1 / 2+R_{0}$ and small $\delta>0$, and it is sufficient to show the possibility of Jackson type approximation on such a domain. For simplicity we set $[a, b]=[0,1]$ and $\delta=1$ (the reasoning in the general case would be the same), and again for convenience we assume $R_{0} \geq 1$. So let

$$
G_{0}:=\left\{\left(x_{1}, x_{2}\right) \mid 0 \leq x_{1} \leq 1, R_{0}^{2}-1 \leq\left(x_{1}-1 / 2\right)^{2}+x_{2}^{2} \leq R_{0}^{2}, x_{2} \leq 0\right\},
$$

see Figure 9.

Under the mapping $X \rightarrow Y,\left(x_{1}, x_{2}\right) \rightarrow\left(x_{1}, u\right), u=R_{0}^{2}-\left(x_{1}-1 / 2\right)^{2}-x_{2}^{2}$, discussed in Section 8 this $G_{0}$ is mapped into $[0,1]^{2}$, and if we set $F(Y)=f(X)$, then we are going to show that

$$
\omega_{[0,1]^{2}}^{r}(F, 1 / n)_{\left\{\underline{e}_{j}\right\}_{j=1}^{2}} \prec \omega^{r}(f, 1 / n)_{G} .
$$

Recall that on the left the modulus is the directional modulus of smoothness (defined in (1.6) on $[0,1]^{2}$, see also (1.7) and (1.1)) using the $x_{1}$ and $x_{2}(\equiv u$ ) directions on $[0,1]^{2}$, while on the right $\omega^{r}(f, 1 / n)_{G}$ is the modulus of smoothness defined in Section 6. First we discuss the directional modulus $\omega^{r}(F, 1 / n)_{\underline{e}_{1}}$ of $F$ in the $x_{1}$ direction.

For simplicity, in what follows we write $\omega(1 / n)$ for $\omega^{r}(f, 1 / n)_{G}$.

Let $C_{R}$ be the circle about $(1 / 2,0)$ and of radius $R \leq R_{0}$ with $R^{2} \geq R_{0}^{2}-1$. By the definition of the modulus of smoothness, the $2 \pi$-periodic function

$$
g(\theta)=f(1 / 2+R \sin \theta,-R \cos \theta)
$$

has $r$-th modulus of smoothness $\leq \omega^{r}(f, \delta)_{G}$, hence it can be approximated by trigonometric polynomials $T_{n}$ of degree at most $n$ in the order $\omega(1 / n): \| g-$ 


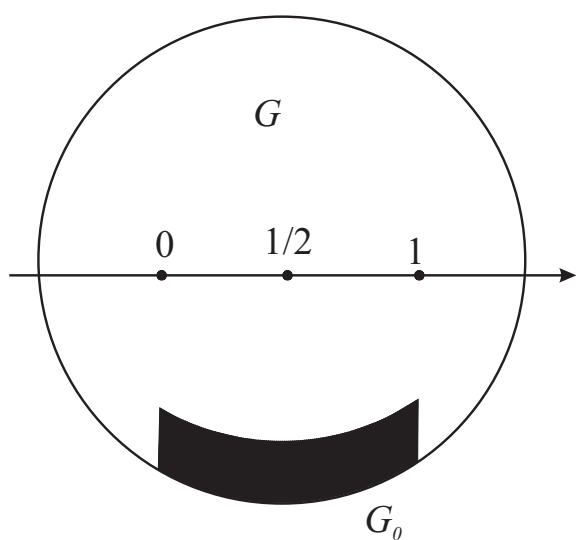

Figure 9:

$T_{n} \| \prec \omega(1 / n)$ (see [5, Theorem 7.2.3]). Then we also have $\omega^{r}\left(T_{n}, 1 / n\right) \prec \omega(1 / n)$ (here $\omega^{r}$ is the classical modulus of smoothness of the trigonometric polynomial $\left.T_{n}\right)$, and since, by Stechkin's theorem [13], $\left\|T_{n}^{(r)}\right\| \prec n^{r} \omega^{r}\left(T_{n}, 1 / n\right)$, it follows that $\left\|T_{n}^{(r)}\right\| \prec n^{r} \omega^{r}(1 / n)$.

If $r-1 \geq 1$, then $T_{n}^{(r-1)}$, being a trigonometric polynomial without a constant term, vanishes somewhere, say $T_{n}^{(r-1)}\left(\theta_{0}\right)=0, \theta_{0} \in[0,2 \pi]$. But then for all other $\theta \in[0,2 \pi]$ we have

$$
\left|T_{n}^{(r-1)}(\theta)\right|=\left|\int_{\theta_{0}}^{\theta} T_{n}^{(r)}(u) d u\right| \leq 2 \pi\left\|T_{n}^{(r)}\right\| \prec n^{r} \omega(1 / n) .
$$

Repeating this argument using $T_{n}^{(r-1)}$ in place of $T_{n}^{(r)}$ we get $\left\|T_{n}^{(r-2)}\right\| \prec$ $n^{r} \omega(1 / n)$, and so on, we can infer for all $1 \leq j \leq r$

$$
\left\|T_{n}^{(j)}\right\| \prec n^{r} \omega(1 / n) .
$$

Now consider $F\left(x_{1}, u\right)$ on the horizontal segment $u=R_{0}^{2}-R^{2}$ of $[0,1] \times[0,1]$. It has the form

$$
F\left(x_{1}, R_{0}^{2}-R^{2}\right)=f\left(x_{1},-\sqrt{R^{2}-\left(x_{1}-1 / 2\right)^{2}}\right)=g\left(\arcsin \left(\left(x_{1}-1 / 2\right) / R\right)\right) .
$$

Thus,

$$
\left|\Delta_{h}^{r} F\left(x_{1}, R_{0}^{2}-R^{2}\right)\right| \leq\left|\Delta_{h}^{r} T_{n}\left(\arcsin \left(\left(x_{1}-1 / 2\right) / R\right)\right)\right|+2^{r}\left\|g-T_{n}\right\|,
$$

which can be estimated as (see e.g. [5, Chapter 2, (7.10)])

$$
|h|^{r}\left\|\left(T_{n}\left(\arcsin \left(\left(x_{1}-1 / 2\right) / R\right)\right)\right)^{(r)}\right\|_{[0,1]}+O(\omega(1 / n)) .
$$


Since, by Faà di Bruno's formula (cf. [10, Theorem 1.3.2]) the norm on the right is at most $C \sum_{j=1}^{r}\left\|T_{n}^{(j)}\right\|$, for $h \leq 1 / n$ we obtain from (9.2) that

$$
\left|\Delta_{h}^{r} F\left(x_{1}, R_{0}^{2}-R^{2}\right)\right| \prec \omega(1 / n),
$$

which is stronger than

$$
\omega_{[0,1]^{2}}^{r}(F, 1 / n)_{\underline{e}_{1}} \prec \omega(1 / n)
$$

(recall that the modulus of smoothness on the left of (9.3) was defined in (1.6) and it involves a quantity smaller than 1 in the step-size $\cdot$ of $\left.\Delta^{r} F\right)$.

Before going on, let us record here the following consequence:

Corollary 9.1 Let $G$ be any domain in $\mathbf{R}^{d}$ as discussed in Section 6, and let $C_{R}$ be any circle that lies in $G$ together with its interior which is parallel with a coordinate plane. If $P_{1}$ and $P_{2}$ are any two points of $C_{R}$, then for any $n=1,2, \ldots$,

$$
\left|f\left(P_{1}\right)-f\left(P_{2}\right)\right| \leq C_{R} n^{r} \omega^{r}(f, 1 / n)_{G},
$$

and here the constant $C_{R}$ depends only on $G$ and the radius of the circle in question.

Proof. We have essentially proved this above: choose a $T_{n}$ as before with $\left\|f-T_{n}\right\| \prec \omega^{r}(f, 1 / n)_{G}$ on $C_{R}$, and use that

$$
\left|T_{n}\left(P_{1}\right)-T_{n}\left(P_{2}\right)\right| \leq C_{R} n^{r} \omega^{r}(f, 1 / n)_{G}
$$

is a consequence of $\left\|T_{n}^{\prime}\right\| \prec n^{r} \omega^{r}(f, 1 / n)_{G}$ (see (9.2)).

Next, we consider the modulus of smoothness of $F$ in the $\underline{e}_{2}$ (i.e., $u=x_{2}$ ) direction. For a $\zeta_{1}$ let $\ell_{\zeta_{1}}$ be the $x_{1}=\zeta_{1}$ line, and, as in Section 8 , let $J=J_{\zeta_{1}}=$ $\ell_{\zeta_{1}} \cap[0,1]^{2}$ and $I=I_{\zeta_{1}}=\ell_{\zeta_{1}} \cap G_{0}$, and in what follows we keep the notations established there. We have seen that the mapping $Y \rightarrow X$, when restricted to $J$, is given by a real analytic function $\chi(u)=\chi_{\zeta_{1}}(u)$ with positive derivative in $u$ (which is uniformly bounded from below and above in $\zeta_{1}$ ).

By definition, $\omega_{I}^{r}(f, 1 / n) \leq \omega^{r}(f, 1 / n)_{G}$, and by the equivalence of the $\varphi$ modulus of smoothness with the $K$-functional (see Theorem [6, Theorem 2.1.1]) there is a $g$ defined on $I$ such that

$$
\omega_{I}^{r}(f, 1 / n) \prec\|f-g\|_{I}+\frac{1}{n^{r}}\left\|\varphi_{I}^{r} g^{(r)}\right\|_{I} \prec \omega_{I}^{r}(f, 1 / n) \prec \omega(1 / n),
$$

where $\varphi_{I}$ is the $\varphi$ function for $I$ (i.e., if the endpoints of $I$ are $\left(\zeta_{1}, a\right)$ and $\left(\zeta_{1}, b\right)$, then $\left.\varphi_{I}\left(\zeta_{1}, u\right)=\sqrt{|u-a||u-b|}\right)$ and the differentiation in $g^{(r)}$ is understood in the $x_{2}$-direction, so $g^{(r)} \equiv \partial^{r} g / \partial x_{2}^{r}$. Furthermore, the $\prec$ in (9.4) is uniform in $I$. This $g$ will be used only on $I$, so we shall think of it as a function of a single variable. 
Let $\tau_{1}, \ldots, \tau_{r}$ be equidistant points in the middle third of $I$ of consecutive distance $|I| / 3 r$. Any two consecutive points $\tau_{j}, \tau_{j+1}$, lie on a circle of radius $|I| / 6 r$ that lies in $G$, so, by Corollary 9.1, we have $\left|f\left(\tau_{j+1}\right)-f\left(\tau_{j}\right)\right| \prec n^{r} \omega(1 / n)$. Then, in view of (9.4), the same is true for $g:\left|g\left(\tau_{j+1}\right)-g\left(\tau_{j}\right)\right| \prec n^{r} \omega(1 / n)$. But then the mean value theorem gives that $g^{\prime}=\partial g / \partial x_{2}$ is $\prec n^{r} \omega(1 / n)$ somewhere in between $\tau_{1}$ and $\tau_{2}$. Using higher order mean value theorems (i.e. that $\Delta_{h}^{j} w=$ $h^{r} w^{(j)}(\xi)$ for some $\xi$ ) we can conclude the same way that for all $1 \leq j \leq r$ there is a $\xi_{j}$ in the middle third of $I$ such that

$$
g^{(j)}\left(\xi_{j}\right) \prec n^{r} \omega(1 / n) .
$$

Now if $\xi \in I$ lies below $v \in I$ (in the $x_{2}$ direction) and at the same time $v$ lies below $\xi_{r-1}$, then $\varphi_{I}(\xi) \leq C \varphi_{I}(v)$ with an absolute constant (actually, $C=3 / 2 \sqrt{2}$ works). Here we used that $\xi_{r-1}$ belongs to the middle third of $I$. Therefore, we obtain for $\xi$ lying below $\xi_{r-1}$

$$
\begin{aligned}
\varphi_{I}(\xi)^{r}\left|g^{(r-1)}(\xi)\right| & \leq \varphi_{I}(\xi)^{r}\left|\int_{\xi}^{\xi_{r-1}} g^{(r)}(v) d v\right|+\varphi_{I}(\xi)^{r}\left|g^{(r-1)}\left(\xi_{r-1}\right)\right| \\
& \prec\left|\int_{\xi}^{\xi_{r-1}} \varphi_{I}(v)^{r}\right| g^{(r)}(v)|d v|+n^{r} \omega(1 / n) \prec n^{r} \omega(1 / n),
\end{aligned}
$$

where we used (9.4), and the choice of $\xi_{r-1}$. A similar estimate is true if $\xi$ lies above $\xi_{r-1}$. Since the same reasoning can be given for $g^{(r-2)}$ (using $g^{(r-2)}\left(\xi_{r-2}\right) \prec n^{r} \omega(1 / n)$ and the just proven relation), we obtain $\varphi_{I}^{r}\left|g^{(r-2)}\right| \prec$ $n^{r} \omega(1 / n)$, and in general, for all $j=1,2, \ldots, r$ we have

$$
\varphi_{I}^{r}\left|g^{(j)}\right| \prec n^{r} \omega(1 / n) .
$$

On the segment $J$ the function $F$ is given by $\left.f\left(\zeta_{1}, \chi(u)\right)\right)$, so it is natural to consider $G(u)=g(\chi(u))$. For it we have

$$
\|F-G\|_{J}=\|f-g\|_{I} .
$$

Since $\varphi_{J}(u) \sim \varphi_{I}(\chi(u))$, it follows from the Faà di Bruno formula ([10, Theorem 1.3.2]) that

$$
\left\|\varphi_{J}^{r} \frac{\partial^{r} G}{\partial u^{r}}\right\|_{J} \prec \sum_{j=1}^{r}\left\|\varphi_{I}(\chi)^{r} g^{(j)}(\chi)\right\|_{J} \prec n^{r} \omega(1 / n),
$$

where the last relation follows from (9.5). Thus, using the equivalence relation between the $K$-functional and the $\varphi$-moduli of smoothness (see [6, Theorem 2.1.1]) we obtain from (9.4), (9.6) and (9.7)

$$
\omega_{J}^{r}(F, 1 / n)_{\underline{e}_{2}} \prec\|F-G\|_{J}+\frac{1}{n^{r}}\left\|\varphi_{J}^{r} \frac{\partial^{r} G}{\partial u^{r}}\right\|_{J} \prec \omega(1 / n) .
$$

Here $J=J_{\zeta_{1}}$ with $\zeta_{1} \in[0,1]$ arbitrary, so we can conclude

$$
\omega_{[0,1]^{2}}^{r}(F, 1 / n)_{\underline{e}_{2}} \prec \omega(1 / n) .
$$


Remark 9.2 Before going on with the discussion of approximation on the disk let us remark that the preceding proof for estimating $\omega_{\left[0,11^{2}\right.}^{r}(F, 1 / n)_{e_{2}}$ did not use that the boundary was a disk, it works for any polynomial curve as in Section 8 (just note that the small circles through the points $\tau_{j}$ are available also in that case, as well). What is more, the dimension need not be 2 , either. Indeed, if the dimension is $d$, then the segments $I_{\zeta_{1}, \ldots, \zeta_{d-1}}$ and $J_{\zeta_{1}, \ldots, \zeta_{d-1}}$ (see Section 8) lie in the plane $\left\{x_{2}=\zeta_{2}, \ldots, x_{d-1}=\zeta_{d-1}\right\}$, and the preceding proof can be carried out in that plane using circles and directional moduli belonging to that plane. So we have (9.8) in general:

$$
\omega_{[0,1]^{d}}^{r}(F, 1 / n)_{\underline{e}_{d}} \prec \omega^{r}(f, 1 / n)_{G} .
$$

Now we return to the proof of (7.2) for the disk that we are considering in this section. From (9.3) and (9.8) we can conclude, using Theorem [6, Theorem 12.1.1] (or Theorem 1.1 from the first part of this paper) for the square $[0,1]^{2}$, that there are polynomials $Q_{n}\left(x_{1}, u\right)$ of degree at most $n$ such that for all $(x, u) \in$ $[0,1]^{2}$

$$
\left|F(x, u)-Q_{n}(x, u)\right| \leq C\left(\omega_{[0,1]^{2}}^{r}(F, 1 / n)_{\underline{e}_{1}}+\omega_{[0,1]^{2}}^{r}(F, 1 / n)_{\underline{e}_{2}}\right) \prec \omega(1 / n) .
$$

On substituting here $u=\Phi\left(x_{1}, x_{2}\right)=R_{0}^{2}-\left(x_{1}-1 / 2\right)^{2}-x_{2}^{2}$, we obtain

$$
\left|f\left(x_{1}, x_{2}\right)-Q_{n}\left(x_{1}, R_{0}^{2}-\left(x_{1}-1 / 2\right)^{2}-x_{2}^{2}\right)\right| \prec \omega^{r}(f, 1 / n)_{G}
$$

on $G_{0}$. According to Section 7, this is enough to conclude $E_{n}(f)_{G} \prec \omega^{r}(f, 1 / n)_{G}$.

\section{A detour on the derivative of composite func- tions}

Let $P(x, y)=P_{n}(x, y)$ be a polynomial of two variables and $\sigma(t)$ a one-variable infinitely many times differentiable function. For $j=0,1, \ldots$ we set

$$
D_{j}(P, t)=D_{j}(P, \sigma, t)=\sum_{s=0}^{j}\left(\begin{array}{l}
j \\
s
\end{array}\right) \frac{\partial^{j} P(t, \sigma(t))}{\partial x^{j-s} \partial y^{s}}\left(\sigma^{\prime}(t)\right)^{s},
$$

where $D_{0}(P, t)=P(t)$. For these straightforward differentiation yields

$$
\frac{d D_{j}(P, t)}{d t}=D_{j+1}(P, t)+j D_{j-1}\left(\frac{\partial P}{\partial y}, t\right) \sigma^{\prime \prime}(t)
$$

and hence the derivative with respect to $t$ of an expression of the form

$$
D_{j}\left(\frac{\partial^{l} P}{\partial y^{l}}, t\right)\left(\sigma^{\prime \prime}(t)\right)^{k_{2}}\left(\sigma^{\prime \prime \prime}(t)\right)^{k_{3}} \cdots\left(\sigma^{(r)}(t)\right)^{k_{r}}
$$


is the sum of the terms

$$
\begin{gathered}
D_{j+1}\left(\frac{\partial^{l} P}{\partial y^{l}}, t\right)\left(\sigma^{\prime \prime}(t)\right)^{k_{2}}\left(\sigma^{\prime \prime \prime}(t)\right)^{k_{3}} \cdots\left(\sigma^{(r)}(t)\right)^{k_{r}}, \\
j D_{j-1}\left(\frac{\partial^{l+1} P}{\partial y^{l+1}}, t\right)\left(\sigma^{\prime \prime}(t)\right)^{k_{2}+1}\left(\sigma^{\prime \prime \prime}(t)\right)^{k_{3}} \cdots\left(\sigma^{(r)}(t)\right)^{k_{r}}
\end{gathered}
$$

and

$$
\begin{aligned}
& D_{j}\left(\frac{\partial^{l} P}{\partial y^{l}}, t\right)\left(k_{2}\left(\sigma^{\prime \prime}(t)\right)^{k_{2}-1}\left(\sigma^{\prime \prime \prime}(t)\right)^{k_{3}+1} \quad \ldots \quad\left(\sigma^{(r)}(t)\right)^{k_{r}}\right. \\
& \left.+\cdots+\left(\sigma^{\prime \prime}(t)\right)^{k_{2}}\left(\sigma^{\prime \prime \prime}(t)\right)^{k_{3}} \quad \cdots \quad k_{r}\left(\sigma^{(r)}(t)\right)^{k_{r}-1} \sigma^{(r+1)}(t)\right) .
\end{aligned}
$$

Since

$$
\frac{d P(t, \sigma(t))}{d t}=D_{1}(P, t)
$$

is also true, it follows by induction on $m=1,2, \ldots$ that every derivative $d^{m} P(t, \sigma(t)) / d t^{m}$ is a linear combinations of terms of the form (10.2) with $j+2 l \leq m$. Furthermore, the only terms of the form (10.2) with $j+2 l=m$ that appear in $d^{m} P(t, \sigma(t)) / d t^{m}$ are the terms (see (10.1))

$$
D_{m}(P, t), \quad D_{m-2}\left(\frac{\partial P}{\partial y}, t\right) \sigma^{\prime \prime}(t), \quad D_{m-4}\left(\frac{\partial^{2} P}{\partial y^{2}}, t\right)\left(\sigma^{\prime \prime}(t)\right)^{2}, \cdots,
$$

and since there is no cancellation above in the differentiation (c.f. (10.1)), these terms do appear with some positive coefficients.

Suppose now that for some $r \geq 1$ we have, besides $\sigma$, infinitely many times differentiable functions $\sigma_{1}, \ldots, \sigma_{r}$ with the property that at some point $t_{0}$ we have $\sigma_{\tau}^{\prime}\left(t_{0}\right)=\sigma^{\prime}\left(t_{0}\right)$ for all $\tau=1, \ldots, r$, but the second derivatives of the $\sigma_{\tau}$ 's at $t=t_{0}$ are all different: if $\sigma_{\tau}^{\prime \prime}\left(t_{0}\right)=\rho_{\tau}$, then $\rho_{\tau} \neq \rho_{\nu}$ if $\tau \neq \nu$. Suppose also that for some polynomials $P_{n}(x, y), n=1,2, \ldots$ we have the bounds

$$
\left.\frac{d^{m} P_{n}\left(t, \sigma_{\tau}(t)\right)}{d t^{m}}\right|_{t=t_{0}} \prec \Omega_{n}
$$

for all $\tau=1, \ldots, r$ and $1 \leq m \leq r$ with some sequence $\left\{\Omega_{n}\right\}$. We claim that under these assumptions we also have

$$
\left.\frac{d^{r} P_{n}(t, \sigma(t))}{d t^{r}}\right|_{t=t_{0}} \prec \Omega_{n}
$$

and here the $\prec$ depends only the $\prec$ in (10.3) and on the numbers $\rho_{1}, \ldots, \rho_{r}$.

Indeed, since we have $\sigma_{\tau}^{\prime}\left(t_{0}\right)=\sigma^{\prime}\left(t_{0}\right)$, the expressions $D_{j}\left(\frac{\partial^{l} P_{n}}{\partial y^{2}}, \sigma_{\tau}, t_{0}\right), \tau=$ $0, \ldots, r$, are all the same if we set $\sigma_{0}=\sigma$. Therefore, in view of what has been said before, to prove (10.4) it is sufficient to show that for all $j, l$ with $j+2 l \leq r$ we have

$$
D_{j}\left(\frac{\partial^{l} P_{n}}{\partial y^{l}}, t_{0}\right) \prec \Omega_{n},
$$


which we are going to prove by induction on $j+2 l$. First of all, when $j+2 l=1$ then necessarily $j=1$ and $l=0$, and in this case (recall that $\sigma=\sigma_{0}$ )

$$
\begin{aligned}
D_{j}\left(\frac{\partial^{l} P_{n}}{\partial y^{l}}, t_{0}\right)=D_{1}\left(P_{n}, t_{0}\right) & =D_{1}\left(P_{n}, \sigma_{0}, t_{0}\right)=D_{1}\left(P_{n}, \sigma_{1}, t_{0}\right) \\
& =\left.\frac{d P_{n}\left(t, \sigma_{1}(t)\right)}{d t}\right|_{t=t_{0}} \prec \Omega_{n}
\end{aligned}
$$

by the assumption (10.3).

Suppose now that $(10.5)$ is true for all $j+2 l<m(\leq r)$. As we have seen,

$$
\begin{aligned}
\left.\frac{d^{m} P_{n}\left(t, \sigma_{\tau}(t)\right)}{d t^{m}}\right|_{t=t_{0}} & =D_{m}\left(P_{n}, t\right)+c_{m, 1} D_{m-2}\left(\frac{\partial P_{n}}{\partial y}, 0\right) \sigma_{\tau}^{\prime \prime}\left(t_{0}\right) \\
& +c_{m, 2} D_{m-4}\left(\frac{\partial^{2} P_{n}}{\partial y^{2}}, 0\right)\left(\sigma_{\tau}^{\prime \prime}\left(t_{0}\right)\right)^{2}+\cdots+\text { terms with } j+2 l<m
\end{aligned}
$$

where the coefficients $c_{m, s}$ are non-zero. By the induction hypothesis here all the terms with $j+2 l<m$ are $\prec \Omega_{n}$, and by assumption the left hand side is also $\prec \Omega_{n}$ for $\tau=1, \ldots, r$. Therefore, in view of the notation $\sigma_{\tau}^{\prime \prime}\left(t_{0}\right)=\rho_{\tau}$, we have

$D_{m}\left(P_{n}, t_{0}\right)+c_{m, 1} D_{m-2}\left(\frac{\partial P_{n}}{\partial y}, t_{0}\right) \rho_{\tau}+c_{m, 2} D_{m-4}\left(\frac{\partial^{2} P_{n}}{\partial y^{2}}, t_{0}\right) \rho_{\tau}^{2}+\cdots \prec \Omega_{n}, \quad \tau=1, \ldots, r$.

Using the first ${ }^{6}[m / 2]+1$ of these relations we obtain an $([m / 2]+1) \times([m / 2]+$ 1) size linear system of equations for $c_{m, s} D_{m-2 s}\left(\frac{\partial^{s} P_{n}}{\partial y^{s}}, 0\right), s=0, \ldots,[m / 2]$, with non-zero determinant (a Vandermonde determinant in $\rho_{1}, \rho_{2}, \ldots, \rho_{[m / 2]+1}$, which numbers are different by assumption) and with right hand side consisting of numbers $\prec \Omega_{n}$. Solving this system we obtain that all the terms $c_{m, s} D_{m-2 s}\left(\frac{\partial^{s} P_{n}}{\partial y^{s}}, t_{0}\right)$ are $\prec \Omega_{n}$. Since $c_{m, s} \neq 0$, this completes the induction step, and the proof of (10.4) is complete.

What we have just proven, we shall use in Section 11 in the following situation. Suppose that $D_{r_{0}}$ is a disk on the plane. Let $\sigma:=(t, \sigma(t))$ be a $\mathbf{C}^{\infty}$ curve that lies in $D_{r_{0}}$, and suppose that $D_{\rho} \subset D_{r_{0}}$ is a disk that touches (i.e. its boundary touches) $\sigma$ at some point $\left(t_{0}, \sigma\left(t_{0}\right)\right)$. For a given $r \geq 1$ fix $r$ different radii $\rho / 2 \leq R_{\tau} \leq \rho, 1 \leq \tau \leq r$, and let $C_{R_{\tau}}$ be the circle of radius $R_{\tau}$ that lies in $D_{\rho}$ and touches $\sigma$ at the point $\left(t_{0}, \sigma\left(t_{0}\right)\right)$. If $C_{R_{\tau}}$ is the circle $\left(x-x_{\tau}\right)^{2}+\left(y-y_{\tau}\right)^{2}=R_{\tau}^{2}$, then in a neighborhood of the point $\left(t_{0}, \sigma\left(t_{0}\right)\right)$ it has parametrization $\left(t, \sigma_{\tau}(t)\right)$ with

$$
\sigma_{\tau}(t)=y_{\tau}-\sqrt{R_{\tau}^{r}-\left(t-x_{\tau}\right)^{2}}
$$

(note that by the form of $\sigma$, the common tangent line to $\sigma$ and $D_{\rho}$ at $\left(t_{0}, \sigma\left(t_{0}\right)\right.$ ) is not vertical) with possibly + replacing - in front of the square root sign, but

\footnotetext{
$6[\cdot]$ denotes integral part
} 
in what follows we shall keep the - sign. Now

$$
\begin{gathered}
\sigma_{\tau}^{\prime}\left(t_{0}\right)=\frac{t-x_{\tau}}{\sqrt{R_{\tau}^{2}-\left(t-x_{\tau}\right)^{2}}}, \\
\sigma_{\tau}^{\prime \prime}\left(t_{0}\right)=\frac{R_{\tau}^{2}}{\left(R_{\tau}^{2}-\left(t-x_{\tau}\right)^{2}\right)^{3 / 2}}=\frac{1}{R_{\tau}}\left(1+\sigma_{\tau}^{\prime}\left(t_{0}\right)^{2}\right)^{3 / 2} .
\end{gathered}
$$

These $\sigma_{\tau}$ touch $\sigma$ at $\left(t_{0}, \sigma\left(t_{0}\right)\right)$, so they have the same derivative at $t_{0}$ as $\sigma$ : $\sigma^{\prime}\left(t_{0}\right)=\sigma_{\tau}^{\prime}\left(t_{0}\right)$ (this follows from the fact that the tangent direction to $\sigma_{\tau}$ at $\sigma_{\tau}\left(t_{0}\right)$ is $\left.\left(1, \sigma_{\tau}^{\prime}\left(t_{0}\right)\right)\right)$. But then (10.6) shows that the second derivatives are different at $t_{0}: \sigma_{\tau}^{\prime \prime}\left(t_{0}\right) \neq \sigma_{\tau}^{\prime \prime}\left(t_{0}\right)$ because the $R_{\tau}, \tau=1, \ldots, r$ are different.

Let now $f$ be a function on a domain $G$ that contains $D_{r_{0}}$, and let $P_{n}(x, y)$ be polynomials (of degree $n=1,2, \ldots$ ) of two variables such that $\left\|f-P_{n}\right\|_{D_{r_{0}}} \prec$ $\omega(1 / n)$, where $\omega(1 / n)$ is the $r$-th modulus of smoothness of $f$ on $G$. In Section 9 it was shown that for every $r \geq 1$ there are such polynomials $P_{n}$. The polynomial $P_{n}$, when restricted to either of the circles $C_{R_{\tau}}$, gives a trigonometric polynomial

$$
T_{n}(\theta)=P_{n}\left(x_{\tau}+R_{\tau} \sin \theta, y_{\tau}-R_{\tau} \cos \theta\right) .
$$

For this $T_{n}$ it was shown in Section 9 (see (9.2)) that

$$
\left\|T_{n}^{(j)}\right\| \prec n^{r} \omega(1 / n) \quad \text { for all } j=1,2, \ldots, r,
$$

and here $\prec$ depends only on $R_{\tau}$. The expression $P_{n}\left(t, \sigma_{\tau}(t)\right)$ is $T_{n}(\arcsin ((t-$ $\left.x_{\tau}\right) / R_{\tau}$ ), so from (10.7) and from Faà di Bruno's formula (cf. [10, Theorem 1.3.2]) we obtain

$$
\left.\frac{d^{m} P_{n}\left(t, \sigma_{\tau}(t)\right)}{d t^{m}}\right|_{t=t_{0}} \prec n^{r} \omega(1 / n)
$$

for all $1 \leq m \leq r$. Since this is true uniformly for all $\tau=1, \ldots, r$, we are in the situation of (10.3) with $\Omega_{n}=n^{r} \omega(1 / n)$, so from (10.4) we can conclude

$$
\left.\frac{d^{r} P_{n}(t, \sigma(t))}{d t^{r}}\right|_{t=t_{0}} \prec n^{r} \omega(1 / n) .
$$

\section{Proof of Theorem 6.5}

According to the general setup in Section 7, it is sufficient to prove (7.2), i.e. the possibility of Jackson-rate approximation on the sets $G_{P}$. So let $G_{0}$ be such a set, and without loss of generality we may assume that $G_{0}$ is positioned as in the discussion leading to $(7.2)$, i.e. it is the union of some $x_{d}$-directional segments over a small part of the boundary $G$. As in Section 8, the closed domain $G_{0}$ corresponds to $[0,1]^{d}$ under the mapping $X \rightarrow Y$, and $f$ corresponds to $F(Y)=f(X)$.

As in Sections 8-9 it is sufficient to show that the directional modulus of smoothness

$$
\omega_{[0,1]^{d}}^{r}(F, \delta)_{\left\{\underline{e}_{i}\right\}_{i=1}^{d}}=\max _{1 \leq i \leq d} \omega_{[0,1]^{d}}^{r}(F, \delta)_{\underline{e}_{i}}
$$


of $F$ satisfies

$$
\omega_{[0,1]^{d}}^{r}(F, 1 / n)_{\left\{\underline{e}_{i}\right\}_{i=1}^{d}} \leq C \omega^{r}(f, 1 / n)_{G},
$$

for then we can use Theorem [6, Theorem 12.1.1] (or Theorem 1.1 from the first part of this work) to find polynomials $Q_{n}\left(x_{1}, \ldots, x_{d-1}, u\right)$ with

$$
\left\|F-Q_{n}\right\|_{[0,1]^{d}} \leq C \omega^{r}(f, 1 / n)_{G},
$$

and then the substitution $u=\Phi\left(x_{1}, \ldots, x_{d}\right)$ will lead to

$$
\left\|f-\tilde{Q}_{n}\right\|_{G_{0}} \leq C \omega^{r}(f, 1 / n)_{G}
$$

for the polynomial $\tilde{Q}_{n}\left(x_{1}, \ldots, x_{d}\right)=Q\left(x_{1}, \ldots, x_{d-1}, \Phi\left(x_{1}, \ldots, x_{d}\right)\right)$.

Thus, everything boils down to proving

$$
\omega^{r}(F, 1 / n)_{\underline{e}_{i}} \leq C \omega^{r}(f, 1 / n)_{G}
$$

for each $i=1,2, \ldots, d$. For $i=d$ this was done in Section 9, see Remark 9.2, so here we shall only consider $i=1,2, \ldots, d-1$ (note that because of the assumed positioning of $G_{0}$, the directions $\underline{e}_{1}, \ldots, \underline{e}_{d-1}$ have a different role with respect to $G_{0}$ than the direction $\underline{e}_{d}$ ), and since these cases are completely similar to each other, we shall assume $i=1$. Thus, let $\zeta_{2}, \ldots, \zeta_{d-1}, \zeta \in[0,1]$ be fixed, and consider the segment $V=\left\{\left(x_{1}, \zeta_{2}, \ldots, \zeta_{d-1}, \zeta\right) \mid x_{1} \in[0,1]\right\}$. We need to estimate the $r$-th difference of $F$ on that segment. As we have seen in Section 8, under the mapping $Y \rightarrow X$ the segment $V$ is mapped into the intersection of $G_{0}$ with the polynomial curve

$$
\gamma_{\zeta_{2}, \ldots, \zeta_{d-1}, \zeta}:=\left\{\left(x_{1}, \zeta_{2}, \ldots, \zeta_{d-1}, x_{d}\right) \mid \Phi\left(x_{1}, \zeta_{2}, \ldots, \zeta_{d-1}, x_{d}\right)=\zeta\right\}
$$

lying in the plane $\left\{x_{2}=\zeta_{2}, \ldots, x_{d-1}=\zeta_{d-1}\right\}$, and the mapping is given by $\left(x, \zeta_{2}, \ldots, \zeta_{d-1}, \zeta\right) \rightarrow\left(x, \zeta_{1}, \ldots, \zeta_{d-1}, \chi_{x, \zeta_{1}, \ldots, \zeta_{d-1}}(\zeta)\right)$ with a real analytic function $\chi_{x, \zeta_{1}, \ldots, \zeta_{d-1}}(\zeta)$. If we write $\sigma(t)$ for $\chi_{t, \zeta_{1}, \ldots, \zeta_{d-1}}(\zeta)$, then we need to estimate the $r$-th difference $\Delta_{h}^{r}$ of the composed function

$$
f\left(t, \zeta_{1}, \ldots, \zeta_{d-1}, \sigma(t)\right) \text {. }
$$

We shall show that this can be done using the modulus of smoothness of $f$ in the intersection of $G_{0}$ with the plane $\left\{x_{2}=\zeta_{2}, \ldots, x_{d-1}=\zeta_{d-1}\right\}$, which is clearly smaller than the modulus of smoothness of $f$ on the whole $G$. Thus, everything is happening in the plane $x_{2}=\zeta_{2}, \ldots, x_{d-1}=\zeta_{d-1}$, so we may suppress the fixed coordinates $\zeta_{2}, \ldots, \zeta_{d-1}$ and we may assume that $d=2$, and $G$ is a domain on the plane. Let us also write $\gamma=\gamma_{\zeta}$ for the curve (11.2) in this case. This is the same as the curve $\{(t, \sigma(t)) \mid t \in[0,1]\}$.

The information on the $r$-th modulus of smoothness on $f$ is given on circles and straight segments, and we need to bound

$$
\sup _{h \leq 1 / n} \sup _{x \in[0,1]} \Delta_{h}^{r} f(x, \sigma(x))
$$


To be more precise, we should bound

$$
\sup _{h \leq 1 / n} \sup _{x \in[0,1]} \Delta_{h \sqrt{x(1-x)}}^{r} f(x, \sigma(x)),
$$

but that is at most as large as the expression in (11.3), so it is sufficient to deal with (11.3). Thus, we need to prove that

$$
\Delta_{h}^{r} f(x, \sigma(x))=\sum_{j=0}^{r}(-1)^{j}\left(\begin{array}{l}
r \\
j
\end{array}\right) f(x+(r / 2-j) h, \sigma(x+(r / 2-j) h))
$$

are $\prec \omega^{r}(f, 1 / n)_{G}$ for $0 \leq h \leq 1 / n$ and $x \in[0,1]$. Here the points $U_{j}=$ $(x+(r / 2-j) h, \sigma(x+(r / 2-j) h)), j=0, \ldots, r$, lie on the curve $\gamma$ and only their $x$-coordinates are equidistant. Our strategy will be the following. First of all, we shall use some polynomial approximant $P_{n}$ to $f$ on some large disk $D$ that contains the points $U_{j}$ which satisfies $\left\|f-P_{n}\right\|_{D} \prec \omega^{r}(f, 1 / n)_{G}$. Then it is enough to consider $\Delta_{h}^{r} P_{n}(x, \sigma(x))$, which can be bounded by $h^{r}$ times an estimate on the $r$-th derivative of $P_{n}$ on the smallest $\operatorname{arc}$ of $\gamma$ that contains all the points $U_{j}$. Thus, we have to find a bound for the $r$-th derivative of the composed function $P_{n}(x, \sigma(x))$, which was done in Section 10.

Thus, we have to estimate $\Delta_{h}^{r} F(x, \zeta)=\Delta_{h}^{r}\left(f\left(x, \gamma_{\zeta}(x)\right)\right.$ for $0 \leq h \leq 1 / n$ with a fixed $\zeta \in[0,1]$. For simplicity we do the estimate for $h=1 / n$ (the $h<1 / n$ case can be similarly handled). Recall that $\gamma=\gamma_{\zeta}$ with $\zeta \in[0,1]$, and first assume that $\zeta \geq L / n^{2}$ for some fixed, but large $L$. Let $x \in[0,1]$ be such that the points $x+(r / 2-j) / n$ belong to $[0,1]$ for $j=0, \ldots, r$. These points correspond to the points $U_{j}=(x+(r / 2-j) / n, \sigma(x+(r / 2-s) / n)), j=0, \ldots, r$, on $\gamma$, which are of distance $\geq c L / n^{2}$ from the "lower" boundary of $G$, which is just the curve $\left(t, \gamma_{0}(t)\right)$. Recall now the $r_{0}$ from the definition of the domain $G$ from the beginning of Section 6.2 , let $C_{r_{0}}$ be the circle of radius $r_{0}$ that lies inside $G$ together with its interior and touches the boundary curve $\gamma_{0}$ at the point $\left(x, \gamma_{0}(x)\right)$, and let $D_{r_{0}}$ be the closed disk enclosed by $C_{r_{0}}$. It is easy to see (see Figure 10) that if $L$ is sufficiently large, then (for large $n$ ) the points $U_{j}$ belong to $D_{r_{0}}$ (recall also that $G_{0}$ was a small part of $G$ ). The points $U_{j}$ lie on the part $\{(u, \gamma(u)) \mid x-r / 2 n \leq u \leq x+r / 2 n\}$ of $\gamma$, and there is a $\rho>0$ depending only on $r_{0}$ and $\Phi$ such that to every point $(u, \gamma(u))$ of that portion of $\gamma$ we can draw a disk of radius $\rho$ that lies in $D_{r_{0}}$ and touches $\gamma$ at the given point.

According to what has been shown in Section 9, for $n=1,2, \ldots$ there are polynomials $P_{n}\left(x_{1}, x_{2}\right)$ of degree at most $n$ such that they approximate $f$ on $D_{r_{0}}$ with error $\leq C \omega^{r}(f, 1 / n)_{G}$. Then, for fixed $\zeta \in[0,1]$,

$$
\left|\Delta_{1 / n}^{r}(F(x, \zeta))\right| \leq\left|\Delta_{1 / n}^{r}\left(F_{n}(x, \zeta)\right)\right|+2^{r} C \omega^{r}(f, 1 / n)_{G},
$$

where $F_{n}(Y)=P_{n}(X)$ (i.e. $F_{n}(x, \zeta)=P_{n}\left(x, \gamma_{\zeta}(x)\right)$ ). The first term on the right is bounded by (see [5, Chap. 2, (7.12)])

$$
\frac{1}{n^{r}} \max _{x-r / 2 n \leq u \leq x+r / 2 n}\left|\frac{\partial^{r} F_{n}}{\partial u^{r}}(u, \zeta)\right|,
$$




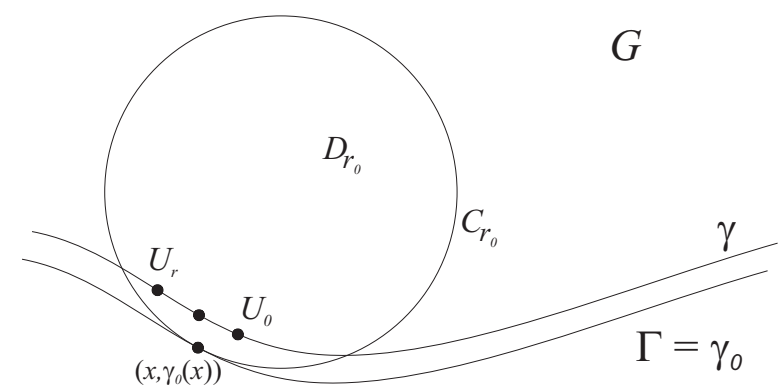

Figure 10: The points $U_{0}, \ldots, U_{r}$ on the curve $\gamma=\gamma_{\zeta_{2}, \ldots, \zeta_{d-1}, \zeta}$

so it is sufficient to show that for each $u$ in the specified range

$$
\left|\frac{\partial^{r} F_{n}}{\partial u^{r}}(u, \zeta)\right| \leq C n^{r} \omega^{r}(f, 1 / n)_{G} .
$$

But on the left we have with $\sigma(t)=\gamma_{\zeta}(t)$

$$
\left|\frac{d^{r} P(t, \sigma(t))}{d t^{r}}\right|_{t=u} \mid
$$

and at the end of Section 10 in (10.9) it was shown that this is $\prec n^{r} \omega(f, 1 / n)$ (set in (10.9) $t_{0}=u$ ). Furthermore, here the $\prec$ depends only on the radius $\rho$ and on the radii $\rho / 2 \leq R_{1}<R_{2}<\cdots<R_{r} \leq \rho$ used in the proof of (10.8), and it is easy to see that these can be selected independently of $x-r / 2 n \leq u \leq x+r / 2 n$, $0 \leq x \leq 1$, if $L$ is sufficiently large (recall that now we are dealing with the situation $\left.\zeta \geq L / n^{2}\right)$. Therefore, we can conclude that this $\prec$ is independent of $u \in[x-r / 2 n, x+r / 2 n], x, x \pm r / 2 n \in[0,1]$. Thus, we have

$$
\left|\frac{d^{r} P_{n}\left(u, \gamma_{\zeta}(u)\right)}{d u^{r}}\right| \prec n^{r} \omega(1 / n),
$$

which is the same as (11.4). This completes the proof of the estimate (11.1) for $i=1$.

In this reasoning we have assumed $\zeta \geq L / n^{2}$. Now we show that the required estimate for $\Delta_{1 / n}^{r} F(x, \zeta)$ in the missing range follows from what we have proven before. Indeed, for a $\zeta \in\left[0, L / n^{2}\right]$, with some large but fixed $\Lambda$, let us choose $w$ so that $w-r \Lambda \sqrt{w(1-w)} / 2 n=\zeta$. Then $\zeta$ is the smallest point in the set $\left\{w_{s}\right\}_{s=0}^{r}, w_{s}:=w+(r / 2-s) \Lambda \sqrt{w(1-w)} / n$, (we have $\zeta=w_{r}$ ), and if $\Lambda$ is sufficiently large, then all these $w_{s}$, except $\zeta$ itself, are bigger than $L / n^{2}$ (note that $\left.w \sim 1 / n^{2}\right)$. Now for each $x+(r / 2-j h) / n, 0 \leq j \leq r$, consider the points $\left(x+(r / 2-j) / n, w_{s}\right), s=0, \ldots, r$, on the vertical segment $I_{x+(r / 2-j) / n}$. By definition,

$$
\left|\sum_{s=0}^{r}(-1)^{s}\left(\begin{array}{l}
r \\
s
\end{array}\right) F\left(x+(r / 2-j) / n, w_{s}\right)\right| \prec \omega^{r}(F, \Lambda / n)_{\underline{e}_{2}},
$$


and above we have already seen that the right hand side is $\prec \omega^{r}(f, 1 / n)_{G}$ (see (9.8)). Furthermore, for each $s<r$

$$
\left|\sum_{j=0}^{r}(-1)^{j}\left(\begin{array}{l}
r \\
j
\end{array}\right) F\left(x+(r / 2-j) / n, w_{s}\right)\right|
$$

is the $\Delta_{1 / n}^{r} F\left(x, w_{s}\right)$ that we have just estimated (for $s<r$ we have $w_{s}>L / n^{2}$ ), so it is $\prec \omega^{r}(f, 1 / n)$. But $\Delta_{1 / n}^{r} F(x, \zeta)$ can be expressed by these:

$$
\begin{aligned}
(-1)^{r} \Delta_{1 / n}^{r} F(x, \zeta) & =(-1)^{r} \sum_{j=0}^{r}(-1)^{j}\left(\begin{array}{l}
r \\
j
\end{array}\right) F(x+(r / 2-j) / n, \zeta) \\
& =\sum_{j=0}^{r}(-1)^{j}\left(\begin{array}{l}
r \\
j
\end{array}\right) \sum_{s=0}^{r}(-1)^{s}\left(\begin{array}{l}
r \\
s
\end{array}\right) F\left(x+(r / 2-j) / n, w_{s}\right) \\
& -\sum_{s=0}^{r-1}(-1)^{s}\left(\begin{array}{l}
r \\
s
\end{array}\right) \sum_{j=0}^{r}(-1)^{j}\left(\begin{array}{l}
r \\
j
\end{array}\right) F\left(x+(r / 2-j) / n, w_{s}\right),
\end{aligned}
$$

so we have a bound $\prec n^{r} \omega(1 / n)$ for it, as well. With this the proof of (11.1), and together with it also the proof of Theorem 6.5 , is complete.

Note added before final production. Feng Dai and Andriy Prymak have recently prepared the manuscript [2] on polynomial approximation on $C^{2}$-domains. In it they defined a new type of modulus of smoothness with which they proved Jackson and converse theorems (exactly as Theorems 6.5 and 6.6) for polynomial approximation on $C^{2}$ domains in any dimension. Their definitions and results are valid for $L^{p}$ spaces, as well. Their modulus is close in spirit to the average moduli of Ivanov [8] mentioned in Remark 6.1,4, and in particular Dai and Prymak were able to deduce both the direct and the converse theorems on any $C^{2}$ domains that were announced in [8]. The main method of [2] is to use Whitney-type local approximation by polynomials of the fixed degree $(r-1) d$ (when one works with $r$-th order of smoothness in $\mathbf{R}^{d}$ ) in conjunction with polynomial partitions of unity similar to the one in the paper [7]. The results and methods in the second part of the present paper allows one to get similar quasi-Whitney local approximants (of fixed degree $\leq 2(r-1) d$ ) involving the moduli of smoothness of the present paper, and from there the procedure used in [2] gives Theorem 6.5 for all $C^{2}$ domains, not just for algebraic ones.

The author is grateful to Zeev Ditzian and Andriy Prymak for valuable and stimulating discussions on the topic of this work. 


\section{References}

[1] B. Bojanov, Elementary proof of the Remez inequality, The American Mathematical Monthly, 100(1993), 483-485.

[2] F. Dai and A. Prymak, Polynomial approximation on $C^{2}$-domains, arXiv:1910.11719

[3] F. Dai and Y. Xu, Moduli of smoothness and approximation on the unit sphere and the unit ball, Adv. Math., 224(2010), 1233-1310.

[4] F. Dai and Y. Xu, Approximation theory and harmonic analysis on spheres and balls. Springer Monographs in Mathematics, Springer, New York, 2013.

[5] R. A. DeVore and G.G. Lorentz, Constructive approximation, Grundlehren der mathematischen Wissenschaften, 303, Springer-Verlag, Berlin, 1993.

[6] Z. Ditzian and V. Totik, Moduli of smoothness, Springer Series for Computational Mathematics, 9, Springer Verlag, New York 1987.

[7] V. K. Dzjadyk and V. N. Konovalov, A method of partition of unity in domains with piecewise smooth boundary into a sum of algebraic polynomials of two variables that have certain kernel properties, Ukrain. Mat. Z., 25(1973), 179-192, 285 (Russian).

[8] K. G. Ivanov, Approximation of functions of two variables by algebraic polynomials. I, Anniversary volume on approximation theory and functional analysis (Oberwolfach, 1983), Internat. Schriftenreihe Numer. Math., vol. 65, Birkhäuser, Basel, 1984, pp. 249-255.

[9] V. N. Konovalov, On some constructive characteristics of some classes of functions of several variables. Dissertation, Kiev, 1972. (Russian)

[10] S. G. Krantz and H. R. Parks, A primer of real analytic functions, Second edition. Birkhäuser Advanced Texts: Basler Lehrbücher. Birkhäuser Boston, Inc., Boston, MA, 2002.

[11] E. J. Remez, Sur une propriété des polynômes de Tchebyscheff, Comm. Inst. Sci. Kharkow. 13(1936), 93-95.

[12] E.B. Saff and V. Totik, Logarithmic Potentials with External Fields, Grundlehren der mathematischen Wissenschaften, 316, Springer Verlag, Berlin, Heidelberg, 1997.

[13] S. B. Stechkin, Generalization of some inequalities of S. N. Bernstein, Dokl. Akad. Nauk., 60(1948), 1511-1514.

[14] V. Totik, Polynomial Approximation on Polytopes, Memoirs of the American Mathematical Society, 212(2014), American Mathematical Society, Providence, Rhode Island, 2014. 
MTA-SZTE Analysis and Stochastics Research Group

Bolyai Institute, University of Szeged

Szeged, Aradi v. tere 1, 6720, Hungary

and

Department of Mathematics and Statistics, University of South Florida 4202 E. Fowler Ave, CMC342, Tampa, FL 33620-5700, USA

totik@mail.usf.edu 